\title{
Alexander invariants and cohomology jump loci in group extensions
}

\author{
Alexander I. Suciu ( $\nabla$ a.suciu@northeastern.edu ) \\ Northeastern University https://orcid.org/0000-0002-5060-7754
}

\section{Research Article}

Keywords: Derived series, rational derived series, mod-\$p\$ derived series, Alexander invariant, rational Alexander invariant, associated graded Lie algebra, Chen Lie algebra, holonomy Lie algebra, infinitesimal Alexander invariant, characteristic variety, resonance variety, formality, group extension

Posted Date: September 17th, 2021

DOl: https://doi.org/10.21203/rs.3.rs-912019/v1

License: (1) This work is licensed under a Creative Commons Attribution 4.0 International License. Read Full License 


\title{
ALEXANDER INVARIANTS AND COHOMOLOGY JUMP LOCI IN GROUP EXTENSIONS
}

\author{
ALEXANDER I. SUCIU ${ }^{1}$
}

\begin{abstract}
Aвstract. We study the integral, rational, and modular Alexander invariants, as well as the cohomology jump loci of groups arising as extensions with trivial algebraic monodromy. Our focus is on extensions of the form $1 \rightarrow K \rightarrow G \rightarrow Q \rightarrow 1$, where $Q$ is an abelian group acting trivially on $H_{1}(K ; \mathbb{Z})$, with suitable modifications in the rational and $\bmod -p$ settings. We find a tight relationship between the Alexander invariants, the characteristic varieties, and the resonance varieties of the groups $K$ and $G$. This leads to an inequality between the respective Chen ranks, which becomes an equality in degrees greater than 1 for split extensions.
\end{abstract}

\section{InTRODUCTION}

1.1. Overview. The Alexander invariants and the characteristic varieties of spaces and groups have their origin in the study of the Alexander polynomials of knots and links. The basic topological idea in defining these invariants is to take the homology of the maximal abelian cover of a CW-complex $X$ and view it as a module over the group ring of $H_{1}(X ; \mathbb{Z})$. One then studies the support loci of these modules, or, alternatively, the characteristic varieties, which are the jump loci for homology with coefficients in rank 1 local systems on $X$. From a group-theoretical point of view, one looks at the derived series of the fundamental group, $G=\pi_{1}(X)$, and views the quotient $B(G)=G^{\prime} / G^{\prime \prime}$ as a module over $\mathbb{Z}\left[G_{\mathrm{ab}}\right]$, filtered by the powers of the augmentation ideal. As noted by Massey in [41], when $G_{\mathrm{ab}}$ is finitely generated, the ranks of the associated graded module of $B(G)$ determine the ranks of the associated graded Lie algebra of $G / G^{\prime \prime}$, also known as the Chen ranks of $G$.

The cohomological version of this theory starts from the low-degrees cup-product map, $\cup_{G}: H^{1}(G) \wedge H^{1}(G) \rightarrow H^{2}(G)$, and builds from it the infinitesimal Alexander invariant $\mathfrak{B}(G)$ and the resonance varieties of $G$. As shown in [25], under a formality

Date: July 11, 2021.

2020 Mathematics Subject Classification. Primary 20F14, 55N25. Secondary 14M12, 16W70, 17B70, 20F40, 20J05, 57M05, 57M07.

Key words and phrases. Derived series, rational derived series, mod- $p$ derived series, Alexander invariant, rational Alexander invariant, associated graded Lie algebra, Chen Lie algebra, holonomy Lie algebra, infinitesimal Alexander invariant, characteristic variety, resonance variety, formality, group extension.

${ }^{1}$ Supported in part by Simons Foundation Collaboration Grants for Mathematicians \#354156 and \#693825. 
assumption, there is a strong relationship between the modules $B(G)$ and $\mathfrak{B}(G)$ on the one hand, and the characteristic and resonance varieties, on the other hand.

We revisit here these classical topics from three main directions. First, we broaden the study of the Alexander invariants to include their rational and modular versions, based on the maximal torsion-free abelian cover and the mod- $p$ congruence covers of a space, or, alternatively, on the rational and mod- $p$ variants of the derived series. Second, we analyze the behavior of the Alexander-type invariants in short exact sequences of the form $1 \rightarrow K \rightarrow G \rightarrow Q \rightarrow 1$, where the quotient group $Q$ is abelian and the sequence stays exact upon abelianization, with suitable modifications in the rational and modular settings. Thirdly, we establish a tight relationship between the cohomology jump loci and the Chen ranks of the groups $G$ and $K$ in such sequences. We proceed now to give a more detailed account of the main results of this paper.

1.2. Lower central series and derived series. Among all the descending series of subgroups associated to a group $G$, the most prominent are the lower central series, $\left\{\gamma_{n}(G)\right\}_{n \geqslant 1}$, and the derived series, $\left\{G^{(r)}\right\}_{r \geqslant 0}$. Following [59, 60, 4, 32, 12, 13, 14, 15], we also consider the rational and mod- $p$ (where $p$ is a prime) versions of these series. All these series start at $G$, and obey the following recursion formulas:

$$
\begin{aligned}
\gamma_{n+1}(G) & =\left[G, \gamma_{n}(G)\right] & G^{(r)} & =\left[G^{(r-1)}, G^{(r-1)}\right], \\
\gamma_{n+1}^{Q}(G) & =\sqrt{\left[G, \gamma_{n}^{\mathrm{Q}}(G)\right]} & G_{\mathrm{Q}}^{(r)} & =\sqrt{\left[G_{\mathrm{Q}}^{(r-1)}, G_{\mathrm{Q}}^{(r-1)}\right]}, \\
\gamma_{n+1}^{p}(G) & =\left(\gamma_{n}^{p}(G)\right)^{p}\left[G, \gamma_{n}^{p}(G)\right] & G_{p}^{(r)} & =\left(G_{p}^{(r-1)}\right)^{p}\left[G_{p}^{(r-1)}, G_{p}^{(r-1)}\right] .
\end{aligned}
$$

Here, for a subset $S \subseteq G$, we let $\sqrt{S}$ denote the set of elements $g \in G$ such that $g^{m} \in S$ for some $m>0$, and we let $S^{p}$ denote the subgroup generated by the set $\left\{g^{p} \mid\right.$ $g \in S\}$, while in (3) the notation $H K$ is shorthand for the subgroup of $G$ generated by $H$ and $K$. The successive quotients of these series are abelian groups, which are torsionfree in case (2) and elementary $p$-groups in case (3). In particular, $G / G^{\prime}=G_{\mathrm{ab}}$ is the abelianization of $G$, while $G / G_{\odot}^{\prime}=G_{\text {abf }}$ is its maximal torsion-free abelian quotient and $G / G_{p}^{\prime}=H_{1}\left(G ; \mathbb{Z}_{p}\right)$ is the maximal elementary $p$-abelian quotient of $G$.

The direct sum of the lower central series quotients, $\operatorname{gr}(G)=\bigoplus_{n \geqslant 1} \gamma_{n}(G) / \gamma_{n+1}(G)$, acquires the structure of a graded Lie algebra, with addition induced from the group multiplication and Lie bracket induced from the group commutator. The associated graded Lie algebra $\operatorname{gr}(G)$ is generated by its degree 1 piece, $\operatorname{gr}_{1}(G)=G_{\mathrm{ab}}$. Thus, if the first Betti number $b_{1}(G)=\operatorname{dim} H_{1}(G, \mathbb{Q})$ is finite, the LCS ranks $\phi_{n}(G)=\operatorname{dim}_{\mathbb{Q}} \operatorname{gr}_{n}(G) \otimes \mathbb{Q}$ are also finite. The graded Lie algebras $\operatorname{gr}^{\complement}(G)$ and $\operatorname{gr}^{p}(G)$ and their graded ranks are defined in like manner. Moreover, if $b_{1}(G)<\infty$, then $\phi_{n}^{\complement}(G)=\phi_{n}(G)$, and if $b_{1}^{p}(G)=\operatorname{dim} H_{1}\left(G, \mathbb{Z}_{p}\right)$ is finite, then so are the mod- $p$ LCS ranks, $\phi_{n}^{p}(G)$.

Replacing in this construction the group $G$ by its maximal metabelian quotient, $G / G^{\prime \prime}$, leads to the Chen Lie algebra, $\operatorname{gr}\left(G / G^{\prime \prime}\right)$, studied in $[9,41,40,45,70]$. We also consider 
the Lie algebras $\operatorname{gr}^{\mathrm{Q}}\left(G / G_{\mathrm{Q}}^{\prime \prime}\right)$ and $\operatorname{gr}^{p}\left(G / G_{p}^{\prime \prime}\right)$. The graded ranks of all these Lie algebras, $\theta_{n}(G)=\theta_{n}^{\mathrm{Q}}(G)$ and $\theta_{n}^{p}(G)$, are bounded above by the corresponding LCS ranks.

1.3. Alexander invariants. In what follows, we are primarily interested in the Alexander-type invariants associated with the three kinds of derived series mentioned above. The classical Alexander invariant of $G$ is the abelian group $B(G)=G^{\prime} / G^{\prime \prime}$, viewed as a module over the group-ring $\mathbb{Z}\left[G_{\mathrm{ab}}\right]$. We introduce and study here two variations thereof: the rational Alexander invariant is the quotient $B_{\mathrm{Q}}(G)=G_{\mathrm{Q}}^{\prime} / G_{\mathrm{Q}}^{\prime \prime}$, viewed as a module over $\mathbb{Z}\left[G_{\mathrm{abf}}\right]$, while the mod-p Alexander invariant is the quotient $B_{p}(G)=G_{p}^{\prime} / G_{p}^{\prime \prime}$, viewed as a module over $\mathbb{Z}_{p}\left[H_{1}\left(G ; \mathbb{Z}_{p}\right)\right]$. In the process, we define analogues of the Crowell exact sequence, relating the the Alexander invariant, the Alexander module, and the augmentation ideal of the group ring. Important to our analysis are the naturality properties of these constructions. For instance, every homomorphism $\alpha: G \rightarrow H$ gives rise to a morphism of modules, $B(\alpha): B(G) \rightarrow B(H)$, which covers the ring map $\tilde{\alpha}_{\mathrm{ab}}: \mathbb{Z}\left[G_{\mathrm{ab}}\right] \rightarrow \mathbb{Z}\left[H_{\mathrm{ab}}\right]$. In turn, $B(\alpha)$ factors through a $\mathbb{Z}\left[G_{\mathrm{ab}}\right]$-linear map from $B(G)$ to the module $B(H)_{\alpha}$ obtained from $B(H)$ by restriction of scalars along $\tilde{\alpha}_{\mathrm{ab}}$.

In [41], Massey uncovered a fruitful connection between the Alexander invariant of a group and the lower central series of its maximal metabelian quotient. In $\S 6$ we provide a complete account of this result (part (1) of the theorem below), and establish rational and modular analogues of Massey's correspondence. We summarize our results, as follows.

Theorem 1.1. For a group $G$, the following hold, for all $n \geqslant 0$.

(1) $I^{n} B(G)=\gamma_{n+2}\left(G / G^{\prime \prime}\right)$, where $I$ is the augmentation ideal of $\mathbb{Z}\left[G_{\mathrm{ab}}\right]$.

(2) $I^{n} \cdot\left(B_{\mathbb{Q}}(G) \otimes \mathbb{Q}\right)=\gamma_{n+2}^{\mathbb{Q}}\left(G / G_{\mathbb{Q}}^{\prime \prime}\right) \otimes \mathbb{Q}$, where I is the augmentation ideal of $\mathbb{Q}\left[G_{\mathrm{abf}}\right]$.

(3) $I^{n} B_{p}(G)=\gamma_{n+2}\left(G / G_{p}^{\prime \prime}\right)$, where $I$ is the augmentation ideal of $\mathbb{Z}_{p}\left[H_{1}\left(G ; \mathbb{Z}_{p}\right)\right]$.

When $G$ is finitely generated, this theorem allows us to express the generating series for the three flavors of Chen ranks in terms of the Hilbert series for the corresponding Alexander invariants. We also relate the integral and rational versions of the Alexander invariant. In Proposition 3.5 we show that the inclusion $G^{\prime} \hookrightarrow G_{\mathbb{Q}}^{\prime}$ induces a functorial morphism, $\kappa: B(G) \rightarrow B_{\mathbb{Q}}(G)$. When Tors $\left(G_{\mathrm{ab}}\right)$ is finite, the map $\kappa \otimes \mathbb{Q}: B(G) \otimes \mathbb{Q} \rightarrow$ $B_{\mathbb{Q}}(G) \otimes \mathbb{Q}$ is surjective, but in general it is not an isomorphism. Nevertheless, if $b_{1}(G)<$ $\infty$, we prove in Theorem 6.9 , that $\kappa \otimes \mathbb{Q}$ induces isomorphisms on $I$-adic completions and associated graded modules. This theorem partly overlaps with a result from [20], where the group $G_{\mathrm{Q}}^{\prime}$ is called the Johnson kernel of $G$, due to the role it plays in D. Johnson's study of the Torelli group of a surface and of the Johnson homomorphism.

1.4. Group extensions. Our main focus in this paper is on how the algebraic and geometric invariants of groups mentioned above behave under group extensions. Given a short exact sequence of groups,

$$
1 \rightarrow K \stackrel{\iota}{\rightarrow} G \stackrel{\pi}{\rightarrow} Q \rightarrow 1,
$$


we relate-under suitable assumptions - the Alexander invariants, the characteristic varieties, the associated graded Lie algebras, and the Chen Lie algebras of the group $G$ to those of its subgroup $K$. The assumptions we make are tailored to the three versions (integral, rational, and modular) under consideration, and are basically of two types.

One type of constraint is on the group $Q$ : we require it to be abelian, specializing to torsion-free in the $\mathbb{Q}$-version and elementary $p$-abelian in the mod- $p$ version. The other type of constraint in on the exactness of the sequence obtained from the given one by applying the functors sending $G$ to $G_{\mathrm{ab}}, G_{\mathrm{abf}}$, and $G_{\mathrm{ab}} \otimes \mathbb{Z}_{p}$, respectively; when that happens, we say (4) is an ab-exact, abf-exact, or $p$-exact sequence, respectively. When the given sequence splits, these conditions amount to the triviality of the action of $Q$ on $K_{\mathrm{ab}}, K_{\mathrm{abf}}$ (or $K_{\mathrm{ab}} \otimes \mathbb{Q}$ if $K_{\mathrm{abf}}$ is finitely generated), and $K_{\mathrm{ab}} \otimes \mathbb{Z}_{p}$, respectively. In the split case, our study relies in good measure on work of $[29,54,5,30]$ and our recent results from [66] regarding the several types of lower central series and associated graded Lie algebras of split extensions.

1.5. Alexander invariants in group extensions. We are now in a position to summarize our main results connecting the aforementioned algebraic invariants of a group $G$ and a normal subgroup $K \triangleleft G$. The first result deals with the integral case, and is proved in Theorem 8.7 and Corollary 8.8, where more detailed statements can be found.

Theorem 1.2. Suppose $1 \rightarrow K \stackrel{\iota}{\rightarrow} G \rightarrow Q \rightarrow 1$ is an ab-exact sequence of groups, and $Q$ is abelian. Then,

(1) The induced map on Alexander invariants, $B(\iota): B(K) \rightarrow B(G)$, factors through $a \mathbb{Z}\left[K_{\mathrm{ab}}\right]$-linear isomorphism, $B(K) \rightarrow B(G)_{\iota}$.

(2) If $G_{\mathrm{ab}}$ is finitely generated, then $\theta_{n}(K) \leqslant \theta_{n}(G)$ for all $n \geqslant 1$.

(3) If the sequence is split exact, then $\iota$ induces isomorphisms of graded Lie algebras, $\operatorname{gr}_{\geqslant 2}(K) \stackrel{\simeq}{\longrightarrow} \operatorname{gr}_{\geqslant 2}(G)$ and $\operatorname{gr}_{\geqslant 2}\left(K / K^{\prime \prime}\right) \stackrel{\simeq}{\longrightarrow} \operatorname{gr}_{\geqslant 2}\left(G / G^{\prime \prime}\right)$. Consequently, if $b_{1}(G)<\infty$, then $\phi_{n}(K)=\phi_{n}(G)$ and $\theta_{n}(K)=\theta_{n}(G)$ for all $n \geqslant 2$.

When $G=G_{\Gamma}$ is the right-angled Artin group associated to a finite simple graph $\Gamma$ and $K=N_{\Gamma}$ is the corresponding Bestvina-Brady group from [7], the above theorem recovers in a slightly stronger form several results from [47]. The next result deals with the rational case, and is proved in Theorem 9.8 and Corollary 9.9.

Theorem 1.3. Suppose (4) is an abf-exact sequence and $Q$ is torsion-free abelian. Then,

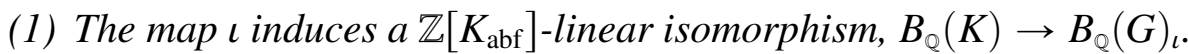

(2) If $G_{\text {abf }}$ is finitely generated, then $\theta_{n}(K) \leqslant \theta_{n}(G)$ for all $n \geqslant 1$.

(3) If the sequence is split exact, then $\iota$ induces isomorphisms of graded Lie algebras, $\operatorname{gr}_{\geqslant 2}^{\mathrm{e}}(K) \stackrel{\simeq}{\longrightarrow} \mathrm{gr}_{\geqslant 2}^{\mathrm{e}}(G)$ and $\mathrm{gr}_{\geqslant 2}^{\mathrm{e}}\left(K / K^{\prime \prime}\right) \stackrel{\simeq}{\longrightarrow} \mathrm{gr}_{\geqslant 2}^{\mathrm{e}}\left(G / G^{\prime \prime}\right)$. Consequently, if $b_{1}(G)<\infty$, then $\phi_{n}(K)=\phi_{n}(G)$ and $\theta_{n}(K)=\theta_{n}(G)$ for all $n \geqslant 2$. 
One instance where this theorem applies is the case when $K$ is the fundamental group of a connected CW-complex $X$ with $b_{1}(X)<\infty$ and $G$ is the fundamental group of the mapping torus of a map $f: X \rightarrow X$ inducing the identity on $H_{1}(X ; \mathbb{Q})$.

The last result of this type deals with the modular case, and is proved in Theorem 10.4 and Corollary 10.5 .

Theorem 1.4. Suppose (4) is a p-exact sequence and $Q$ is an elementary abelian $p$ group. Then

(1) The map ı induces a $\mathbb{Z}_{p}\left[K_{\mathrm{ab}} \otimes \mathbb{Z}_{p}\right]$-linear isomorphism, $B_{p}(K) \rightarrow B_{p}(G)$.

(2) If $b_{1}^{p}(G)<\infty$, then $\theta_{n}^{p}(K) \leqslant \theta_{n}^{p}(G)$ for all $n \geqslant 1$.

(3) If the sequence is split exact, then $\iota$ induces isomorphisms of graded Lie algebras, $\operatorname{gr}_{\geqslant 2}^{p}(K) \stackrel{\simeq}{\longrightarrow} \operatorname{gr}_{\geqslant 2}^{p}(G)$ and $\operatorname{gr}_{\geqslant 2}^{p}\left(K / K^{\prime \prime}\right) \stackrel{\simeq}{\longrightarrow} \operatorname{gr}_{\geqslant 2}^{p}\left(G / G^{\prime \prime}\right)$. Moreover, if $b_{1}^{p}(G)<\infty$, then $\phi_{n}^{p}(K)=\phi_{n}^{p}(G)$ and $\theta_{n}^{p}(K)=\theta_{n}^{p}(G)$ for all $n \geqslant 2$.

1.6. Characteristic varieties. For a finitely generated group $G$, the set of complexvalued characters, $\mathbb{T}_{G}=\operatorname{Hom}\left(G, \mathbb{C}^{*}\right)$, is an abelian complex algebraic group, with coordinate ring $\mathbb{C}\left[G_{\mathrm{ab}}\right]$. The group $\mathbb{T}_{G}$ is isomorphic to $\left(\mathbb{C}^{*}\right)^{r} \times \operatorname{Tors}\left(G_{\mathrm{ab}}\right)$, where $r=$ $\operatorname{rank}\left(G_{\mathrm{ab}}\right)$. This group may be thought of as the moduli space of rank 1 local systems on a connected CW-complex $X$ with fundamental group $G$. Taking homology with coefficients in such local systems carves out subvarieties $\mathscr{V}_{k}(G) \subset \mathbb{T}_{G}$ where the homology in degree 1 has rank at least $k$.

The geometry of these varieties is intimately related to the homological and finiteness properties of normal subgroups $K \triangleleft G$ with abelian quotient $Q=G / K$ and of regular, abelian covers of spaces $X$ with $\pi_{1}(X)=G$. For instance, the stratification of the character group by the varieties $\mathscr{V}_{k}(G)$ determines the first Betti number of any finite abelian cover $Y$ as above, see e.g. [38, 44]. The characteristic varieties also carry precise information regarding the homological and geometric finiteness properties of infinite abelian covers, see e.g. [26, 24, 50, 62], and provide powerful obstructions to formality and quasi-projectivity of spaces and groups, see e.g. [24, 25, 49].

The characteristic varieties of a group are controlled by its Alexander invariant in a manner that is crucial to our analysis. More precisely, $\mathscr{V}_{k}(G)$ coincides (at least away from the identity character 1$)$, with the support of the $\mathbb{C}\left[G_{\mathrm{ab}}\right]$-module $\wedge^{k} B(G) \otimes \mathbb{C}$. Furthermore, letting $\mathscr{W}_{k}(G)$ be the intersection of $\mathscr{V}_{k}(G)$ with the identity component of the character group, we have that $\mathscr{W}_{k}(G)=\operatorname{supp}\left(\wedge^{k} B_{Q}(G) \otimes \mathbb{C}\right)$, at least away from 1. Although results along these lines have been known for a long time (see e.g. [38, 34, 44, 24]), there does not appear to be a complete proof in the literature, at least not in the generality posited here; therefore, we supply full details in Theorems 12.6 and 12.9.

1.7. Characteristic varieties in group extensions. We are now in a position to summarize our main results connecting the characteristic varieties of groups $G$ and $K$ as above.

Theorem 1.5. Let $1 \rightarrow K \stackrel{\iota}{\rightarrow} \rightarrow Q \rightarrow 1$ be an exact sequence of finitely generated groups. 
(1) If the sequence is ab-exact and $Q$ is abelian, then the map $\iota^{*}: \mathbb{T}_{G} \rightarrow \mathbb{T}_{K}$ restricts to maps $\iota^{*}: \mathscr{V}_{k}(G) \rightarrow \mathscr{V}_{k}(K)$ for all $k \geqslant 1$; furthermore, $\iota^{*}: \mathscr{V}_{1}(G) \rightarrow \mathscr{V}_{1}(K)$ is a surjection.

(2) If the sequence is abf-exact and $Q$ is torsion-free abelian, then the map $\iota^{*}: \mathbb{T}_{G}^{0} \rightarrow$ $\mathbb{T}_{K}^{0}$ restricts to maps $\iota^{*}: \mathscr{W}_{k}(G) \rightarrow \mathscr{W}_{k}(K)$ for all $k \geqslant 1 ;$ furthermore, $\iota^{*}: \mathscr{W}_{1}(G) \rightarrow$ $\mathscr{W}_{1}(K)$ is a surjection.

This result is proved in Theorem 13.3. As a corollary, we show the following: If $\mathscr{V}_{1}(G)$ is finite (in the first case), or $\mathscr{W}_{1}(G)$ is finite (in the second case), then the Chen ranks $\theta_{n}(K)$ vanish for all sufficiently large $n$.

In upcoming work [67] we will apply Theorem 1.5 in the case when $G$ is the fundamental group of the complement in $\mathbb{C}^{\ell}$ of an arrangement of linear hyperplanes, $K$ is the group of its Milnor fiber, and the monodromy of the Milnor fibration acts trivially on $H_{1}(K ; \mathbb{Z})$ or $H_{1}(K ; \mathbb{Q})$, respectively. Examples from [64, 67] show that the maps $\iota^{*}: \mathscr{V}_{k}(G) \rightarrow \mathscr{V}_{k}(K)$ and $\iota^{*}: \mathscr{W}_{k}(G) \rightarrow \mathscr{W}_{k}(K)$ may fail to be surjective for $k>1$, even in this very special context. This phenomenon leads to subtle invariants that can distinguish the homotopy types of Milnor fibers of certain arrangements whose complements are homotopy equivalent.

1.8. Holonomy, formality, and resonance. There are two other important Lie algebras associated to a finitely generated group $G$. The first one is the holonomy Lie algebra, $\mathfrak{h}(G)$, which was defined by Chen [10] as the quotient of the free Lie algebra on $G_{\text {abf }}$ by the Lie ideal generated by the image of the dual of the cup-product map $\cup_{G}$. This is a quadratic Lie algebra which maps surjectively to $\operatorname{gr}(G)$. The graded ranks of its second derived quotient, $\bar{\theta}_{n}(G)$ - known as the holonomy Chen ranks-are bounded below by the usual Chen ranks. Following [45], we use the holonomy Lie algebra to construct an infinitesimal version of the Alexander invariant, $\mathfrak{B}(G)=\mathfrak{h}(G)^{\prime} / \mathfrak{h}(G)^{\prime \prime}$, which is a graded module over the symmetric algebra $\operatorname{Sym}\left(G_{\text {abf }}\right)$ whose graded ranks coincide with the holonomy Chen ranks, after a shift of 2.

From a rational homotopy point of view, most important is the Malcev Lie algebra, $\mathfrak{m}(G)$, defined by Quillen in [57] as the (complete, filtered) Lie algebra of primitive elements in the $I$-adic completion of $\mathbb{Q}[G]$. The associated graded Lie algebra with respect to this filtration, $\operatorname{gr}(\mathfrak{m}(G))$, is isomorphic to $\operatorname{gr}(G) \otimes \mathbb{Q}$. The group $G$ is said to be graded formal if the canonical surjection $\mathfrak{h}(G) \otimes \mathbb{Q} \rightarrow \operatorname{gr}(G) \otimes \mathbb{Q}$ is an isomorphism; it is 1 -formal if, in addition, $\mathfrak{m}(G)$ is isomorphic, as a filtered Lie algebra, to the completion of $\operatorname{gr}(G) \otimes \mathbb{Q}$ with respect to the bracket-length filtration.

The resonance varieties of $G$ are infinitesimal analogues of the characteristic varieties, defined purely in terms of cohomological data. More precisely, let $H^{\bullet}=H^{\bullet}(G ; \mathbb{C})$ be the cohomology algebra of $G$. For each $k \geqslant 1$, the depth $k$ resonance variety $\mathscr{R}_{k}(G)$ consists of all elements $a \in H^{1}$ for which there exist $u_{1}, \ldots, u_{k} \in H^{1}$ such that $a u_{i}=0$ in $H^{2}$ and $\left\{a, u_{1}, \ldots, u_{k}\right\}$ are linearly independent. These sets are homogeneous algebraic subvarieties of the affine space $H^{1}=\mathbb{C}^{r}$ which are controlled by the infinitesimal 
Alexander invariant; more exactly, $\mathscr{R}_{k}(G)=\operatorname{supp}\left(\bigwedge^{k} \mathfrak{B}(G) \otimes \mathbb{C}\right)$, at least away from 0 . This was proved in $[23,22]$ in the case when $G$ is finitely generated; we give in Theorem 16.2 a different proof_-valid for all groups with $b_{1}(G)<\infty$-based on the BGG correspondence and an infinitesimal version of the Crowell exact sequence.

The resonance varieties capture deep information about qualitative properties and numerical invariants of a finitely generated group $G$. When compared to the characteristic varieties via the Tangent Cone theorem of [25], they obstruct $G$ from being 1-formal, or being realizable as the fundamental group of a quasi-projective or Kähler manifold. In favorable situations, they do allow for the computation of the Chen ranks $\theta_{n}(G)$ in terms of the dimensions of the irreducible components of $\mathscr{R}_{1}(G)$; see $[61,16,1,3]$. Finally, they have applications to the study of homological finiteness properties in the Johnson filtration of mapping class groups and automorphism groups of free groups, and to Green's conjecture on free resolutions of canonical curves; see [51, 22, 20, 53, 2].

1.9. Resonance varieties in extensions. Our final result relates the resonance varieties of a group $G$ to those of a normal subgroup $K \triangleleft G$, under suitable assumptions on the quotient $Q=G / K$ and its action on the first homology of $K$, as well as on the formality properties of $G$ and $K$. In Theorems 17.2 and 17.6, we establish the following result.

Theorem 1.6. Let $1 \rightarrow K \stackrel{\iota}{\rightarrow} \rightarrow \rightarrow Q \rightarrow 1$ be an exact sequence of finitely generated groups. Suppose that either one of the following set of conditions is satisfied.

(1) The sequence is split exact, $G$ is graded formal, $Q$ is abelian, and $Q$ acts trivially on $H_{1}(K ; \mathbb{Q})$.

(2) The sequence if ab-exact, $G$ and $K$ are 1 -formal, and $Q$ is abelian.

(3) The sequence if abf-exact, $G$ and $K$ are 1-formal, and $Q$ is torsion-free abelian.

Then the homomorphism $\iota^{*}: H^{1}(G, \mathbb{C}) \rightarrow H^{1}(K, \mathbb{C})$ restricts to maps $\iota^{*}: \mathscr{R}_{k}(G) \rightarrow$ $\mathscr{R}_{k}(K)$ for all $k \geqslant 1$; furthermore, the map $\iota^{*}: \mathscr{R}_{1}(G) \rightarrow \mathscr{R}_{1}(K)$ is a surjection.

In particular, if any one of the above conditions is satisfied and $\mathscr{R}_{1}(G) \subseteq 0$, then $\mathscr{R}_{1}(K) \subseteq 0$, and thus the $\mathbb{Q}$-vector space $\mathfrak{B}(K) \otimes \mathbb{Q}$ is finite-dimensional. Under the 1 -formality assumptions from either (2) or (3), we conclude that $\theta_{n}(G)$ and $\theta_{n}(K)$ vanish for sufficiently large $n$.

When $G=G_{\Gamma}$ and $N=N_{\Gamma}$ are the right-angled Artin group and the Bestvina-Brady group associated to a graph $\Gamma$, the theorem recovers a result from [47] and extends it further. In [67] we will apply Theorem 1.6 in the case when $G$ is an arrangement group, $K$ is the group of its Milnor fiber, and the monodromy of the Milnor fibration acts trivially on $H_{1}(K ; \mathbb{Q})$.

1.10. Organization. The paper is organized in four parts of roughly equal length.

Part I deals with the Alexander invariants, the derived series, and the lower central series of a group. We concentrate in $\$ 2$ on the classical derived series and the associated Alexander invariant. In $\S 3$ we turn to the rational version of these objects, while in $\S 4$ we 
study the mod- $p$ version. We conclude in $\$ 5$ with a quick review of the corresponding lower central series, associated graded Lie algebras, and Chen Lie algebras.

Part II explores group extensions of the form $1 \rightarrow K \rightarrow G \rightarrow Q \rightarrow 1$, focussing on the relationship between the Alexander invariants and the lower central series of those groups. We start in $\S 6$ with Massey's correspondence between the filtration of the Alexander invariant of $K$ by powers of the augmentation ideal of $Q$ and the lower central series of the maximal metabelian quotient of $G$. We continue in $\S 7$ with an overview of the lower central series and associated graded algebras of split extensions of groups. In $\S 8$ we explore ab-exact sequences, and establish our results on the way the Alexander invariants and the Chen ranks behave under such extensions. We prove analogous results for abf-exact sequences in $\S 9$ and for $p$-exact sequences in $\S 10$.

Parts III and IV contain a detailed study of the cohomology jump loci of a finitely generated group, and the way these loci behave under the aforementioned kinds of group extensions. We start in $\$ 11$ with the characteristic varieties, and continue in $\S 12$ with the Alexander varieties, focussing on the relationship between the two. In $\S 13$ we establish our structural results relating the characteristic varieties of a group $G$ to those of a normal subgroup $K$ under appropriate assumptions. After discussing in $\$ 14$ the Malcev and holonomy Lie algebras of a group and the resulting notion of 1-formality, we provide in $\$ 15$ and $\$ 16$ detailed information on the infinitesimal Alexander invariant and the resonance varieties. Finally, we establish in $\S 17$ our results on the way resonance behaves in ab- and abf-exact group extensions, under suitable formality hypothesis.

\section{Part I. Alexander invariants}

\section{Derived Series and the Alexander invariant}

We start with a review of the derived series and the Alexander invariant of a group, and discuss some of their basic properties.

2.1. Abelianization and derived series. Let $G$ be a group. If $H$ and $K$ are subgroups of $G$, then $[H, K]$ denotes the subgroup of $G$ generated by all elements of the form $[a, b]=a b a^{-1} b^{-1}$ with $a \in H$ and $b \in K$. If both $H$ and $K$ are normal subgroups, then their commutator $[H, K]$ is again a normal subgroup. Moreover, if $\alpha: G \rightarrow H$ is a homomorphism, then

$$
\alpha([H, K]) \subseteq[\alpha(H), \alpha(K)] .
$$

Taking $H=K=G$ in this construction we obtain the commutator (or, derived) subgroup, $G^{\prime}=[G, G]$. The quotient group, $G_{\mathrm{ab}}:=G / G^{\prime}$ is called the abelianization of $G$, and is characterized by the fact that it is the maximal abelian quotient of $G$. We denote by ab: $G \rightarrow G_{\mathrm{ab}}$ the abelianization homomorphism, whose kernel is $G^{\prime}$. The torsion elements of $G_{\mathrm{ab}}$ form a subgroup; the quotient group, $G_{\mathrm{abf}}:=G_{\mathrm{ab}} / \operatorname{Tors}\left(G_{\mathrm{ab}}\right)$ is called the torsion-free abelianization of $G$, and is characterized by the fact that it is the maximal torsion-free abelian quotient of $G$. 
The derived series of $G$, denoted $\left\{G^{(r)}\right\}_{r \geqslant 0}$, is defined inductively by setting $G^{(0)}=G$ and $G^{(r)}=\left[G^{(r-1)}, G^{(r-1)}\right]$; in particular, $G^{(1)}=G^{\prime}$ and $G^{(2)}=G^{\prime \prime}$. Using (5) and induction on $r$, it is readily seen that the terms of the derived series are fully invariant subgroups; that is, if $\alpha: G \rightarrow H$ is a group homomorphism, then $\alpha\left(G^{(r)}\right) \subseteq H^{(r)}$, for all $r$. Consequently, the derived series is a normal series, i.e., $G^{(r)} \triangleleft G$, for all $r$. Moreover, since $G^{(r-1)} / G^{(r)}=\left(G^{(r-1)}\right)_{\mathrm{ab}}$, all the successive quotients of the series are abelian groups.

A group $G$ is said to be solvable if its derived series of $G$ terminates in finitely many steps; that is, $G^{(\ell)}=\{1\}$ for some integer $\ell \geqslant 0$. The smallest such integer, $\ell(G)$, is then called the derived length of $G$. Clearly, $\ell(G) \leqslant 1$ if and only if $G$ is abelian, while $\ell(G) \leqslant 2$ if and only if $G$ is metabelian. The maximal solvable quotient of $G$ of length $r$ is $G / G^{(r)}$; in particular, the maximal metabelian quotient is $G / G^{\prime \prime}$.

2.2. Alexander invariant and Alexander module. Among the successive quotients of the derives series of a group $G$, the second one plays a special role. The Alexander invariant of $G$ is the abelian group

$$
B(G):=G^{\prime} / G^{\prime \prime},
$$

viewed as a module over the group-ring $\mathbb{Z}\left[G_{\mathrm{ab}}\right]$; alternatively, $B(G)=G_{\mathrm{ab}}^{\prime}=H_{1}\left(G_{\mathrm{ab}}^{\prime} ; \mathbb{Z}\right)$. Addition in $B(G)$ is induced from multiplication in $G$, to wit, $\left(x G^{\prime \prime}\right)+\left(y G^{\prime \prime}\right)=x y G^{\prime \prime}$ for all $x, y \in G^{\prime}$, while scalar multiplication is induced from conjugation in the maximal metabelian quotient, $G / G^{\prime \prime}$, via the exact sequence

$$
1 \longrightarrow G^{\prime} / G^{\prime \prime} \longrightarrow G / G^{\prime \prime} \longrightarrow G / G^{\prime} \longrightarrow 1 \text {. }
$$

That is, $g G^{\prime} \cdot x G^{\prime \prime}=g x g^{-1} G^{\prime \prime}$ for all $g \in G$ and $x \in G^{\prime}$, with the action of $G / G^{\prime}=G_{\mathrm{ab}}$ extended $\mathbb{Z}$-linearly to the whole of $\mathbb{Z}\left[G_{\mathrm{ab}}\right]$.

The augmentation map, $\varepsilon: \mathbb{Z}[G] \rightarrow \mathbb{Z}$, is the linear extension to group rings of the trivial homomorphism, $G \rightarrow\{1\}$; let $I(G)=\operatorname{ker}(\varepsilon)$ be the augmentation ideal. Closely related to the Alexander invariant is the Alexander module of $G$,

$$
A(G)=\mathbb{Z}\left[G_{\mathrm{ab}}\right] \otimes_{\mathbb{Z}[G]} I(G),
$$

with $\mathbb{Z}\left[G_{\mathrm{ab}}\right]$-module structure coming from multiplication on the left factor.

In order to better understand the $\mathbb{Z}\left[G_{\mathrm{ab}}\right]$-modules $A(G)$ and $B(G)$, we will look at them next from a topological point of view, following the approach of Massey from [41].

2.3. Topological interpretation. Let $X$ be a connected CW-complex with $\pi_{1}\left(X, x_{0}\right)=$ $G$. (We may assume $X$ has a single 0 -cell, which we then take as the basepoint $x_{0}$.) Lifting the cell structure of $X$ to the maximal abelian cover, $q: X^{\mathrm{ab}} \rightarrow X$, we obtain an augmented chain complex of free $\mathbb{Z}\left[G_{\mathrm{ab}}\right]$-modules,

$$
\cdots \longrightarrow C_{2}\left(X^{\mathrm{ab}} ; \mathbb{Z}\right) \stackrel{\partial_{2}^{\mathrm{ab}}}{\longrightarrow} C_{1}\left(X^{\mathrm{ab}} ; \mathbb{Z}\right) \stackrel{\partial_{1}^{\mathrm{ab}}}{\longrightarrow} C_{0}\left(X^{\mathrm{ab}} ; \mathbb{Z}\right) \stackrel{\varepsilon}{\longrightarrow} \mathbb{Z} \longrightarrow 0,
$$


where $C_{0}\left(X^{\mathrm{ab}} ; \mathbb{Z}\right)=\mathbb{Z}\left[G_{\mathrm{ab}}\right]$ and $\varepsilon$ is the augmentation map. Since $\pi_{1}\left(X^{\mathrm{ab}}\right)=G^{\prime}$, the Alexander invariant $B(G)=\left(G^{\prime}\right)_{\text {ab }}$ is isomorphic to $H_{1}\left(X^{\mathrm{ab}} ; \mathbb{Z}\right)$, the first homology group of the chain complex (9), with module structure induced by the action of $G_{\mathrm{ab}}$ by deck transformations. In other words, $B(G)=H_{1}\left(X ; \mathbb{Z}\left[G_{\mathrm{ab}}\right]\right)$.

Example 2.1. Let $X=\bigvee^{n} S^{1}$ be a wedge of $n$ circles. Identify $\pi_{1}(X)$ with the free group $F_{n}=\left\langle x_{1}, \ldots, x_{n}\right\rangle$ and $\left(F_{n}\right)_{\mathrm{ab}}$ with $\mathbb{Z}^{n}$. The chain complex $\left(C_{i}\left(\left(T^{n}\right)^{\mathrm{ab}} ; \mathbb{Z}\right), \partial_{i}^{\mathrm{ab}}\right)$ of the universal (abelian) cover of the $n$-torus $T^{n}=K\left(\mathbb{Z}^{n}, 1\right)$ may be viewed as the Koszul complex on elements $t_{1}-1, \ldots, t_{n}-1$ over the ring $\mathbb{Z}\left[\mathbb{Z}^{n}\right]=\mathbb{Z}\left[t_{1}^{ \pm 1}, \ldots, t_{n}^{ \pm 1}\right]$. The Alexander invariant $B\left(F_{n}\right)$, then, equals the $\mathbb{Z}\left[\mathbb{Z}^{n}\right]$-module coker $\left(\partial_{3}^{\text {ab }}\right)$; in particular,

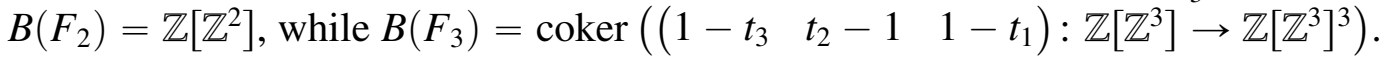

Consider now the fiber $F=q^{-1}\left(x_{0}\right)$, and fix a basepoint $\tilde{x}_{0} \in F$. The homology long exact sequence for the pair $\left(X^{\mathrm{ab}}, F\right)$ yields an exact sequence of $\mathbb{Z}\left[G_{\mathrm{ab}}\right]$-modules,

$$
0 \longrightarrow H_{1}\left(X^{\mathrm{ab}} ; \mathbb{Z}\right) \longrightarrow H_{1}\left(X^{\mathrm{ab}}, F ; \mathbb{Z}\right) \longrightarrow H_{0}(F ; \mathbb{Z}) \longrightarrow H_{0}\left(X^{\mathrm{ab}} ; \mathbb{Z}\right) \longrightarrow 0 \text {. }
$$

As noted previously, the first term is the Alexander invariant $B(G)$. The second term is the Alexander module $A(G)$; indeed, sending an element $g-1 \in I(G)$ to the path in $X^{\mathrm{ab}}$ from $\tilde{x}_{0}$ to $g \tilde{x}_{0}$ obtained by lifting the loop $g$ at $\tilde{x}_{0}$ induces an isomorphism $A(G) \stackrel{\simeq}{\longrightarrow}$ $H_{1}\left(X^{\mathrm{ab}}, F ; \mathbb{Z}\right)$. Finally, the homomorphism $H_{0}(F ; \mathbb{Z}) \rightarrow H_{0}\left(X^{\mathrm{ab}} ; \mathbb{Z}\right)$ may be identified with the augmentation map, $\varepsilon: \mathbb{Z}\left[G_{\mathrm{ab}}\right] \rightarrow \mathbb{Z}$. Therefore,

$$
A(G)=\operatorname{coker}\left(\partial_{2}^{\mathrm{ab}}\right) \text {, }
$$

and the sequence (10) yields an exact sequence of $\mathbb{Z}\left[G_{\mathrm{ab}}\right]$-modules,

$$
0 \longrightarrow B(G) \longrightarrow A(G) \longrightarrow I\left(G_{\mathrm{ab}}\right) \longrightarrow 0,
$$

known as the Crowell exact sequence of the group, cf. [18, 41]. When $G_{\mathrm{ab}}$ is finitely generated, the ring $\mathbb{Z}\left[G_{\mathrm{ab}}\right]$ is Noetherian. In this case, the Alexander module $A(G)$ is finitely generated, and hence the presentation (11) may be reduced to a finite presentation. Thus, by (12), the Alexander invariant $B(G)$ may also be finitely presented.

If $G$ admits a finite presentation, say, $G=\left\langle x_{1}, \ldots, x_{m} \mid r_{1}, \ldots, r_{\ell}\right\rangle$, the $\mathbb{Z}\left[G_{\mathrm{ab}}\right]$-linear map $\partial_{2}^{\mathrm{ab}}: \mathbb{Z}\left[G_{\mathrm{ab}}\right]^{\ell} \rightarrow \mathbb{Z}\left[G_{\mathrm{ab}}\right]^{m}$ from (9) may be identified with the classical Alexander matrix, whose entries are the abelianized Fox derivatives of the relators, $a b\left(\partial r_{i} / \partial x_{j}\right)$; hence, the module $A(G)$ is presented by the Alexander matrix. When $G_{\mathrm{ab}}$ is torsion-free, a method for finding a presentation for $B(G)$ is outlined in [41]; an explicit presentation is not known even in the case when $G$ is a link group, but there is an algorithm for producing such a presentation in the case when $G$ is an arrangement group, see [17].

2.4. Functoriality properties. The assignments $G \leadsto G_{\mathrm{ab}}$ and $G \leadsto B(G)$ are functorial and compatible with one another, in a sense that we now make precise. For more background information on some of this material, we refer to [8, Ch. III]. 
First consider a ring map, $\varphi: R \rightarrow S$. We say that a map $\psi: M \rightarrow N$ from an $R$-module $M$ to an $S$-module $N$ covers $\varphi$ (or, for short, that $\psi$ is a $\varphi$-morphism) if $\psi(\mathrm{rm})=\varphi(r) \psi(m)$ for all $r \in R$ and $m \in M$. Such a map $\psi$ can be viewed as the composite

$$
M \longrightarrow N_{\varphi} \longrightarrow N \text {, }
$$

where $N_{\varphi}$ is the $R$-module obtained from $N$ by restriction of scalars via $\varphi$, the first arrow is the set map $\psi$ viewed as an $R$-linear map, and the second arrow is the identity map of $N$, thought of as covering the ring map $\varphi$.

Now let $\alpha: G \rightarrow H$ be a group homomorphism. Then $\alpha$ extends linearly to a ring map, $\tilde{\alpha}: \mathbb{Z}[G] \rightarrow \mathbb{Z}[H]$. The assignment $G \leadsto \mathbb{Z}[G], \alpha \leadsto \tilde{\alpha}$ is functorial, and takes injections to injections and surjections to surjections.

The map $\alpha$ also restricts to homomorphisms $G^{\prime} \rightarrow H^{\prime}$ and $G^{\prime \prime} \rightarrow H^{\prime \prime}$, and thus induces homomorphisms $G / G^{\prime} \rightarrow H / H^{\prime}$ and $G^{\prime} / G^{\prime \prime} \rightarrow H^{\prime} / H^{\prime \prime}$, which we will denote by $\alpha_{\mathrm{ab}}: G_{\mathrm{ab}} \rightarrow H_{\mathrm{ab}}$ and $B(\alpha): B(G) \rightarrow B(H)$, respectively. If $\beta: H \rightarrow K$ is another homomorphism, then clearly $\beta_{\mathrm{ab}} \circ \alpha_{\mathrm{ab}}=(\beta \circ \alpha)_{\mathrm{ab}}$ and $B(\beta) \circ B(\alpha)=B(\beta \circ \alpha)$. If $\alpha$ is surjective, then $B(\alpha)$ is also surjective, but if $\alpha$ is injective, $B(\alpha)$ need not be injective.

Example 2.2. Let $G=\left\langle x_{1}, x_{2} \mid x_{1} x_{2} x_{1}=x_{2} x_{1} x_{2}\right\rangle$, so that $G_{\mathrm{ab}}=\mathbb{Z}$. We then have $G^{\prime}=F_{2}$ and $B\left(G^{\prime}\right)=\mathbb{Z}\left[\mathbb{Z}^{2}\right]$, whereas $B(G)=\mathbb{Z}\left[t^{ \pm 1}\right] /\left(t^{2}-t+1\right)$. Thus, if $\alpha: G^{\prime} \hookrightarrow G$ is the inclusion, the map $B(\alpha)$ is not injective.

Given a homomorphism $\alpha: G \rightarrow H$, let $\tilde{\alpha}_{\mathrm{ab}}: \mathbb{Z}\left[G_{\mathrm{ab}}\right] \rightarrow \mathbb{Z}\left[H_{\mathrm{ab}}\right]$ be the linear extension of $\alpha_{\mathrm{ab}}$ to group rings. The map $B(\alpha): B(G) \rightarrow B(H)$ can then be interpreted as a map of modules covering $\tilde{\alpha}_{\mathrm{ab}}$. Alternatively, let $B(H)_{\alpha}$ be the $\mathbb{Z}\left[G_{\mathrm{ab}}\right]$-module obtained from $B(H)$ by restriction of scalars via $\tilde{\alpha}_{\mathrm{ab}}$. The map $B(\alpha)$ can then be viewed as the composite $B(G) \rightarrow B(H)_{\alpha} \rightarrow B(H)$, where the first arrow is a $\mathbb{Z}\left[G_{\mathrm{ab}}\right]$-linear map and the second arrow is the identity map of $B(H)$, viewed as covering the ring map $\tilde{\alpha}_{\mathrm{ab}}$.

Here is a topological interpretation. Let $f: X \rightarrow Y$ be a continuous maps between connected CW-complexes; without loss of essential generality, we may assume $f$ is cellular and basepoint-preserving. Let $f_{\sharp}: \pi_{1}\left(X, x_{0}\right) \rightarrow \pi_{1}\left(Y, y_{0}\right)$ be the induced homomorphism on fundamental groups, and let $f^{\mathrm{ab}}: X^{\mathrm{ab}} \rightarrow Y^{\mathrm{ab}}$ be the lift to universal abelian covers. It is readily seen that the morphism $B\left(f_{\sharp}\right): B\left(\pi_{1}(X)\right) \rightarrow B\left(\pi_{1}(Y)\right)$ coincides with the induced homomorphism in first homology, $f_{*}: H_{1}\left(X^{\mathrm{ab}} ; \mathbb{Z}\right) \rightarrow H_{1}\left(Y^{\mathrm{ab}} ; \mathbb{Z}\right)$.

Likewise, there is an induced morphism on Alexander modules, $A(\alpha): A(G) \rightarrow A(H)$, which covers $\tilde{\alpha}_{\mathrm{ab}}$ and admits a similar topological interpretation. The restriction of $A(\alpha)$ to $B(G)$ coincides with $B(\alpha)$, and induces the map $\tilde{\alpha}_{\mathrm{ab}}$ on augmentation ideals, thereby showing that the Crowell exact sequence (12) is natural with respect to group homomorphisms.

\section{The rational Derived Series and the rational Alexander invariant}

In this section we discuss the rational versions of the derived series and of the Alexander invariant. 
3.1. The rational derived series. For a subset $S$ of a group $G$, we let $\sqrt{S}=\{g \in G \mid$ $g^{m} \in S$ for some $\left.m \in \mathbb{N}\right\}$ be its isolator. Clearly, $S \subseteq \sqrt{S}$ and $\sqrt{\sqrt{S}}=\sqrt{S}$; moreover, if $\alpha: G \rightarrow H$ is a homomorphism, then

$$
\alpha(\sqrt{S}) \subseteq \sqrt{\alpha(S)} .
$$

The following notion was introduced by Harvey in [32], and further studied by Cochran and Harvey in $[12,13]$. The rational derived series of a group $G$, denoted $\left\{G_{\mathbb{Q}}^{(r)}\right\}_{r \geqslant 0}$, is defined inductively by setting $G_{\mathbb{Q}}^{(0)}=G$ and

$$
G_{\mathrm{Q}}^{(r)}=\sqrt{\left[G_{\mathrm{Q}}^{(r-1)}, G_{\mathrm{Q}}^{(r-1)}\right]} .
$$

Using (5), (14), and induction on $r$, it is readily seen that the terms of this series are fully invariant subgroups. In particular, the rational derived series is a normal series, a property also noted in [32, Lemma 3.2]. Furthermore, the successive quotients, $G_{\mathbb{Q}}^{(r)} / G_{\mathbb{Q}}^{(r+1)}$, are torsion-free abelian groups; in fact, as shown in [32, Lemma 3.5],

$$
G_{\mathrm{Q}}^{(r)} / G_{\mathrm{Q}}^{(r+1)} \cong\left(G_{\mathrm{Q}}^{(r)}\right)_{\mathrm{abf}} \cdot
$$

In particular, $G / G_{\odot}^{\prime}$ is equal to $G_{\mathrm{abf}}=G_{\mathrm{ab}} / \operatorname{Tors}\left(G_{\mathrm{ab}}\right)$, the maximal torsion-free abelian quotient of $G$, showing that $G_{\mathrm{Q}}^{\prime}$ is the kernel of the projection map abf: $G \rightarrow G_{\mathrm{abf}}$. Since $G^{\prime}=\operatorname{ker}\left(\mathrm{ab}: G \rightarrow G_{\mathrm{ab}}\right)$, we obtain a short exact sequence,

$$
1 \longrightarrow G^{\prime} \longrightarrow G_{\mathrm{Q}}^{\prime} \longrightarrow \operatorname{Tors}\left(G_{\mathrm{ab}}\right) \longrightarrow 1 \text {. }
$$

In particular, if $G_{\mathrm{ab}}$ is torsion-free, then $G^{\prime}=G_{\mathrm{Q}}^{\prime}$.

Remark 3.1. In $[22,20]$, the group $G_{Q}^{\prime}=\operatorname{ker}\left(\operatorname{abf}: G \rightarrow G_{\text {abf }}\right)$ is called the Johnson kernel of $G$, and is denoted by $K_{G}$. The motivation for this terminology is that, in the case when $G=\mathscr{T}_{g}$ is the Torelli group of a surface of genus $g \geqslant 3$, the subgroup $K_{G} \triangleleft \mathscr{T}_{g}$ is the subgroup generated by Dehn twists along separating simple closed curves-a subgroup introduced and studied by D. Johnson in the 1980s; see [11] for more on this.

It is also known that the quotient groups $G / G_{\odot}^{(r+1)}$ are poly-torsion-free-abelian (PTFA) groups, see [32, Corollary 3.6]. Cleary, $G^{(r)} \subseteq G_{0}^{(r)}$ for all $r$, but the inclusions are strict in general. Nevertheless, if all the quotients $G^{(r)} / G^{(r+1)}$ are torsion-free-which is the case when $G$ is a finitely generated free group, or a knot group-then $G^{(r)}=G_{\mathbb{Q}}^{(r)}$ for all $r$, cf. [32, Corollary 3.7].

3.2. The rational Alexander invariant. By analogy with the classical definition, we define the rational Alexander invariant of a group $G$ to be the quotient

$$
B_{\mathbb{Q}}(G):=G_{\mathbb{Q}}^{\prime} / G_{Q}^{\prime \prime}
$$


viewed as a module over $\mathbb{Z}\left[G_{\mathrm{abf}}\right]$, where recall $G_{\mathrm{Q}}^{\prime}=\sqrt{G^{\prime}}$ and $G_{\mathrm{Q}}^{\prime \prime}=\sqrt{\left[\sqrt{G^{\prime}}, \sqrt{G^{\prime}}\right]} \triangleleft G_{\mathrm{Q}}^{\prime}$. The module structure on $B_{\mathbb{Q}}(G)$ is induced by conjugation in the maximal torsion-free metabelian quotient, $G / G_{\mathrm{Q}}^{\prime \prime}$, via the exact sequence

$$
0 \longrightarrow G_{\mathrm{Q}}^{\prime} / G_{\mathrm{Q}}^{\prime \prime} \longrightarrow G / G_{\mathrm{Q}}^{\prime \prime} \longrightarrow G / G_{\mathrm{Q}}^{\prime} \longrightarrow 0 \text {. }
$$

That is, $g G_{\odot}^{\prime} \cdot x G_{\odot}^{\prime \prime}=g x g^{-1} G_{\odot}^{\prime \prime}$ for all $g \in G$ and $x \in G_{\odot}^{\prime}$, with the action of $G / G_{\odot}^{\prime}=G_{\text {abf }}$ extended $\mathbb{Z}$-linearly to the whole of $\mathbb{Z}\left[G_{\text {abf }}\right]$.

Given a group homomorphism $\alpha: G \rightarrow H$, the maps $\alpha^{\prime}: G_{\mathrm{Q}}^{\prime} \rightarrow H_{\mathrm{Q}}^{\prime}$ and $\alpha^{\prime \prime}: G_{\mathrm{Q}}^{\prime \prime} \rightarrow$ $H_{\mathrm{Q}}^{\prime \prime}$ induce a morphism $B_{\mathrm{Q}}(\alpha): B_{\mathrm{Q}}(G) \rightarrow B_{\mathrm{Q}}(H)$ between rational Alexander invariants. It is readily seen that the assignments $G \leadsto G_{\text {abf }}$ and $G \leadsto B_{\mathrm{Q}}(G)$ are functorial and compatible with one another, in a sense similar to the one described in $\$ 2.2$.

Pursuing our analogy with the classical situation, we also define the rational Alexander module of $G$ to be the $\mathbb{Z}\left[G_{\text {abf }}\right]$-module $A_{\mathbb{Q}}(G):=\mathbb{Z}\left[G_{\text {abf }}\right] \otimes_{\mathbb{Z}[G]} I(G)$.

3.3. Topological interpretation. Let $X$ be a connected CW-complex with $\pi_{1}(X)=G$, and let $q_{0}: X^{\text {abf }} \rightarrow X$ be the maximal torsion-free abelian cover of $X$. We then have a commuting diagram of regular covers,

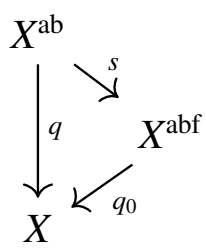

where $s: X^{\mathrm{ab}} \rightarrow X^{\mathrm{abf}}$ is an abelian cover with deck group Tors $\left(G_{\mathrm{ab}}\right)$. Clearly, if $G_{\mathrm{ab}}$ is finitely generated, then $s$ is a finite cover. Note that $H_{\bullet}\left(X^{\mathrm{abf}} ; \mathbb{Z}\right)$ is a module over $\mathbb{Z}\left[G_{\mathrm{abf}}\right]$, with module structure induced by the action of $G_{\mathrm{abf}}$ on $X^{\text {abf }}$ by deck transformations.

Lemma 3.2. With notation as above,

(1) $B_{\mathrm{Q}}(G) \cong H_{1}\left(X^{\mathrm{abf}} ; \mathbb{Z}\right) / \mathbb{Z}$-Tors, as $\mathbb{Z}\left[G_{\mathrm{abf}}\right]$-modules.

(2) $B_{\mathbb{Q}}(G) \otimes \mathbb{Q} \cong H_{1}\left(X^{\mathrm{abf}} ; \mathbb{Q}\right)$, as $\mathbb{Q}\left[G_{\mathrm{abf}}\right]$-modules.

(3) If $G_{\mathrm{ab}}$ is torsion-free, then $B_{\mathrm{Q}}(G) \cong B(G) / \mathbb{Z}$-Tors, as $\mathbb{Z}\left[G_{\mathrm{ab}}\right]$-modules.

Proof. From definition (18) and formula (16), we have that $B_{\mathrm{Q}}(G)=\left(G_{\mathrm{Q}}^{\prime}\right)_{\mathrm{abf}}$. Since $G_{\mathbb{Q}}^{\prime}=\pi_{1}\left(X^{\text {abf }}\right)$, the first claim follows. Tensoring both sides with $\mathbb{Q}$ yields the second claim. The third claim follows at once from the first one.

Remark 3.3. In view of Lemma 3.2, part (1) and the discussion from Remark 3.1, the rational Alexander invariant $B_{Q}(G)$ may be viewed as the torsion-free abelianization of the Johnson kernel $K_{G}$. In a related vein, we considered in [53] the $\mathbb{Q}\left[G_{\text {abf }}\right]$-module $\tilde{B}(G):=H_{1}\left(G_{\mathbb{Q}} ; \mathbb{Q}\right)$, which was called there the reduced Alexander invariant of $G$. In view of Lemma 3.2, part (2), this module is isomorphic to $B_{\mathbb{Q}}(G) \otimes \mathbb{Q}$. 
Let $\left(C \cdot\left(X^{\mathrm{ab}} ; \mathbb{Z}\right), \partial^{\mathrm{ab}}\right)$ be the $\mathbb{Z}\left[G_{\mathrm{ab}}\right]$-equivariant chain complex of $X^{\mathrm{ab}}$ from (9). We denote by $v: G_{\mathrm{ab}} \rightarrow G_{\mathrm{abf}}$ the projection map, and we let $\tilde{v}: \mathbb{Z}\left[G_{\mathrm{ab}}\right] \rightarrow \mathbb{Z}\left[G_{\mathrm{abf}}\right]$ be its linear extension to group rings. The $\mathbb{Z}\left[G_{\mathrm{abf}}\right]$-equivariant chain complex of $X^{\text {abf }}$, denoted $\left(C .\left(X^{\mathrm{abf}} ; \mathbb{Z}\right), \partial^{\mathrm{abf}}\right)$, may be obtained from (9) by extension of scalars, via the ring map $\tilde{v}$; in particular, $\partial^{\mathrm{abf}}=\partial^{\mathrm{ab}} \otimes_{\mathbb{Z}\left[G_{\mathrm{ab}}\right]} \mathbb{Z}\left[G_{\mathrm{abf}}\right]$.

Let also $F_{0}=q_{0}^{-1}\left(x_{0}\right)$ be the fiber of the cover $q_{0}: X^{\text {abf }} \rightarrow X$ over a basepoint $x_{0} \in X$, and let $I_{0}(G)$ be the kernel of the augmentation map $\varepsilon: \mathbb{Z}\left[G_{\text {abf }}\right] \rightarrow \mathbb{Z}$. Proceeding as in $\S 2.3$, we obtain the following lemma, which summarizes the properties of the rational Alexander module, and its relationship to the rational Alexander invariant.

Lemma 3.4. With notation as above,

(1) $A_{\mathbb{Q}}(G) \otimes \mathbb{Q}=H_{1}\left(X^{\mathrm{abf}}, F_{0} ; \mathbb{Q}\right)$.

(2) $A_{\mathbb{Q}}(G) \otimes \mathbb{Q}$ is the cokernel of the map $\partial_{2}^{\mathrm{abf}} \otimes \mathbb{Q}: C_{2}\left(X^{\mathrm{abf}} ; \mathbb{Q}\right) \rightarrow C_{1}\left(X^{\mathrm{abf}} ; \mathbb{Q}\right)$.

(3) The homology exact sequence of the pair $\left(X^{\mathrm{abf}}, F_{0}\right)$ yields a short exact sequence of $\mathbb{Q}\left[G_{\mathrm{abf}}\right]$-modules,

$$
0 \longrightarrow B_{\mathbb{Q}}(G) \otimes \mathbb{Q} \longrightarrow A_{\mathbb{Q}}(G) \otimes \mathbb{Q} \longrightarrow I_{0}(G) \otimes \mathbb{Q} \longrightarrow 0 .
$$

Sequence (21) may be viewed as the rational analogue of Crowell's exact sequence (12), and enjoys a similar naturality property with respect to group homomorphisms.

3.4. Relating the Alexander invariants. We conclude this section with a comparison between the two kinds of Alexander invariants defined so far: $B(G)$, viewed as a $\mathbb{Z}\left[G_{\mathrm{ab}}\right]$ module, and $B_{\mathbb{Q}}(G)$, viewed as a $\mathbb{Z}\left[G_{\mathrm{abf}}\right]$-module. The comparison is done via the natural projection $v: G_{\mathrm{ab}} \rightarrow G_{\mathrm{abf}}$ and its extension to a ring map, $\tilde{v}: \mathbb{Z}\left[G_{\mathrm{ab}}\right] \rightarrow \mathbb{Z}\left[G_{\mathrm{abf}}\right]$.

Proposition 3.5. For a group $G$, the following hold.

(1) The inclusion $G^{\prime} \hookrightarrow G_{\mathrm{Q}}^{\prime}$ induces a functorial $\tilde{v}$-morphism, $\kappa: B(G) \rightarrow B_{\mathrm{Q}}(G)$.

(2) Suppose Tors $\left(G_{\mathrm{ab}}\right)$ is finite. Then the map $\kappa \otimes \mathbb{Q}: B(G) \otimes \mathbb{Q} \rightarrow B_{\mathbb{Q}}(G) \otimes \mathbb{Q}$ is surjective.

(3) Suppose $G_{\mathrm{ab}}$ is torsion-free. Then the map $\kappa \otimes \mathbb{Q}: B(G) \otimes \mathbb{Q} \rightarrow B_{\mathbb{Q}}(G) \otimes \mathbb{Q}$ is an isomorphism.

Proof. (1) The inclusion $G^{\prime} \hookrightarrow G_{\mathrm{Q}}^{\prime}$ restricts to a map $G^{\prime \prime} \hookrightarrow G_{\mathrm{Q}}^{\prime \prime}$, and thus induces a group homomorphism, $G^{\prime} / G^{\prime \prime} \rightarrow G_{\mathrm{Q}}^{\prime} / G_{\mathrm{Q}}^{\prime \prime}, g G^{\prime \prime} \mapsto g G_{\mathrm{Q}}^{\prime \prime}$, which is functorial in $G$. This homomorphism can be viewed as a map $\kappa:=\kappa^{G}: B(G) \rightarrow B_{\mathrm{Q}}(G)$ which covers the ring map $\tilde{v}$ and satisfies $\kappa^{H} \circ B(\alpha)=B_{\odot}(\alpha) \circ \kappa^{G}$, for all homomorphisms $\alpha: G \rightarrow H$. We may also view $\kappa$ as the composite $B(G) \rightarrow B_{\mathbb{Q}}(G)_{\tilde{v}} \rightarrow B_{Q}(G)$, where the first arrow is a $\mathbb{Z}\left[G_{\mathrm{ab}}\right]$-linear map and the second arrow is the identity map of $B_{\mathbb{Q}}(G)$, viewed as covering the map $\tilde{v}$.

Alternatively, recall from (20) that the cover $q: X^{\mathrm{ab}} \rightarrow X$ factors through a cover $s: X^{\mathrm{ab}} \rightarrow X^{\mathrm{abf}}$, so that $q_{0} \circ s=q$. The composite

$$
H_{1}\left(X^{\mathrm{ab}} ; \mathbb{Z}\right) \stackrel{s_{*}}{\longrightarrow} H_{1}\left(X^{\mathrm{abf}} ; \mathbb{Z}\right) \longrightarrow H_{1}\left(X^{\mathrm{abf}} ; \mathbb{Z}\right) / \mathbb{Z} \text {-Tors }
$$


then coincides with the morphism $\kappa$ defined above.

(2) From the last description, it follows that the homomorphism $s_{*} \otimes \mathbb{Q}: H_{1}\left(X^{\mathrm{ab}} ; \mathbb{Q}\right) \rightarrow$ $H_{1}\left(X^{\text {abf }} ; \mathbb{Q}\right)$ coincides with $\kappa \otimes \mathbb{Q}$. Now, if Tors $\left(G_{\mathrm{ab}}\right)$ is finite, then $s: X^{\mathrm{ab}} \rightarrow X^{\text {abf }}$ is a finite cover, and so the transfer map, $\tau: H_{1}\left(X^{\mathrm{abf}} ; \mathbb{Q}\right) \rightarrow H_{1}\left(X^{\mathrm{ab}} ; \mathbb{Q}\right)$, provides a splitting for $s_{*}$. Therefore, $\kappa \otimes \mathbb{Q}$ is surjective.

(3) If $G_{\mathrm{ab}}$ is torsion-free, then $\operatorname{ker} s_{*}=0$, and so $\kappa \otimes \mathbb{Q}=s_{*} \otimes \mathbb{Q}$ is an isomorphism.

If $G$ is a finitely generated free group, or a knot group, then, by the discussion in $\S 3.1$, the map $\kappa: B(G) \rightarrow B_{Q}(G)$ is a $\tilde{v}$-isomorphism. On the other hand, as Example 3.6 below shows, the condition that $G_{\mathrm{ab}}$ be torsion-free is necessary for part (3) of Proposition 3.5 to hold. Moreover, as Example 3.7 shows, the map $\kappa: B(G) \rightarrow B_{\mathbb{Q}}(G)$ itself need not be an isomorphism, even when $G_{\mathrm{ab}}$ is torsion-free.

Example 3.6. Let $G=\mathbb{Z}_{2} * \mathbb{Z}_{2}=\left\langle x_{1}, x_{2} \mid x_{1}^{2}, x_{2}^{2}\right\rangle$. Then $G^{\prime}=\mathbb{Z}=\left\langle\left[x_{1}, x_{2}\right]\right\rangle$ and $G^{\prime \prime}=\{1\}$, whence $B(G)=\mathbb{Z}$, whereas $G_{Q}^{\prime}=G_{Q}^{\prime \prime}=G$, whence $B_{Q}(G)=0$.

Example 3.7. Let $G=\left\langle x_{1}, x_{2} \mid\left[x_{1}, x_{2}\right]^{n}\right\rangle$. Then $B(G)=\mathbb{Z}\left[x_{1}^{ \pm 1}, x_{2}^{ \pm 1}\right] /(n) \cong \mathbb{Z}_{n}\left[x_{1}^{ \pm 1}, x_{2}^{ \pm 1}\right]$ is non-zero if $n \geqslant 2$, whereas $B_{\mathrm{Q}}(G)=0$.

\section{The Derived $p$-Series AND THE MOd- $p$ Alexander invariant}

We now review the mod- $p$ version of the derived series and introduce the corresponding mod- $p$ Alexander invariant.

4.1. The derived $p$-series. Fix a prime $p$. For a subset $S$ of a group $G$, we let its $p$-th power be the set $S^{p}:=\left\{g \in G \mid g=x^{p}\right.$ for some $\left.x \in S\right\}$. Clearly, if $\varphi: G \rightarrow H$ is a homomorphism, then

$$
\varphi\left(S^{p}\right) \subseteq(\varphi(S))^{p}
$$

Following Stallings [60], Cochran and Harvey [14, 15], and Lackenby [36], we define the derived $p$-series of $G$, denoted $\left\{G_{p}^{(r)}\right\}_{r \geqslant 0}$, by

$$
G_{p}^{(0)}=G, \quad G_{p}^{(r)}=\left\langle\left(G_{p}^{(r-1)}\right)^{p},\left[G_{p}^{(r-1)}, G_{p}^{(r-1)}\right]\right\rangle .
$$

Using formulas (5) and (23) and induction on $r$, it is readily seen that the terms of this series are fully invariant subgroups. Moreover, each subgroup $G_{p}^{(r)}$ is a normal subgroup of $G$ of index a power of $p$, see [60], and $G_{p}^{(r-1)} / G_{p}^{(r)} \cong H_{1}\left(G_{p}^{(r-1)} ; \mathbb{Z}_{p}\right)$, see [36]. In particular, $G / G_{p}^{\prime}=H_{1}\left(G ; \mathbb{Z}_{p}\right)$ is the maximal elementary $p$-abelian quotient of $G$.

Example 4.1. Suppose $G$ is abelian, Then clearly $G_{p}^{(r)}=G^{p^{r}}$. In particular, if $G$ is elementary $p$-abelian, then $G_{p}^{(r)}=\{1\}$ for all $r \geqslant 1$.

The derived $p$-series can be characterized as the fastest descending normal (and even subnormal) series for which the successive quotients are $\mathbb{Z}_{p}$-vector spaces, cf. [60]. The next result captures some of the salient features of this series. 
Lemma 4.2 ([14]). For a group $G$, a prime $p$, and an integer $r \geqslant 1$, the following hold.

(1) $G_{p}^{(r)}=\operatorname{ker}\left(G_{p}^{(r-1)} \rightarrow\left(G_{p}^{(r-1)}\right)_{\mathrm{ab}} \otimes \mathbb{Z}_{p}\right)$.

(2) $G_{p}^{(r-1)} / G_{p}^{(r)} \cong H_{1}\left(G ; \mathbb{Z}_{p}\left[G / G_{p}^{(r-1)}\right]\right)$, as right $\mathbb{Z}_{p}\left[G / G_{p}^{(r-1)}\right]$-modules.

(3) If $G$ is finitely generated, then $G / G_{p}^{(r)}$ is a finite p-group, with all elements having order dividing $p^{r}$.

4.2. The mod- $p$ Alexander invariant. The first two $p$-derived subgroups of $G$ are given by $G_{p}^{\prime}=\left\langle G^{p}, G^{\prime}\right\rangle$ and $G_{p}^{\prime \prime}=\left\langle G^{p^{2}},\left(G^{\prime}\right)^{p},\left[G^{p}, G^{p}\right],\left[G^{p}, G^{\prime}\right], G^{\prime \prime}\right\rangle$; moreover, $G_{p}^{\prime \prime} \triangleleft G_{p}^{\prime}$. We define the mod-p Alexander invariant of $G$ to be the quotient of these two subgroups,

$$
B_{p}(G):=G_{p}^{\prime} / G_{p}^{\prime \prime}
$$

and view it as a module over the group-ring $\Lambda_{p}:=\mathbb{Z}_{p}\left[H_{1}\left(G ; \mathbb{Z}_{p}\right)\right]$. The module structure is induced by conjugation in the maximal metabelian $p$-quotient, $G / G_{p}^{\prime \prime}$, via the exact sequence

$$
0 \longrightarrow G_{p}^{\prime} / G_{p}^{\prime \prime} \longrightarrow G / G_{p}^{\prime \prime} \longrightarrow G / G_{p}^{\prime} \longrightarrow 0
$$

That is, $g G_{p}^{\prime} \cdot x G_{p}^{\prime \prime}=g x g^{-1} G_{p}^{\prime \prime}$ for all $g \in G$ and $x \in G_{p}^{\prime}$, with the action of $G / G_{p}^{\prime}=$ $H_{1}\left(G ; \mathbb{Z}_{p}\right)$ on the $\mathbb{Z}_{p}$-vector space $G_{p}^{\prime} / G_{p}^{\prime \prime}$ extended $\mathbb{Z}_{p}$-linearly to the whole of $\Lambda_{p}$. By Lemma 4.2, parts (1) and (2), we have that

$$
B_{p}(G)=\left(G_{p}^{\prime}\right)_{\mathrm{ab}} \otimes \mathbb{Z}_{p}=H_{1}\left(G_{p}^{\prime} ; \mathbb{Z}_{p}\right) \cong H_{1}\left(G ; \Lambda_{p}\right)
$$

as a $\Lambda_{p}$-module. Let $b_{i}^{p}(G):=\operatorname{dim}_{\mathbb{Z}_{p}} H_{i}\left(G ; \mathbb{Z}_{p}\right)$ be the $i$-th mod- $p$ Betti numbers of $G$. If $G$ is finitely generated, then the $\mathbb{Z}_{p}$-vector space $B_{p}(G)$ is finite-dimensional, and we have $\operatorname{dim}_{\mathbb{Z}_{p}} B_{p}(G)=b_{1}^{p}\left(G_{p}^{\prime}\right)$.

We also define the mod-p Alexander module of $G$ as $A_{p}(G)=\Lambda_{p} \otimes_{\mathbb{Z}[G]} I(G)$, with $\Lambda_{p}$-module structure given by multiplication on the left factor. Given a group homomorphism $\alpha: G \rightarrow H$, we let $B_{p}(\alpha): B_{p}(G) \rightarrow B_{p}(H)$ be the morphism between mod- $p$ Alexander invariants induced by the restriction $\alpha^{\prime}: G_{p}^{\prime} \rightarrow H_{p}^{\prime}$. It is readily seen that the assignments $G \leadsto H_{1}\left(G ; \mathbb{Z}_{p}\right)$ and $G \leadsto B_{p}(G)$ are functorial and compatible with one another, in a manner similar to the one described in $\$ 2.2$.

4.3. Topological interpretation. The mod- $p$ Alexander invariant admits the following interpretation in terms of covering spaces. Let $X$ be a connected $\mathrm{CW}$-complex with $\pi_{1}(X)=G$, and let $q_{p}: X^{(p)} \rightarrow X$ be the $p$-congruence cover of $X$; that is, the regular $H_{1}\left(X ; \mathbb{Z}_{p}\right)$-cover classified by the composite

$$
\pi_{1}(X) \stackrel{\mathrm{ab}}{\longrightarrow} H_{1}(X ; \mathbb{Z}) \stackrel{v_{p}}{\longrightarrow} H_{1}\left(X ; \mathbb{Z}_{p}\right),
$$


where $v_{p}$ is the coefficient homomorphism induced by the projection $\mathbb{Z} \rightarrow \mathbb{Z}_{p}$. We then have a commuting diagram of regular covers,

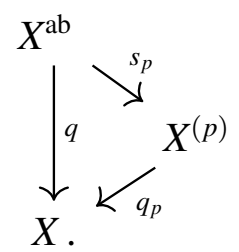

By construction, $\pi_{1}\left(X^{(p)}\right)=G_{p}^{\prime}$; therefore, $B_{p}(G) \cong H_{1}\left(X^{(p)} ; \mathbb{Z}_{p}\right)$, with $\Lambda_{p}$-module structure induced by the action of $H_{1}\left(X ; \mathbb{Z}_{p}\right)$ by deck transformations. Proceeding as in $\$ 2.3$, we may identify the mod- $p$ Alexander invariant of $G$ with the cokernel of the boundary map $\partial_{2}^{p}: C_{2}\left(X^{(p)} ; \mathbb{Z}_{p}\right) \rightarrow C_{1}\left(X^{(p)} ; \mathbb{Z}_{p}\right)$. Moreover, $A_{p}(G)=H_{1}\left(X^{(p)}, F_{p} ; \mathbb{Z}_{p}\right)$, where $F_{p}=q_{p}^{-1}\left(x_{0}\right)$. The $\mathbb{Z}_{p}$-homology exact sequence of the pair $\left(X^{(p)}, F_{p}\right)$ now yields a natural short exact sequence of $\Lambda_{p}$-modules,

$$
0 \longrightarrow B_{p}(G) \longrightarrow A_{p}(G) \longrightarrow I_{p}(G) \longrightarrow 0,
$$

where $I_{p}(G)=\operatorname{ker}\left(\varepsilon: \Lambda_{p} \rightarrow \mathbb{Z}_{p}\right)$ is the augmentation ideal. Sequence (30) is natural, and may be viewed as the mod- $p$ analogue of Crowell's exact sequence (12).

4.4. A comparison map. The next lemma provides a functorial comparison map between the reduction mod- $p$ of the usual Alexander invariant and its mod- $p$ version. Let $\tilde{v}_{p}: \mathbb{Z}_{p}\left[H_{1}(G ; \mathbb{Z})\right] \rightarrow \mathbb{Z}_{p}\left[H_{1}\left(G ; \mathbb{Z}_{p}\right)\right]$ be the linear extension of the coefficient homomorphism $v_{p}: H_{1}(G ; \mathbb{Z}) \rightarrow H_{1}\left(G ; \mathbb{Z}_{p}\right)$ to group rings.

Lemma 4.3. The inclusion $G^{\prime} \hookrightarrow G_{p}^{\prime}$ induces a functorial $\tilde{v}_{p}$-morphism, $\kappa_{p}: B(G) \otimes$ $\mathbb{Z}_{p} \rightarrow B_{p}(G)$.

Proof. The inclusion $G^{\prime} \hookrightarrow G_{p}^{\prime}$ restricts to a map $G^{\prime \prime} \hookrightarrow G_{p}^{\prime \prime}$, and thus induces a group homomorphism, $G^{\prime} / G^{\prime \prime} \rightarrow G_{p}^{\prime} / G_{p}^{\prime \prime}$. This map factors through a homomorphism, $G^{\prime} / G^{\prime \prime} \otimes$ $\mathbb{Z}_{p} \rightarrow G_{p}^{\prime} / G_{p}^{\prime \prime}$, which can can be viewed as a $\tilde{v}_{p}$-morphism, $\kappa_{p}:=\kappa_{p}^{G}: B(G) \otimes \mathbb{Z}_{p} \rightarrow$ $B_{p}(G)$. Clearly, $\kappa_{p}^{H} \circ B(\alpha)=B_{p}(\alpha) \circ \kappa_{p}^{G}$, for all homomorphisms $\alpha: G \rightarrow H$.

We may interpret the map $\kappa_{p}$ as the homomorphism $\left(s_{p}\right)_{*}: H_{1}\left(X^{\mathrm{ab}} ; \mathbb{Z}_{p}\right) \rightarrow H_{1}\left(X^{(p)} ; \mathbb{Z}_{p}\right)$ induced in first homology by the cover $s_{p}$ from diagram (29). As illustrated in the examples below, the map $\kappa_{p}$ is neither injective nor surjective, in general.

Example 4.4. Let $X=\bigvee^{n} S^{1}$ be a wedge of $n \geqslant 2$ circles. Then $X^{(p)} \simeq \bigvee^{m} S^{1}$, where $m=p^{n}(n-1)+1$. Identifying $F_{n}=\pi_{1}(X)$, we find that $B_{p}\left(F_{n}\right)=H_{1}\left(X^{(p)}, \mathbb{Z}_{p}\right)=\mathbb{Z}_{p}^{m}$. On the other hand, we infer from Example 4.5 that $B\left(F_{2}\right) \otimes \mathbb{Z}_{p}=\mathbb{Z}_{p}\left[\mathbb{Z}^{2}\right]$; hence, the map $\kappa_{p}: B\left(F_{2}\right) \otimes \mathbb{Z}_{p} \rightarrow B_{p}\left(F_{2}\right)$ is not injective.

Example 4.5. Suppose $G$ is abelian. Then $B(G)=B_{\mathbb{Q}}(G)=0$, yet $B_{p}(G)=G^{p} / G^{p^{2}}$, which is non-trivial in general; for instance, $B_{p}\left(\mathbb{Z}^{n}\right)=\mathbb{Z}_{p}^{n}$. In fact, the mod- $p$ Alexander 
invariant may be able to distinguish groups for which the other two kinds of Alexander invariants coincide; for example, $B_{p}\left(\mathbb{Z}_{p} \oplus \mathbb{Z}_{p}\right)=0$, yet $B_{p}\left(\mathbb{Z}_{p^{2}}\right)=\mathbb{Z}_{p}$.

\section{Associated GRADEd Lie algEbras}

In this section we consider three types of graded Lie algebras associated a group $G$ the usual one, its rational version, and its mod- $p$ version-and review some of their salient features.

5.1. Lower central series and associated graded Lie algebra. The lower central series (LCS) of a group $G$, denoted $\left\{\gamma_{n}(G)\right\}_{n \geqslant 1}$, is defined inductively by

$$
\gamma_{1}(G)=G \text { and } \gamma_{n+1}(G)=\left[G, \gamma_{n}(G)\right] \text {. }
$$

This an N-series, in the sense of Lazard [37]; that is to say, $\left[\gamma_{m}(G), \gamma_{n}(G)\right] \subseteq \gamma_{m+n}(G)$, for all $m, n \geqslant 1$, see for instance $[39,55]$. In particular, the LCS is a central series, i.e., $\left[G, \gamma_{n}(G)\right] \subseteq \gamma_{n+1}(G)$ for all $n$; in fact, it is the fastest descending central series of $G$. Moreover, its terms are fully invariant subgroups of $G$. By definition, the LCS terminates in finitely many steps if the group is nilpotent, and the intersection of the terms of the series is trivial if $G$ is residually nilpotent.

Note that $G^{(n-1)} \subseteq \gamma_{n}(G)$, with equality for $n=1$ and 2. Consequently, every nilpotent group is solvable. As another consequence of this observation, the Alexander invariant $B(G)=G^{\prime} / G^{\prime \prime}$ surjects onto the quotient $\gamma_{2}(G) / \gamma_{3}(G)$.

The successive quotients of the lower central series, $\operatorname{gr}_{n}(G):=\gamma_{n}(G) / \gamma_{n+1}(G)$, are abelian groups. Their direct sum,

$$
\operatorname{gr}(G)=\bigoplus_{n \geqslant 1} \gamma_{n}(G) / \gamma_{n+1}(G),
$$

is the associated graded Lie algebra of $G$. The addition in $\operatorname{gr}(G)$ is induced from the group multiplication, while the Lie bracket is induced from the group commutator; furthermore, this bracket is compatible with the grading. By construction, the Lie algebra $\operatorname{gr}(G)$ is generated by its degree 1 piece, $\operatorname{gr}_{1}(G)=G_{\mathrm{ab}}$; thus, if $G_{\mathrm{ab}}$ is finitely generated, then so are the LCS quotients of $G$. Likewise, the $\mathbb{Q}$-Lie algebra $\operatorname{gr}(G) \otimes \mathbb{Q}$ is generated in degree 1 by the $\mathbb{Q}$-vector space $G_{\mathrm{ab}} \otimes \mathbb{Q}=H_{1}(G ; \mathbb{Q})$. Assume now that the first Betti number, $b_{1}(G)=\operatorname{dim}_{\mathbb{Q}} H_{1}(G ; \mathbb{Q})$, is finite; we may then define the $L C S$ ranks of $G$ as

$$
\phi_{n}(G):=\operatorname{dim}_{\mathbb{Q}} \operatorname{gr}_{n}(G) \otimes \mathbb{Q} .
$$

If $\alpha: G \rightarrow H$ is a group homomorphism, then $\alpha\left(\gamma_{n}(G)\right) \subseteq \gamma_{n}(H)$, and thus $\alpha$ induces a map $\operatorname{gr}(\alpha): \operatorname{gr}(G) \rightarrow \operatorname{gr}(H)$. It is readily seen that this map preserves Lie brackets and that the assignment $\alpha \leadsto \operatorname{gr}(\alpha)$ is functorial. For each $n \geqslant 1$, the quotient group, $\Gamma_{n}:=G / \gamma_{n}(G)$, is a nilpotent group, to wit, the maximal $(n-1)$-step nilpotent quotient of $G$. Since this group is nilpotent, its torsion elements, $\operatorname{Tors}\left(\Gamma_{n}\right)$, form a (characteristic) subgroup; the quotient group, $\Gamma_{n} / \operatorname{Tors}\left(\Gamma_{n}\right)$, is the maximal $(n-1)$-step, torsion-free nilpotent quotient of $G$. 
The associated graded Lie algebra of a group $G$ may be approximated by the associated graded Lie algebras of its solvable quotients, $\operatorname{gr}\left(G / G^{(r)}\right)$. For each $r \geqslant 2$, the quotient map, $G \rightarrow G / G^{(r)}$, induces a surjective morphism, $\operatorname{gr}_{n}(G) \rightarrow \operatorname{gr}_{n}\left(G / G^{(r)}\right)$, which is an isomorphism for $n \leqslant 2^{r}-1$, see [69]. In the case when $r=2$, originally studied by K.-T. Chen in [9], the corresponding Lie algebra, $\operatorname{gr}\left(G / G^{\prime \prime}\right)$, is called the Chen Lie algebra of $G$. Assuming $b_{1}(G)<\infty$, we may define the Chen ranks of $G$ as

$$
\theta_{n}(G):=\phi_{n}\left(G / G^{\prime \prime}\right)=\operatorname{dim}_{\mathbb{Q}} \operatorname{gr}_{n}\left(G / G^{\prime \prime}\right) \otimes \mathbb{Q} .
$$

In view of the above discussion, we have that $\theta_{n}(G) \leqslant \phi_{n}(G)$, with equality for $n \leqslant 3$. We refer to $[45,69,70]$ for detailed treatments of this subject.

5.2. The rational lower central series. The rational version of the lower central series of a group $G$, denoted $\gamma^{\mathbb{Q}}(G)=\left\{\gamma_{n}^{\mathbb{Q}}(G)\right\}_{n \geqslant 1}$, was introduced by Stallings in [59], and further studied in $[4,13,42,55,66]$. The series in defined inductively by

$$
\gamma_{1}^{\mathrm{Q}}(G)=G \text { and } \gamma_{n+1}^{\mathrm{Q}}(G)=\sqrt{\left[G, \gamma_{n}^{\mathrm{Q}}(G)\right]} .
$$

The terms of this series are fully invariant subgroups of $G$. Moreover, as shown in [66], we have that $\gamma_{n}^{\odot}(G)=\sqrt{\gamma_{n}(G)}$, for all $n \geqslant 1$. This implies that $\gamma^{\complement}(G)$ is an N-series (see $[42,55])$, and thus, a central series. In fact, the rational LCS is the most rapidly descending central series whose successive quotients are torsion-free abelian groups. This series terminates in finitely many steps if the group is a torsion-free nilpotent. Moreover, the intersection of the terms of the rational LCS is trivial if and only $G$ is residually torsionfree nilpotent (see [4]). Also note that we have inclusions $G_{\mathrm{Q}}^{(n-1)} \subseteq \gamma_{n}^{\mathrm{Q}}(G)$, with equality for $n=1$ and 2. Consequently, the rational Alexander invariant, $B_{\mathrm{Q}}(G)=G_{\mathrm{Q}}^{\prime} / G_{\mathrm{Q}}^{\prime \prime}$, maps surjectively onto the group $\gamma_{2}^{\mathbb{Q}}(G) / \gamma_{3}^{\mathbb{Q}}(G)$.

The direct sum of the successive quotients of $\gamma^{Q}(G)$,

$$
\operatorname{gr}^{\mathrm{Q}}(G)=\bigoplus_{n \geqslant 1} \gamma_{n}^{\mathrm{Q}}(G) / \gamma_{n+1}^{\mathrm{Q}}(G)
$$

with Lie bracket induced from the group commutator, constitutes the rational associated graded Lie algebra of $G$. If $\alpha: G \rightarrow H$ is a group homomorphism, then $\alpha$ induces a functorial morphism of graded Lie algebras, $\operatorname{gr}^{\mathrm{Q}}(\alpha): \operatorname{gr}^{\mathrm{Q}}(G) \rightarrow \operatorname{gr}^{\mathrm{Q}}(H)$.

Clearly $\gamma_{n}(G) \leqslant \gamma_{n}^{\mathrm{Q}}(G)$ for all $n$. We thus we have an induced map between associated graded Lie algebras, $\Phi=\Phi^{G}: \operatorname{gr}(G) \rightarrow \operatorname{gr}^{\complement}(G)$, which is functorial with respect to group homomorphisms. In degree 1, this map is simply the projection $G_{\mathrm{ab}} \rightarrow G_{\mathrm{abf}}$, with kernel Tors $\left(G_{\mathrm{ab}}\right)$. In [4, Proposition 7.2], Bass and Lubotzky prove the following result.

Proposition 5.1 ([4]). For a group $G$, the following hold.

(1) The map $\Phi: \operatorname{gr}(G) \rightarrow \operatorname{gr}^{\mathrm{Q}}(G)$ has torsion kernel and cokernel in each degree.

(2) The map $\Phi \otimes \mathbb{Q}: \operatorname{gr}(G) \otimes \mathbb{Q} \rightarrow \operatorname{gr}^{\mathbb{Q}}(G) \otimes \mathbb{Q}$ is an isomorphism of graded Lie algebras. 
Recall from $\$ 5.1$ that the Lie algebra $\operatorname{gr}(G)$ is generated by its degree 1 piece, $\operatorname{gr}_{1}(G)=$ $G_{\mathrm{ab}}$. It follows from Proposition 5.1, part (2) that the $\mathbb{Q}$-Lie algebra $\operatorname{gr}^{\mathrm{Q}}(G) \otimes \mathbb{Q}$ is also generated in degree 1 , this time by the $\mathbb{Q}$-vector space $G_{\text {ab }} \otimes \mathbb{Q}=H_{1}(G ; \mathbb{Q})$.

Assume now that $b_{1}(G)<\infty$. In this case, we may define the rational LCS ranks by $\phi_{n}^{\mathrm{Q}}(G):=\operatorname{dim}_{\mathbb{Q}} \operatorname{gr}_{n}^{\mathrm{Q}}(G) \otimes \mathbb{Q}$ and the rational Chen ranks by $\theta_{n}^{\mathrm{Q}}(G):=\phi_{n}^{\mathrm{Q}}\left(G / G_{\mathbb{Q}}^{\prime \prime}\right)$. With this setup, Proposition 5.1, part (2) has the following immediate corollary.

Corollary 5.2. Suppose $b_{1}(G)<\infty$. Then $\phi_{n}^{\complement}(G)=\phi_{n}(G)$ and $\theta_{n}^{\complement}(G)=\theta_{n}(G)$ for all $n \geqslant 1$.

5.3. The mod $p$ lower central series. Now fix a prime $p$. The mod-p lower central series, denoted $\left\{\gamma_{n}^{p}(G)\right\}_{n \geqslant 1}$, was introduced by Stallings in [59], and further studied in many works, including $[5,13,36,54,66]$. The series is defined inductively, as follows:

$$
\gamma_{1}^{p}(G)=G \text { and } \gamma_{n+1}^{p}(G)=\left\langle\left(\gamma_{n}^{p}(G)\right)^{p},\left[G, \gamma_{n}^{p}(G)\right]\right\rangle .
$$

This is a $p$-torsion series, meaning that $\left(\gamma_{n}^{p}(G)\right)^{p} \subseteq \gamma_{n+1}^{p}(G)$ for all $n \geqslant 1$; it is also an N-series, cf. [54]. Therefore, the successive quotients, $\operatorname{gr}_{n}^{p}(G):=\gamma_{n}^{p}(G) / \gamma_{n+1}^{p}(G)$, are $p$-torsion abelian groups, and thus can be viewed as $\mathbb{Z}_{p}$-vector spaces; in particular, $G / \gamma_{2}^{p}(G)=H_{1}\left(G ; \mathbb{Z}_{p}\right)$. The mod- $p$ LCS is the fastest-descending central series whose successive quotients are $\mathbb{Z}_{p}$-vector spaces. Clearly, the terms of the series are fully invariant subgroups.

As noted in [36], each term $G_{p}^{(r)}$ of the derived $p$-series contains $\gamma_{n}^{p}(G)$ as a normal subgroup, for all $n$ sufficiently large. The reason for this is that the $p$-lower central series of any finite $p$-group (in particular, the quotient $G / G_{p}^{(r)}$ ), terminates at 1 . Moreover, $G_{p}^{\prime}=\gamma_{2}^{p}(G)$ and $G_{p}^{\prime \prime} \subseteq \gamma_{3}^{p}(G)$. Consequently, we have a surjective homomorphism from the mod- $p$ Alexander invariant, $B_{p}(G)=G_{p}^{\prime} / G_{p}^{\prime \prime}$, to $\operatorname{gr}_{2}^{p}(G)=\gamma_{2}^{p}(G) / \gamma_{3}^{p}(G)$.

Example 5.3. If $G$ is abelian, then $\gamma_{n}^{p}(G)=G_{p}^{(n-1)}=G^{p^{n-1}}$. For instance, if $G=\mathbb{Z}=$ $\langle a\rangle$, then $\gamma_{n}^{p}(G)=\left\langle a^{p^{n-1}}\right\rangle \cong \mathbb{Z}$ for all $n \geqslant 1$. On the other hand, if $G=\mathbb{Z}_{p}^{s}$ is an elementary $p$-abelian group, then $\gamma_{n}^{p}(G)=\{1\}$ for all $n \geqslant 2$.

The associated graded Lie algebra, $\operatorname{gr}^{p}(G):=\bigoplus_{n \geqslant 1} \operatorname{gr}_{n}^{p}(G)$, with addition and Lie bracket defined as before, can be viewed as a Lie algebra over the field $\mathbb{Z}_{p}$. The assignment $G \leadsto \operatorname{gr}^{p}(G)$ is functorial. The Lie algebra $\operatorname{gr}^{p}(G)$ supports additional operations, $\operatorname{gr}_{n}^{p}(G) \rightarrow \operatorname{gr}_{n+1}^{p}(G)$, which are induced by the $p$-th power map, $g \mapsto g^{p}$. As observed in $[4, \S 12]$, this Lie algebra is generated through Lie brackets and power operations by its degree 1 piece, $\operatorname{gr}_{1}^{p}(G)=H_{1}\left(G ; \mathbb{Z}_{p}\right)$.

Assume now that $b_{1}^{p}(G)=\operatorname{dim}_{\mathbb{Z}_{p}} H_{1}\left(G ; \mathbb{Z}_{p}\right)$ is finite. By the observation we just made, all homogeneous pieces of $\operatorname{gr}^{p}(G)$ are also finite-dimensional. Hence, we may define the mod- $p$ LCS ranks of $G$ by setting $\phi_{n}^{p}(G):=\operatorname{dim}_{\mathbb{Z}_{p}} \operatorname{gr}_{n}^{p}(G)$ and the mod- $p$ Chen ranks as $\theta_{n}^{p}(G):=\operatorname{dim}_{\mathbb{Z}_{p}} \operatorname{gr}_{n}^{p}\left(G / G_{p}^{\prime \prime}\right)$. Clearly, $\phi_{n}^{p}(G) \geqslant \theta_{n}^{p}(G)$ for all $n \geqslant 1$.

Finally, suppose $G$ is finitely generated; a previous remark then gives $\operatorname{dim}_{\mathbb{Z}_{p}} B_{p}(G) \geqslant$ $\phi_{2}^{p}(G)$. If $b_{2}^{p}(G)$ is also finite, work of Shalen and Wagreich [58] (see also Lackenby 
[35]) shows that $\phi_{2}^{p}(G) \geqslant\left(\begin{array}{c}b_{1}^{p}(G) \\ 2\end{array}\right)+b_{1}^{p}(G)-b_{2}^{p}(G)$. Putting these inequalities together yields a lower bound on the dimension of the $\mathbb{Z}_{p}$-vector space $B_{p}(G)$, solely in terms of the first two mod- $p$ Betti numbers of $G$.

Example 5.4. If $G$ is the fundamental group of a closed 3-manifold (assumed to be orientable if $p$ is odd), then Poincaré duality gives $\operatorname{dim}_{\mathbb{Z}_{p}} B_{p}(G) \geqslant\left(\begin{array}{c}b_{1}^{p}(G) \\ 2\end{array}\right)$. For $G=$ $\mathbb{Z}^{3}$ this is an equality, but in general the inequality is strict. For instance, if $G$ is the Heisenberg nilmanifold group of $3 \times 3$ upper diagonal integral matrices with 1 's down the diagonal, then $b_{1}^{p}(G)=2$, yet $B_{p}(G)=\mathbb{Z}_{p} \oplus \mathbb{Z}_{p}$.

\section{Part II. Group extensions}

\section{MASSEY'S CORRESPONDENCE}

We now switch our focus to group extensions. After a brief discussion of the monodromy action of an extension $1 \rightarrow K \rightarrow G \rightarrow Q \rightarrow 1$, we present a detailed overview of Massey's correspondence between the filtration of the Alexander invariant of $K$ by powers of the augmentation ideal of $Q$ and the lower central series of the maximal metabelian quotient of $G$. Finally, we extend this correspondence to the rational and mod- $p$ settings.

6.1. Monodromy action. We start with a quick review of group extensions; for more on this topic, we refer to [8, Ch. IV] and [33, Ch. VI]. Consider a short exact sequence of groups,

$$
1 \longrightarrow K \stackrel{\iota}{\longrightarrow} G \stackrel{\pi}{\longrightarrow} Q \longrightarrow 1 .
$$

Let $\operatorname{Aut}(K)$ be the group of right automorphisms of $K$, with group operation $\alpha \cdot \beta=\beta \circ \alpha$. Choosing a set-theoretic section of the projection map $\pi$, i.e., a function $\sigma: Q \rightarrow G$ such that $\pi \circ \sigma=\mathrm{id}_{Q}$, defines a function $\varphi: Q \rightarrow \operatorname{Aut}(K)$ by setting $\varphi(x)(a)=\sigma(x) a \sigma(x)^{-1}$ for $x \in Q$ and $a \in K$.

In general, this function (which does depend on the choice of section) is not a homomorphism. Nevertheless, if we post-compose it with the projection from $\operatorname{Aut}(K)$ to the $\operatorname{group} \operatorname{Out}(K):=\operatorname{Aut}(K) / \operatorname{Inn}(K)$, the resulting map, $\tilde{\varphi}: Q \rightarrow \operatorname{Out}(K)$, is a group homomorphism, which does not depend on the choice of section used to define it. The map sending and automorphism of $K$ to the induced automorphism of $K_{\mathrm{ab}}$ factors through a homomorphism, $v: \operatorname{Out}(K) \rightarrow \operatorname{Aut}\left(K_{\mathrm{ab}}\right)$. We call the composite $v \circ \tilde{\varphi}: Q \rightarrow \operatorname{Aut}\left(K_{\mathrm{ab}}\right)$ the monodromy representation of the extension (38).

Assume now that the extension (38) is split exact, i.e., there is a homomorphism $\sigma: Q \rightarrow G$ such that $\pi \circ \sigma=\operatorname{id}_{Q}$. In this case, the corresponding function, $\varphi: Q \rightarrow$ $\operatorname{Aut}(K)$, is a well-defined homomorphism. This approach realizes the group $G$ as a split extension, $G=K \rtimes_{\varphi} Q$. That is, $G$ equals $K \times Q$ as a set, and its group operation can be expressed as $\left(x_{1}, y_{1}\right) \cdot\left(x_{2}, y_{2}\right)=\left(x_{1} \varphi\left(y_{1}\right)\left(x_{2}\right), y_{1} y_{2}\right)$. In what follows, we will identify the group $Q$ with its image under the splitting, $\sigma(Q) \leqslant G$, and thus view $Q$ as a subgroup of $G$; likewise, we will identify $K$ with $\iota(K)$, and thus view it as a normal 
subgroup of $G$. With these identifications, the action of $Q$ on $K$ is simply the restriction of the conjugation action in $G$; that is, $\varphi(y)(x)=y x y^{-1}$.

6.2. Massey's correspondence. Suppose now that the normal subgroup $K \triangleleft G$ from (38) is abelian. In that case, Massey established in [41] a simple, yet very fruitful connection between $B(K)$, the Alexander invariant of $K$ and the lower central series of $G / G^{\prime \prime}$, the maximal metabelian quotient of $G$. Since the original reference contains only sketches of proofs, we provide complete details, which will also serve as a blueprint for the rational and modular extensions of this correspondence that we will give below.

Since the group $K$ is assumed to be abelian, the monodromy of the extension, $\varphi=$ $v \circ \tilde{\varphi}: Q \rightarrow \operatorname{Aut}(K)$, is a well-defined homomorphism, which puts the structure of a $\mathbb{Z}[Q]$-module on $K$. For a group $G$ and a coefficient ring $\mathbb{k}$, we denote by $I_{\mathbb{k}}(G)$ the augmentation ideal of $\mathbb{k}[G]$, that is, the kernel of the ring map $\varepsilon: \mathbb{k}[G] \rightarrow \mathbb{k}$ defined by $\varepsilon\left(\sum n_{g} g\right)=\sum n_{g}$.

Lemma 6.1 ([41]). Let $1 \rightarrow K \rightarrow G \rightarrow Q \rightarrow 1$ be an extension of groups, and assume $K$ is abelian. Define a filtration $\left\{K_{n}\right\}_{n \geqslant 0}$ on $K$ inductively, by setting $K_{0}=K$ and $K_{n+1}=$ $\left[G, K_{n}\right]$, and let $I=I_{\mathbb{Z}}(Q)$. Then $K_{n}=I^{n} \cdot K$ for all $n \geqslant 0$.

Proof. We write the group operation in $K$ multiplicatively when viewing it as a subgroup of $G$, and additively when viewing it as a $\mathbb{Z}[Q]$-module. Fix a set-section $\sigma: Q \rightarrow G$ of the projection $\pi: G \rightarrow Q$, and let $\varphi: Q \rightarrow \operatorname{Aut}(K)$ be the corresponding monodromy. An element $h \in Q$ then acts on $K$ by sending an element $k \in K$ to $h \cdot k=\varphi(h)(k)=$ $g k g^{-1} \in K$, where $g=\sigma(h) \in G$.

The claim is now proved by induction on $n$, with the base case $n=0$ being obvious. So assume $K_{n}=I^{n} K$. Consider a commutator $g k^{-1} k^{-1} \in K_{n+1}=\left[G, K_{n}\right]$ with $g \in G$ and $k \in K_{n}$. In view of the above observations, such a commutator corresponds in one-to-one fashion to the element $(h-1) \cdot k \in I K_{n}$, where $h=\pi(g)$. By the induction hypothesis, $I K_{n}=I^{n+1} K$; thus, $K_{n+1}=I^{n+1} K$, and we are done.

Theorem 6.2 ([41]). Let $G$ be a group, and let $I=I_{\mathbb{Z}}\left(G_{\mathrm{ab}}\right)$. Then $I^{n} B(G)=\gamma_{n+2}\left(G / G^{\prime \prime}\right)$, for all $n \geqslant 0$.

Proof. Consider the extension $1 \rightarrow G^{\prime} / G^{\prime \prime} \rightarrow G / G^{\prime \prime} \rightarrow G / G^{\prime} \rightarrow 1$ from (7), and recall that the Alexander invariant $B(G)$ is the (abelian) group $G^{\prime} / G^{\prime \prime}$, viewed as a module over $\mathbb{Z}\left[G_{\text {ab }}\right]$. Let $\left\{\left(G^{\prime} / G^{\prime \prime}\right)_{n}\right\}_{n \geqslant 0}$ be the filtration of the subgroup $K=G^{\prime} / G^{\prime \prime}$ defined in Lemma 6.1 ; the lemma then gives $\left(G^{\prime} / G^{\prime \prime}\right)_{n}=I^{n} B(G)$ for all $n \geqslant 0$.

It remains to show that $\left(G^{\prime} / G^{\prime \prime}\right)_{n}=\gamma_{n+2}\left(G / G^{\prime \prime}\right)$ for all $n \geqslant 0$. (Note that $\gamma_{n+2}\left(G / G^{\prime \prime}\right)$ is a subgroup of $\gamma_{2}\left(G / G^{\prime \prime}\right)=G^{\prime} / G^{\prime \prime}$, and thus is an abelian group.) We prove this claim by induction, with the base case $n=0$ clearly holding. For the induction step, we have that $\left(G^{\prime} / G^{\prime \prime}\right)_{n+1}=\left[G,\left(G^{\prime} / G^{\prime \prime}\right)_{n}\right]=\left[G, \gamma_{n+2}\left(G / G^{\prime \prime}\right)\right]=\gamma_{n+3}\left(G / G^{\prime \prime}\right)$, and the proof is complete. 
6.3. Completion and associated graded of $B(G)$. Recall that the $\operatorname{ring} R=\mathbb{Z}\left[G_{\mathrm{ab}}\right]$ admits a filtration by powers of the augmentation ideal $I=I_{\mathbb{Z}}\left(G_{\mathrm{ab}}\right)$. Let $\widehat{R}=\lim R / I^{n}$ be the completion of $R$ with respect to this filtration, and let $\operatorname{gr}(R)=\operatorname{gr}(\widehat{R})=\bigoplus_{n \geqslant 0} I^{n} / I^{n+1}$ be the associated graded object. Both $\widehat{R}$ and $\operatorname{gr}(R)$ acquire in a natural way a ring structure, which is compatible with the filtration, respectively, the grading.

Let $\widehat{B}=\lim B / I^{n} B$ be the $I$-adic completion of the Alexander invariant $B=B(G)$, viewed as a module over $\widehat{R}$, and let $\operatorname{gr}(\widehat{B})=\operatorname{gr}(B)$ be the associated graded object, viewed as a (graded) module over $\operatorname{gr}(R)$. As such, $\operatorname{gr}(B)$ is generated by its degree 0 piece, $B / I B$. It follows from Theorem 6.2 that the $\operatorname{gr}(R)$-generators of $\operatorname{gr}(B)$ correspond to a generating set for $\operatorname{gr}_{2}(G)$; moreover, the theorem has the following immediate corollary.

Corollary 6.3 ([41]). $\operatorname{gr}_{n}(B(G)) \cong \operatorname{gr}_{n+2}\left(G / G^{\prime \prime}\right)$, for all $n \geqslant 0$.

Now suppose that $b_{1}(G)<\infty$. Then $\operatorname{gr}(B(G) \otimes \mathbb{Q})$ is a finitely generated graded module over the graded ring $\operatorname{gr}\left(\mathbb{Q}\left[G_{\mathrm{ab}}\right]\right)$. Let $\theta_{n}(G)=\operatorname{dim}_{\mathbb{Q}} \operatorname{gr}\left(G / G^{\prime \prime}\right) \otimes \mathbb{Q}$ be the Chen ranks of $G$, starting with $\theta_{1}(G)=b_{1}(G)$. As a consequence of Corollary 6.3, the Hilbert series of the rationalization of $\operatorname{gr}(B(G))$ determines the Chen ranks of $G$, as follows,

$$
\operatorname{Hilb}(\operatorname{gr}(B(G) \otimes \mathbb{Q}), t)=\sum_{n \geqslant 0} \theta_{n+2}(G) t^{n} .
$$

Let $\alpha: G \rightarrow H$ be a group homomorphism. Recall from $\S 2.4$ that $\alpha$ induces a map of modules, $B(\alpha): B(G) \rightarrow B(H)$, which covers the ring map $\tilde{\alpha}_{\mathrm{ab}}: R \rightarrow S$, where $S=\mathbb{Z}\left[H_{\mathrm{ab}}\right]$. Clearly, the map $B(\alpha)$ preserves $I$-adic filtrations, and thus induces a morphism $\widehat{B(\alpha)}: \widehat{B(G)} \rightarrow \widehat{B(H)}$ which covers the ring map $\hat{\tilde{\alpha}}_{\mathrm{ab}}: \widehat{R} \rightarrow \widehat{S}$. Passing to associated graded objects, we obtain the morphism $\operatorname{gr}(B(\alpha)): \operatorname{gr}(B(G)) \rightarrow \operatorname{gr}(B(H))$, which covers the ring map $\operatorname{gr}\left(\tilde{\alpha}_{\mathrm{ab}}\right): \operatorname{gr}(R) \rightarrow \operatorname{gr}(S)$. For future reference, we record a fact regarding this last map.

Lemma 6.4. Suppose $H_{\mathrm{ab}}$ is finitely generated and the map $\alpha_{\mathrm{ab}}: G_{\mathrm{ab}} \rightarrow H_{\mathrm{ab}}$ is injective. Then the map $\operatorname{gr}\left(\tilde{\alpha}_{\mathrm{ab}}\right): \operatorname{gr}(R) \rightarrow \operatorname{gr}(S)$ is also injective.

Proof. Our assumptions imply that $G_{\mathrm{ab}}$ is also finitely generated. Letting $r$ and $s$ denote the minimum number of generators of $G_{\mathrm{ab}}$ and $H_{\mathrm{ab}}$, respectively, the map $\alpha_{\mathrm{ab}}$ lifts to an injective $\mathbb{Z}$-linear map, $\mathbb{Z}^{r} \rightarrow \mathbb{Z}^{s}$, given by multiplication with a matrix $M$.

The ring $\operatorname{gr}(R)$ can be described as the quotient of the polynomial ring $\mathbb{Z}\left[x_{1}, \ldots, x_{r}\right]$ by a monomial ideal determined by $\operatorname{Tors}\left(G_{\mathrm{ab}}\right)$, and likewise for $\operatorname{gr}(S)$. The ring map $\operatorname{gr}\left(\tilde{\alpha}_{\mathrm{ab}}\right): \operatorname{gr}(R) \rightarrow \operatorname{gr}(S)$ lifts to a map between polynomial rings, $\mu: \mathbb{Z}\left[x_{1}, \ldots, x_{r}\right] \rightarrow$ $\mathbb{Z}\left[y_{1}, \ldots, y_{s}\right]$, which may be identified with the linear change of variables defined by $M$. Clearly, the map $\mu$ is injective, and thus the map $\operatorname{gr}\left(\tilde{\alpha}_{\mathrm{ab}}\right)$ is also injective.

6.4. A rational Massey correspondence. The next two results are rational analogues of Massey's correspondence. Both the statements and the proofs are similar to the integral case, though they do require some modifications, which we record below. 
Lemma 6.5. Let $1 \rightarrow K \rightarrow G \rightarrow Q \rightarrow 1$ be an extension of groups, and assume $K$ is abelian. Define a filtration $\left\{K_{n}^{\mathrm{Q}}\right\}_{n \geqslant 0}$ on $K$ inductively, by setting $K_{0}^{\mathrm{Q}}=K$ and $K_{n+1}^{\mathbb{Q}}=\sqrt{\left[G, K_{n}^{\mathbb{Q}}\right]}$, and let $I=I_{\mathbb{Q}}(Q)$. Then $K_{n}^{\mathbb{Q}} \otimes \mathbb{Q}=I^{n} \cdot(K \otimes \mathbb{Q})$ for all $n \geqslant 0$.

Proof. Recall that the action of $h \in Q$ on $k \in K$ is given by $h \cdot k=\mathrm{gkg}^{-1}$, where $g=\sigma(h) \in G$. By definition, an element $x \in K$ belongs to $\sqrt{[G, K]}$ if there is an integer $m>0$ such that $x^{m}$ can be written as a product of commutators of the form $g k g^{-1} k^{-1} \in[G, K]$. Viewing now the $\mathbb{Q}$-vector space $\sqrt{[G, K]} \otimes \mathbb{Q}$ as a module over $\mathbb{Q}[Q]$, the element $x$ corresponds to a sum of elements of the form $\frac{1}{m}(h-1) \cdot k \in I \cdot(K \otimes \mathbb{Q})$. This shows that $\sqrt{[G, K]} \otimes \mathbb{Q}=I \cdot(K \otimes \mathbb{Q})$, proving the claim for $n=1$. The general case follows by induction on $n$, as in the proof of Lemma 6.1.

Theorem 6.6. Let $G$ be a group and let $I=I_{\mathbb{Q}}\left(G_{\mathrm{abf}}\right)$. Then $I^{n}\left(B_{\mathbb{Q}}(G) \otimes \mathbb{Q}\right)=\gamma_{n+2}^{\mathbb{Q}}\left(G / G_{\mathbb{Q}}^{\prime \prime}\right) \otimes$ $\mathbb{Q}$, for all $n \geqslant 0$.

Proof. Consider the extension $1 \rightarrow G_{\odot}^{\prime} / G_{\odot}^{\prime \prime} \rightarrow G / G_{\odot}^{\prime \prime} \rightarrow G / G_{\odot}^{\prime} \rightarrow 1$ from (19), and recall that the rational Alexander invariant $B_{\mathrm{Q}}(G)$ is equal to $G_{\mathrm{Q}}^{\prime} / G_{\mathrm{Q}}^{\prime \prime}$, viewed as a module over $\mathbb{Z}\left[G_{\text {abf }}\right]$. Let $\left\{\left(G_{\odot}^{\prime} / G_{\mathrm{Q}}^{\prime \prime}\right)_{n}^{Q}\right\}_{n \geqslant 0}$ be the filtration on $G_{\mathrm{Q}}^{\prime} / G_{\mathrm{Q}}^{\prime \prime}$ defined in Lemma 6.5; the lemma then gives $\left(G_{\mathrm{Q}}^{\prime} / G_{\mathrm{Q}}^{\prime \prime}\right)_{n}^{\mathrm{Q}} \otimes \mathbb{Q}=I^{n} \cdot\left(B_{\mathbb{Q}}(G) \otimes \mathbb{Q}\right)$ for all $n \geqslant 0$.

To complete the proof, we show by induction that $\left(G_{\mathrm{Q}}^{\prime} / G_{\mathrm{Q}}^{\prime \prime}\right)_{n}^{\varrho}=\gamma_{n+2}^{Q}\left(G / G_{\mathrm{Q}}^{\prime \prime}\right)$ for all $n \geqslant 0$. (Note that $\gamma_{n+2}^{\mathrm{Q}}\left(G / G_{\mathrm{Q}}^{\prime \prime}\right)$ is a subgroup of $\gamma_{2}^{\mathrm{Q}}\left(G / G_{\mathrm{Q}}^{\prime \prime}\right)=G_{\mathrm{Q}}^{\prime} / G_{\mathrm{Q}}^{\prime \prime}$, and thus is a torsion-free abelian group.) The base case $n=0$ is immediate. For the induction step, we have that

$$
\left(G_{\mathrm{Q}}^{\prime} / G_{\mathrm{Q}}^{\prime \prime}\right)_{n+1}^{\mathrm{Q}}=\sqrt{\left[G,\left(G_{\mathrm{Q}}^{\prime} / G_{\mathrm{Q}}^{\prime \prime}\right)_{n}^{\mathrm{Q}}\right]}=\sqrt{\left[G, \gamma_{n+2}^{\mathrm{Q}}\left(G / G_{\mathrm{Q}}^{\prime \prime}\right)\right]}=\gamma_{n+3}^{\mathrm{Q}}\left(G / G_{\mathrm{Q}}^{\prime \prime}\right),
$$

and we are done.

Corollary 6.7. $\operatorname{gr}_{n}\left(B_{\mathbb{Q}}(G) \otimes \mathbb{Q}\right) \cong \operatorname{gr}_{n+2}\left(G / G_{\mathbb{Q}}^{\prime \prime}\right) \otimes \mathbb{Q}$, for all $n \geqslant 0$.

Proof. Follows from Theorem 6.6 by taking associated graded groups with respect to the $I$-adic filtration on $B_{\mathbb{Q}}(G) \otimes \mathbb{Q}$ and the $\gamma^{\mathbb{Q}}\left(G / G_{\mathbb{Q}}^{\prime \prime}\right)$ filtration on $G / G_{\mathbb{Q}}^{\prime \prime}$, respectively.

Corollary 6.8. If $b_{1}(G)<\infty$, then, for all $n \geqslant 2$,

$$
\theta_{n}(G)=\theta_{n}^{\mathrm{Q}}(G)=\operatorname{dim}_{\mathbb{Q}} \operatorname{gr}_{n-2}(B(G) \otimes \mathbb{Q})=\operatorname{dim}_{\mathbb{Q}} \operatorname{gr}_{n-2}\left(B_{\mathbb{Q}}(G) \otimes \mathbb{Q}\right) .
$$

Proof. The first equality follows from Corollary 5.2. By formula (39) we have $\theta_{n}(G)=$ $\operatorname{dim}_{\mathbb{Q}} \operatorname{gr}_{n-2}(B(G) \otimes \mathbb{Q})$, while by Corollary 6.7 we have $\theta_{n}^{\mathbb{Q}}(G)=\operatorname{dim}_{\mathbb{Q}} \operatorname{gr}_{n-2}\left(B_{\mathbb{Q}}(G) \otimes \mathbb{Q}\right)$ for all $n \geqslant 2$.

6.5. An isomorphism between completions. Set $R_{0}=\mathbb{Z}\left[G_{\text {abf }}\right]$ and $I_{0}=I_{\mathbb{Z}}\left(G_{\text {abf }}\right)$. Let $\widehat{R_{0}}$ be the completion of $R_{0}$ with respect to the $I_{0}$-adic filtration, and let $\widehat{B_{\mathrm{Q}}}$ be the $I_{0}$-adic completion of the rational Alexander invariant $B_{Q}=B_{Q}(G)$, viewed as a module over $\widehat{R_{0}}$.

The projection map $v: G_{\mathrm{ab}} \rightarrow G_{\mathrm{abf}}$ induces a surjective ring map, $\tilde{v}: R \rightarrow R_{0}$. By Proposition 3.5, the inclusion $G^{\prime} \hookrightarrow G_{\odot}^{\prime}$ induces a $\tilde{v}$-morphism, $\kappa: B \rightarrow B_{Q}$, which becomes a surjection upon tensoring with $\mathbb{Q}$, but not necessarily an isomorphism. The next 
proposition shows that, upon completion, the map $\kappa \otimes \mathbb{Q}$ does become an isomorphism. This result overlaps with [20, Proposition 2.4], which is both slightly more general (in that it replaces $G_{\mathrm{Q}}^{\prime}$ with an arbitrary subgroup $H \leqslant G$ containing $G^{\prime}$ as a finite-index normal subgroup), and less general (in that it assumes $G$ to be finitely generated, which we don't). Our proof, though, is very much different.

Theorem 6.9. Let $G$ be a group with $b_{1}(G)<\infty$. Then the map $\kappa: B(G) \rightarrow B_{\mathbb{Q}}(G)$ yields

(1) An isomorphism $\hat{\kappa} \otimes \mathbb{Q}: B \widehat{B(G) \otimes \mathbb{Q}} \stackrel{\simeq}{\longrightarrow} B_{\mathbb{Q}} \widehat{(G) \otimes} \mathbb{Q}$ of filtered modules covering the filtered ring map $\widehat{\tilde{v}} \otimes \mathbb{Q}: \widehat{R} \otimes \mathbb{Q} \rightarrow \widehat{R_{0}} \otimes \mathbb{Q}$.

(2) An isomorphism $\operatorname{gr}(\kappa) \otimes \mathbb{Q}: \operatorname{gr}(B(G) \otimes \mathbb{Q}) \stackrel{\simeq}{\longrightarrow} \operatorname{gr}\left(B_{\mathbb{Q}}(G) \otimes \mathbb{Q}\right)$ covering the map of graded rings $\operatorname{gr}(\tilde{v}): \operatorname{gr}(R) \otimes \mathbb{Q} \rightarrow \operatorname{gr}\left(R_{0}\right) \otimes \mathbb{Q}$.

Proof. The ring epimorphism $\tilde{v}: R \rightarrow R_{0}$ preserves augmentation ideals; therefore, it induces a filtration-preserving ring map, $\widehat{\tilde{v}}: \widehat{R} \rightarrow \widehat{R}_{0}$. Likewise, the map $\kappa: B \rightarrow B_{\mathrm{Q}}$ is compatible with the $I$-adic and $I_{0}$-adic filtrations in source and target, and thus induces a filtration-preserving $\widehat{\tilde{v}}$-morphism on completions, $\hat{\kappa}: \widehat{B} \rightarrow \widehat{B_{\mathbb{Q}}}$.

This morphism induces a morphism between the corresponding associated graded modules, $\operatorname{gr}(\kappa): \operatorname{gr}(B) \rightarrow \operatorname{gr}\left(B_{\odot}\right)$, covering the ring map $\operatorname{gr}(\tilde{v}): \operatorname{gr}(R) \rightarrow \operatorname{gr}\left(R_{0}\right)$. On the other hand, we have by Proposition 5.1 a morphism of graded Lie algebras, $\Phi: \operatorname{gr}(G) \rightarrow$ $\operatorname{gr}^{\mathbb{Q}}(G)$, which induces an isomorphism $\Phi \otimes \mathbb{Q}: \operatorname{gr}(G) \otimes \mathbb{Q} \rightarrow \operatorname{gr}^{\complement}(G) \otimes \mathbb{Q}$.

For each $n \geqslant 0$ we have a commuting diagram,

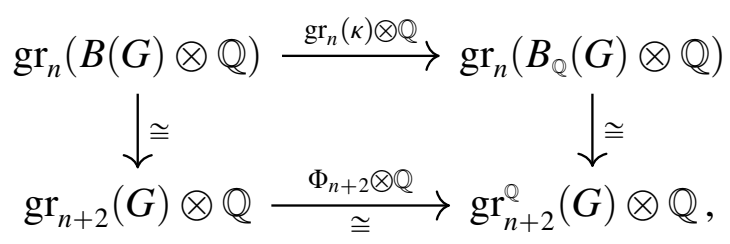

where the vertical arrows are the isomorphisms provided by Corollaries 6.3 and 6.7. It follows that the top arrow is an isomorphism, for each $n \geqslant 0$, Hence, the map $\operatorname{gr}(\kappa) \otimes$ $\mathbb{Q}: \operatorname{gr}(B \otimes \mathbb{Q}) \rightarrow \operatorname{gr}\left(B_{\mathbb{Q}} \otimes \mathbb{Q}\right)$ is an isomorphism. A standard argument (see e.g. [72, Lemma 2.4]) now implies that the map $\hat{\kappa} \otimes \mathbb{Q}: \widehat{B \otimes \mathbb{Q}} \rightarrow \widehat{B_{\mathbb{Q}} \otimes \mathbb{Q}}$ is an isomorphism, too.

Remark 6.10. Setting $r=b_{1}(G)$ and picking a generating set $t_{1}, \ldots, t_{r}$ for $G_{\text {abf }}$ allows us to identify the ring $\mathbb{Z}\left[G_{\text {abf }}\right]$ with the ring of Laurent polynomials $\mathbb{Z}\left[t_{1}^{ \pm 1}, \ldots, t_{r}^{ \pm 1}\right]$ and the $\operatorname{ring} \operatorname{gr}\left(\mathbb{Z}\left[G_{\mathrm{abf}}\right]\right)$ with the polynomial ring $\mathbb{Z}\left[x_{1}, \ldots, x_{r}\right]$, where $x_{i}$ corresponds to $t_{i}-1$. This permits direct computation of the Hilbert series of $\operatorname{gr}(B(G) \otimes \mathbb{Q}) \cong \operatorname{gr}\left(B_{\mathbb{Q}}(G) \otimes \mathbb{Q}\right)$, and thus of the Chen ranks $\theta_{n}(G)=\theta_{n}^{\mathrm{e}}(G)$, too, via standard methods of commutative algebra, based on the use of Gröbner bases. We refer to [17, 61, 16, 71] for detailed explanations and examples on how this algorithm works in various settings. 
6.6. A modular Massey correspondence. For the rest of this section, we fix a prime $p$. The next two results are mod- $p$ analogues of Massey's correspondence.

Lemma 6.11. Let $1 \rightarrow K \rightarrow G \rightarrow Q \rightarrow 1$ be an extension of groups, and assume $K$ is an elementary abelian p-group. Define a filtration $\left\{K_{n}\right\}_{n \geqslant 0}$ on $K$ inductively, by setting $K_{0}=K$ and $K_{n+1}=\left[G, K_{n}\right]$, and let $I=I_{\mathbb{Z}_{p}}\left(H_{1}\left(Q ; \mathbb{Z}_{p}\right)\right)$. Then $K_{n}=I^{n} \cdot K$ for all $n \geqslant 0$.

Proof. By assumption, $K$ is the underlying additive group of a $\mathbb{Z}_{p}$-vector space. Thus, the monodromy action of the extension defines the structure of a $\mathbb{Z}_{p}\left[H_{1}\left(Q ; \mathbb{Z}_{p}\right)\right]$-module on $K$. The proof now proceeds as in the proof for Lemma 6.1.

Theorem 6.12. Let $G$ be a group, and let $I=I_{\mathbb{Z}_{p}}\left(H_{1}\left(G ; \mathbb{Z}_{p}\right)\right)$. Then $I^{n} B_{p}(G)=\gamma_{n+2}^{p}\left(G / G_{p}^{\prime \prime}\right)$, for all $n \geqslant 0$.

Proof. Consider the extension $1 \rightarrow G_{p}^{\prime} / G_{p}^{\prime \prime} \rightarrow G / G_{p}^{\prime \prime} \rightarrow G / G_{p}^{\prime} \rightarrow 1$ from (26), and recall that the mod- $p$ Alexander invariant $B_{p}(G)$ is equal to $G_{p}^{\prime} / G_{p}^{\prime \prime}$, an elementary abelian $p$-group. Using now Lemma 6.11, the conclusion follows as in the proof of Theorem 6.2 .

Finally, assume that $\operatorname{dim}_{\mathbb{Z}_{p}} H_{1}\left(G ; \mathbb{Z}_{p}\right)$ is finite, and let $\theta_{n}^{p}(G)=\operatorname{dim}_{\mathbb{Z}_{p}} \operatorname{gr}_{n}^{p}\left(G / G_{p}^{\prime \prime}\right)$ be the mod- $p$ Chen ranks of $G$. Also let $S=\operatorname{gr}\left(\mathbb{Z}_{p}\left[H_{1}\left(G ; \mathbb{Z}_{p}\right)\right]\right)$ be the associated graded ring with respect to the $I$-adic filtration on $\mathbb{Z}_{p}\left[H_{1}\left(G ; \mathbb{Z}_{p}\right)\right]$, and let $\operatorname{gr}\left(B_{p}(G)\right)$ be the associated graded $S$-module with respect to the $I$-adic filtration on $B_{p}(G)$. As a corollary to Theorem 6.12, we obtain the following formula relating the Hilbert series of this graded module to the generating series for the mod- $p$ Chen ranks of $G$ :

$$
\operatorname{Hilb}\left(\operatorname{gr}\left(B_{p}(G)\right), t\right)=\sum_{n \geqslant 0} \theta_{n+2}^{p}(G) t^{n} .
$$

\section{SPLIT EXTENSIONS AND LOWER CENTRAL SERIES}

In this section we restrict our attention to split extensions, $G=K \rtimes_{\varphi} Q$, and discuss the relationship between the lower central series and the associated graded Lie algebras of the factors and those of the extension.

7.1. A generalized Falk-Randell theorem. We start with a recent result of Guaschi and Pereira [30], which expresses the lower central series of a split extension in terms of the lower central series of the factors, as well as the extension data.

Theorem 7.1 ([30]). Let $G=K \rtimes_{\varphi} Q$ be a split extension of groups. For each $n \geqslant 1$ there is a split extension, $\gamma_{n}(G)=L_{n} \rtimes_{\varphi} \gamma_{n}(Q)$, where $L_{1}=K$ and $L_{n} \leqslant K$ is the subgroup generated by $\left[K, L_{n-1}\right]$, $\left[K, \gamma_{n-1}(Q)\right]$, and $\left[L_{n-1}, Q\right]$.

In [66] we give a more streamlined proof of this theorem, which exploits the fact (proved there) that the series $L=\left\{L_{n}\right\}_{n \geqslant 1}$ is an N-series for the group $K$.

Following Falk and Randell [29], we say that a split extension $G=K \rtimes_{\varphi} Q$ is an almost direct product if $Q$ acts trivially on $K_{\mathrm{ab}}=H_{1}(K ; \mathbb{Z})$. This condition is equivalent 
to $\varphi(x)(a) \cdot a^{-1} \in K^{\prime}$, for all $x \in Q$ and $a \in K$. Viewing $K$ and $Q$ as subgroups of $G$ as explained in $\S 6.1$, the condition can be written as $[K, Q] \subseteq \gamma_{2}(K)$. As shown in [6, Proposition 6.3], the property of being an almost direct product does not depend on the choice of a splitting for the extension.

When $Q$ acts trivially on $K_{\mathrm{ab}}$, we prove in [66] that $L_{n}=\gamma_{n}(K)$. In view of Theorem 7.1, this recovers the following well-known result of Falk and Randell [29].

Theorem 7.2 ([29]). Suppose $G=K \rtimes_{\varphi} Q$ is an almost direct product of groups. Then,

(1) $\gamma_{n}(G)=\gamma_{n}(K) \rtimes_{\varphi} \gamma_{n}(Q)$, for all $n \geqslant 1$.

(2) $\operatorname{gr}(G)=\operatorname{gr}(K) \rtimes_{\bar{\varphi}} \operatorname{gr}(Q)$.

Under additional assumptions, we have the following corollary, which will be needed in the proof of Theorem 8.7.

Corollary 7.3. Let $G=K \rtimes_{\varphi} Q$ be an almost direct product, and assume $Q$ is abelian. Then,

(1) $\gamma_{n}(K)=\gamma_{n}(G)$ for all $n \geqslant 2$ and $\operatorname{gr}_{\geqslant 2}(K)=\operatorname{gr}_{\geqslant 2}(G)$.

(2) If, moreover, $b_{1}(G)<\infty$, then $\phi_{n}(K)=\phi_{n}(G)$ for all $n \geqslant 2$.

Proof. Since $Q$ is abelian, we have that $\gamma_{n}(K)=\{1\}$ for all $n \geqslant 2$. Thus, $\operatorname{gr}_{1}(Q)=Q$ and $\operatorname{gr}_{\geqslant 2}(Q)=0$. The first claim now follows from Theorem 7.2.

By part $(1)$, we have that $\operatorname{gr}_{n}(K) \otimes \mathbb{Q} \cong \operatorname{gr}_{n}(G) \otimes \mathbb{Q}$ for $n \geqslant 2$. Since $b_{1}(G)<\infty$, the discussion from $§ 5.1$ shows that all these vector spaces are finite-dimensional. The second claim now follows from the definition (33) of the LCS ranks.

7.2. A rational Falk-Randell theorem. Returning to the general case of a split extension, $G=K \rtimes_{\varphi} Q$, we exploit in [66] the fact that the sequence of subgroups $L=\left\{L_{n}\right\}_{n \geqslant 1}$ defined above is an N-series for $K$ in order to show (using [42]) that the series $\sqrt{L}=\left\{\sqrt{L_{n}}\right\}_{n \geqslant 1}$ is also an N-series for $K$. Building on this observation, and adapting the method of proof of Theorems 7.1 and 7.2 to this situation, we establish in [66] rational versions of the aforementioned results, as follows.

Theorem 7.4 ([66]). Let $G=K \rtimes_{\varphi} Q$ be a split extension of groups. For each $n \geqslant 1$, there is then a split extension, $\gamma_{n}^{\mathrm{Q}}(G)=\sqrt{L_{n}} \rtimes_{\varphi} \gamma_{n}^{\mathrm{Q}}(Q)$.

Theorem 7.5 ([66]). Let $G=K \rtimes_{\varphi} Q$ be a split extension, and assume $Q$ acts trivially on $K_{\mathrm{abf}}$. Then $\sqrt{L}=\gamma^{\mathrm{Q}}(K)$, and

(1) $\gamma_{n}^{\mathrm{Q}}(G)=\gamma_{n}^{\mathrm{Q}}(K) \rtimes_{\varphi} \gamma_{n}^{\mathrm{Q}}(Q)$, for all $n \geqslant 1$.

(2) $\operatorname{gr}^{\complement}(G)=\operatorname{gr}^{\complement}(K) \rtimes_{\bar{\varphi}} \operatorname{gr}^{\complement}(Q)$.

Under additional assumptions, we have the following corollary, which will be needed in the proof of Theorem 9.8.

Corollary 7.6. Let $G=K \rtimes_{\varphi} Q$ be a split extension. Assume $Q$ is torsion-free abelian and acts trivially on $K_{\mathrm{abf}}$. Then, 
(1) $\gamma_{n}^{\mathrm{e}}(K)=\gamma_{n}^{\mathrm{e}}(G)$ for all $n \geqslant 2$ and $\mathrm{gr}_{\geqslant 2}^{\mathrm{Q}}(K)=\operatorname{gr}_{\geqslant 2}^{\mathrm{Q}}(G)$.

(2) If, moreover, $b_{1}(G)<\infty$, then $\phi_{n}(K)=\phi_{n}(G)$ for all $n \geqslant 2$.

Proof. Since $Q$ is torsion-free abelian, $\gamma_{2}^{\mathrm{Q}}(Q)=\{1\}$, and so $\gamma_{n}^{\mathrm{Q}}(Q)=\{1\}$ for all $n \geqslant 2$. The first claim now follows from Theorem 7.5.

Next, assume that $b_{1}(G)<\infty$. Then, by the discussion in $\$ 5.2$, all the graded pieces of $\operatorname{gr}^{\mathrm{Q}}(G) \otimes \mathbb{Q}$ are finite-dimensional. Moreover, by Corollary 5.2, $\phi_{n}(G)=\phi_{n}^{\mathrm{Q}}(G)=$ $\operatorname{dim}_{\mathbb{Q}} \operatorname{gr}_{n}^{\mathrm{Q}}(G) \otimes \mathbb{Q}$, and the second claim now follows from the first one.

7.3. A mod- $p$ Falk-Randell theorem. Finally, we also prove in [66] mod- $p$ versions of the above theorems, recovering in the process a result of Bellingeri and Gervais from [5]. Given a split extension of groups, $G=K \rtimes_{\varphi} Q$, and a prime $p$, we define a sequence of subgroups of $K$, denoted $\left\{L_{n}^{\mathfrak{p}}\right\}_{n \geqslant 1}$, by setting $L_{1}^{\mathfrak{p}}=K$ and letting

$$
L_{n+1}^{\mathfrak{p}}=\left\langle\left(L_{n}^{\mathfrak{p}}\right)^{p},\left[K, L_{n}^{\mathfrak{p}}\right],\left[K, \gamma_{n}^{\mathfrak{p}}(Q)\right],\left[L_{n}^{\mathfrak{p}}, Q\right]\right\rangle .
$$

Clearly, the series $L^{\mathfrak{p}}=\left\{L_{n}^{\mathfrak{p}}\right\}_{n \geqslant 1}$ is a p-torsion series, in the sense that $\left(L_{n}^{\mathfrak{p}}\right)^{p} \subseteq L_{n+1}^{\mathfrak{p}}$ for all $n \geqslant 1$. Moreover, we show in [66] that this is an $\mathrm{N}$-series for $K$.

Theorem 7.7 ([66]). Let $G=K \rtimes_{\varphi} Q$ be a split extension of groups and let $p$ be a prime. For each $n \geqslant 1$, there is then a split extension, $\gamma_{n}^{p}(G)=L_{n}^{p} \rtimes_{\varphi} \gamma_{n}^{p}(Q)$.

When $Q$ acts trivially on $H_{1}\left(K ; \mathbb{Z}_{p}\right)$, we show in [66] that $L^{p}=\gamma^{p}(K)$. The next result (originally proved in [5]) then follows from Theorem 7.7.

Theorem 7.8 ([5]). Let $G=K \rtimes_{\varphi} Q$ be a split extension, and assume $Q$ acts trivially on $H_{1}\left(K ; \mathbb{Z}_{p}\right)$. Then,

(1) $\gamma_{n}^{p}(G)=\gamma_{n}^{p}(K) \rtimes_{\varphi} \gamma_{n}^{p}(Q)$, for all $n \geqslant 1$.

(2) $\operatorname{gr}^{p}(G)=\operatorname{gr}^{p}(K) \rtimes_{\bar{\varphi}} \operatorname{gr}^{p}(Q)$.

Under additional assumptions, we have the following corollary, which will be needed in the proof of Theorem 10.4.

Corollary 7.9. Let $G=K \rtimes_{\varphi} Q$ be a split extension of groups. Assume $Q$ is an elementary abelian p-group which acts trivially on $H_{1}\left(K ; \mathbb{Z}_{p}\right)$. Then,

(1) $\gamma_{n}^{p}(K)=\gamma_{n}^{p}(G)$ for all $n \geqslant 2$ and $\operatorname{gr}_{\geqslant 2}^{p}(K)=\operatorname{gr}_{\geqslant 2}^{p}(G)$.

(2) If, moreover, $b_{1}^{p}(G)<\infty$, then $\phi_{n}^{p}(K)=\phi_{n}^{p}(G)$ for all $n \geqslant 2$.

Proof. Since $Q$ is an elementary abelian $p$-group, Example 5.3 shows that $\gamma_{n}^{p}(Q)=\{1\}$ for all $n \geqslant 2$. The first claim now follows from Theorem 7.8.

Next, assume that $b_{1}^{p}(G)<\infty$. Then, by the discussion from $\S 5.3$, all the graded pieces of $\operatorname{gr}^{p}(G)$ are finite-dimensional $\mathbb{Z}_{p}$-vector spaces. Recalling that $\phi_{n}^{p}(G)=\operatorname{dim}_{\mathbb{Z}_{p}} \operatorname{gr}_{n}^{p}(G)$, the second claim now follows from claim (1). 


\section{8. Ав-EXAct SEQUences}

We now return to the general setting of not necessarily split extensions, and extend the notion of almost direct product to this broader context. For the resulting extensions, we prove one of our main results, which relates the Alexander invariant of a normal subgroup $K \triangleleft G$ to that of $G$, under suitable assumptions on the quotient group, $Q=G / K$.

8.1. Ab-exact sequences. A useful tool in the homological study of group extensions is the 5-term exact sequence of Stallings [59] (see also [33, Ch. VI]). Given an extension such as (38), there is an associated exact sequence,

$$
H_{2}(G ; \mathbb{Z}) \stackrel{\pi_{*}}{\longrightarrow} H_{2}(Q ; \mathbb{Z}) \stackrel{\delta}{\longrightarrow} H_{1}(K ; \mathbb{Z})_{Q} \stackrel{\iota *}{\longrightarrow} H_{1}(G ; \mathbb{Z}) \stackrel{\pi_{*}}{\longrightarrow} H_{1}(Q ; \mathbb{Z}) \longrightarrow 0,
$$

where $H_{1}(K ; \mathbb{Z})_{Q}=K /[G, K]$ denotes the coinvariants under the action of $Q$ on $K_{\mathrm{ab}}$ described in $\S 6.1$. When this action is trivial, the group in the middle coincides with $H_{1}(K ; \mathbb{Z})$.

Lemma 8.1. For a group extension, $1 \longrightarrow K \stackrel{\iota}{\longrightarrow} G \stackrel{\pi}{\longrightarrow} Q \longrightarrow 1$, the following two conditions are equivalent.

(1) The group $Q$ acts trivially on $K_{\mathrm{ab}}$ and the map $\delta: H_{2}(Q ; \mathbb{Z}) \rightarrow H_{1}(K ; \mathbb{Z})$ is zero.

(2) The sequence $0 \longrightarrow K_{\mathrm{ab}} \stackrel{\iota_{\mathrm{ab}}}{\longrightarrow} G_{\mathrm{ab}} \stackrel{\pi_{\mathrm{ab}}}{\longrightarrow} Q_{\mathrm{ab}} \longrightarrow 0$ is exact.

Proof. The implication (1) $\Rightarrow$ (2) follows at once from Stallings' exact sequence (43).

For the reverse implication, recall that the monodromy of the extension, $\varphi: Q \rightarrow$ $\operatorname{Aut}\left(K_{\mathrm{ab}}\right)$, is given by $\varphi(x)(a)=\sigma(x) a \sigma(x)^{-1}$, for some choice of set-theoretic section $\sigma: Q \rightarrow G$. Conjugation by any element of $G$ acts trivially on $G_{\mathrm{ab}}$. On the other hand, our assumption guarantees that $K_{\mathrm{ab}}$ injects into $G_{\mathrm{ab}}$. Hence, conjugation by an element of $G$ also acts trivially on $K_{\mathrm{ab}}$. The vanishing of the connecting homomorphism $\delta$ now follows from the injectivity of $\iota_{\mathrm{ab}}=\iota_{*}$.

Following [19], we say that a sequence $1 \rightarrow K \rightarrow G \rightarrow Q \rightarrow 1$ is ab-exact if any one of the two equivalent conditions of Lemma 8.1 is satisfied.

Remark 8.2. Suppose the extension is central, i.e, $K$ lies in the center of $G$. Then $Q$ acts trivially on $K=K_{\mathrm{ab}}$, and the connecting homomorphism $\delta: H_{2}(Q ; \mathbb{Z}) \rightarrow K$ corresponds via the universal coefficients theorem to the cohomology class $\bar{\delta} \in H^{2}(Q ; K)$ that classifies the central extension. Consequently, if a central extension is non-trivial, then the map $\delta$ is not zero, and so the extension is not ab-exact. For instance, if $G$ is the Heisenberg group from Example 5.4, then $G=F_{2} / \gamma_{3} F_{2}$, and so $G$ is a central extension of $G_{\mathrm{ab}} \cong \mathbb{Z}^{2}$ by $G^{\prime} \cong \mathbb{Z}$, with extension class $\bar{\delta}$ a generator of $H^{2}\left(\mathbb{Z}^{2} ; \mathbb{Z}\right)=\mathbb{Z}$.

Next, we give an example of a non-central, non-split, ab-exact extension. We thank Thomas Koberda for help with finding this example. 
Example 8.3. Consider the 2-step nilpotent group $G=\langle a, b, x, y, z|[x, y]=[a, b]=$ $z,[x, a]=[x, b]=[y, a]=[y, b]=[x, z]=[y, z]=[a, z]=[b, z]=1\rangle$. We then have a short exact sequence, $1 \rightarrow K \rightarrow G \rightarrow \mathbb{Z}^{2} \rightarrow 1$, where $K=\langle x, y, z|[x, y]=$ $z,[x, z]=[y, z]=1\rangle$ is the Heisenberg group. It is readily seen that this is a central, nonsplit extension. Moreover, $H_{2}\left(\mathbb{Z}^{2}\right)=\bigwedge^{2} \mathbb{Z}^{2}=\mathbb{Z}$, generated by $a \wedge b$, and $H_{1}(K)=\mathbb{Z}^{2}$, generated by $x, y$; thus, the map $\delta$ is equal to zero.

For split exact sequences, we have the following criterion for determining ab-exactness.

Proposition 8.4. A split exact sequence $1 \rightarrow K \rightarrow G \rightarrow Q \rightarrow 1$ is ab-exact if and only if $Q$ acts trivially on $K_{\mathrm{ab}}$; that is, $G=K \rtimes Q$ is an almost direct product.

Proof. The forward implication follows at once from Lemma 8.1, part (1). For the backwards implication, Theorem 7.2, part (2) yields a (split) exact sequence of abelian groups, $0 \rightarrow \mathrm{gr}_{1}(K) \rightarrow \mathrm{gr}_{1}(G) \rightarrow \mathrm{gr}_{1}(Q) \rightarrow 0$, and this sequence clearly coincides with the one from Lemma 8.1, part (2).

It is easy to build split extensions which are not ab-exact; the fundamental group of the Klein bottle, $G=\left\langle t, a \mid t a t^{-1}=a^{-1}\right\rangle$, is of this sort. Here is a construction that produces a large class of extensions which are both split exact and ab-exact.

Example 8.5. Let $G_{\Gamma}=\langle v \in V|[v, w]=1$ if $\left.\{v, w\} \in E\right\rangle$ be the right-angled Artin group associated to a finite (simple) graph $\Gamma$ on vertex set $V$ and edge set $E$. To avoid trivialities, we will always assume that $|V|>1$. Consider the homomorphism $\pi: G_{\Gamma} \rightarrow \mathbb{Z}$ that sends each generator $v \in V$ to $1 \in \mathbb{Z}$, and let $N_{\Gamma}=\operatorname{ker}(\pi)$ be the corresponding Bestvina-Brady group. We then have a split exact sequence,

$$
1 \longrightarrow N_{\Gamma} \stackrel{\iota}{\longrightarrow} G_{\Gamma} \stackrel{\pi}{\longrightarrow} \mathbb{Z} \longrightarrow 1 \text {. }
$$

As shown in [7], the group $N_{\Gamma}$ is finitely generated if and only if $\Gamma$ is connected; likewise, $N_{\Gamma}$ is finitely presented if and only if the flag complex $\Delta_{\Gamma}$ is simply connected. Furthermore, as shown in [47, Proposition 5.3], if $\Gamma$ is connected, then the group $\mathbb{Z}$ acts trivially on $H_{1}\left(N_{\Gamma} ; \mathbb{Z}\right)$, and so this sequence is ab-exact.

8.2. Ab-exact sequences and Alexander invariants. We now relate the Alexander invariant and the derived subalgebra of the associated graded Lie algebra of a group $G$ with those of a normal subgroup $K \triangleleft G$, provided the quotient group $Q=G / K$ is abelian, and the resulting extension is ab-exact. We start with a lemma.

Lemma 8.6. If $1 \rightarrow K \stackrel{\iota}{\rightarrow} G \stackrel{\pi}{\rightarrow} Q \rightarrow 1$ is an ab-exact sequence, then its restriction to derived subgroups, $1 \rightarrow K^{\prime} \stackrel{\iota^{\prime}}{\rightarrow} G^{\prime} \stackrel{\pi^{\prime}}{\rightarrow} Q^{\prime} \rightarrow 1$, is an exact sequence.

Proof. Follows from the exactness of the sequence $0 \longrightarrow K_{\mathrm{ab}} \stackrel{\iota_{\mathrm{ab}}}{\longrightarrow} G_{\mathrm{ab}} \stackrel{\pi_{\mathrm{ab}}}{\longrightarrow} Q_{\mathrm{ab}} \longrightarrow 0$ and the Nine Lemma in the category of groups. 
For a homomorphism $\psi: G \rightarrow H$, we let $\bar{\psi}: G / G^{\prime \prime} \rightarrow H / H^{\prime \prime}$ be the induced homomorphism on maximal metabelian quotients.

Theorem 8.7. Let $1 \rightarrow K \stackrel{\iota}{\longrightarrow} G \stackrel{\pi}{\rightarrow} Q \rightarrow 1$ be an ab-exact sequence. Assuming $Q$ is abelian, the following hold.

(1) The inclusion $\iota: K \hookrightarrow G$ restricts to an equality, $K^{\prime}=G^{\prime}$.

(2) The induced map on Alexander invariants, $B(\iota): B(K) \rightarrow B(G)$, gives rise to a $\mathbb{Z}\left[K_{\mathrm{ab}}\right]$-linear isomorphism, $B(K) \rightarrow B(G)_{\iota}$.

(3) The sequence $1 \longrightarrow K / K^{\prime \prime} \stackrel{\bar{\iota}}{\longrightarrow} G / G^{\prime \prime} \stackrel{\bar{\pi}}{\longrightarrow} Q \rightarrow 1$ is also ab-exact.

(4) If, moreover, $G_{\mathrm{ab}}$ is finitely generated, then $\theta_{n}(K) \leqslant \theta_{n}(G)$ for all $n \geqslant 1$.

Proof. (1) By Lemma 8.6, we have an exact sequence, $1 \rightarrow K^{\prime} \stackrel{\iota^{\prime}}{\rightarrow} G^{\prime} \stackrel{\pi^{\prime}}{\rightarrow} Q^{\prime} \rightarrow 1$. But $Q^{\prime}=\{1\}$ by assumption, and so $K^{\prime}=G^{\prime}$.

(2) Since $K^{\prime}=G^{\prime}$, we must also have $\left(K^{\prime}\right)^{\prime}=\left(G^{\prime}\right)^{\prime}$. Hence, $K^{\prime} / K^{\prime \prime}=G^{\prime} / G^{\prime \prime}$, showing that the map $B(K) \rightarrow B(G)_{\iota}$ is indeed a $\mathbb{Z}\left[K_{\mathrm{ab}}\right]$-linear isomorphism.

(3) As we just saw, $K^{\prime \prime}=G^{\prime \prime}$; hence, the map $\bar{\imath}$ is injective, and so the sequence in question is exact. Under the identifications $\left(K / K^{\prime \prime}\right)_{\mathrm{ab}}=K_{\mathrm{ab}},\left(G / G^{\prime \prime}\right)_{\mathrm{ab}}=G_{\mathrm{ab}}$, and $Q_{\mathrm{ab}}=Q$, the maps $\bar{\iota}_{\mathrm{ab}}$ and $\bar{\pi}_{\mathrm{ab}}$ coincide with $\iota_{\mathrm{ab}}$ and $\pi_{\mathrm{ab}}$, respectively, and the claim follows.

(4) By our ab-exactness assumption, the homomorphism $\iota_{\mathrm{ab}}: K_{\mathrm{ab}} \rightarrow G_{\mathrm{ab}}$ is injective; therefore, $\theta_{1}(K) \leqslant \theta_{1}(G)$ and $\tilde{\iota}_{\mathrm{ab}}$ is also injective. It follows from part (2) that the map $B(\iota): B(K) \rightarrow B(G)$ factors as an isomorphism of $\mathbb{Z}\left[K_{\mathrm{ab}}\right]$-modules, $B(K) \stackrel{\simeq}{\longrightarrow} B(G)_{\iota}$, followed by the identity map of $B(G)$, viewed as covering the ring map $\tilde{\iota}_{\mathrm{ab}}$.

Passing now to associated graded modules, the morphism $\operatorname{gr}(B(\iota)): \operatorname{gr}(B(K)) \rightarrow$ $\operatorname{gr}(B(G))$ factors as an isomorphism of $\operatorname{gr}\left(\mathbb{Z}\left[K_{\mathrm{ab}}\right]\right)$-modules, followed by the identity map of $\operatorname{gr}(B(G))$, viewed as covering the ring map $\operatorname{gr}\left(\tilde{\iota}_{\mathrm{ab}}\right)$. By Lemma 6.4, $\operatorname{gr}\left(\tilde{\iota}_{\mathrm{ab}}\right)$ is injective; therefore, $\operatorname{gr}(B(\iota))$ is also injective, and so the rank of $\operatorname{gr}_{n}(B(K))$ is less or equal to the rank of $\operatorname{gr}_{n}(B(G))$ for $n \geqslant 0$. It now follows from formula (39) that $\theta_{n}(K) \leqslant \theta_{n}(G)$ for $n \geqslant 2$, and we are done.

When the above ab-exact sequence is also split exact, more can be said.

Corollary 8.8. Let $1 \rightarrow K \stackrel{\iota}{\rightarrow} G \stackrel{\pi}{\rightarrow} Q \rightarrow 1$ be a split exact and ab-exact sequence, and assume $Q$ is abelian. Then

(1) The map เ induces isomorphisms of graded Lie algebras, $\operatorname{gr}_{\geqslant 2}(K) \stackrel{\simeq}{\longrightarrow} \mathrm{gr}_{\geqslant 2}(G)$ and $\operatorname{gr}_{\geqslant 2}\left(K / K^{\prime \prime}\right) \stackrel{\simeq}{\longrightarrow} \operatorname{gr}_{\geqslant 2}\left(G / G^{\prime \prime}\right)$.

(2) If, moreover, $b_{1}(G)<\infty$, then $\phi_{n}(K)=\phi_{n}(G)$ and $\theta_{n}(K)=\theta_{n}(G)$ for all $n \geqslant 2$.

Proof. Let $\sigma: Q \rightarrow G$ be a splitting of $\pi$. By Theorem 8.7, part (3), the extension $1 \rightarrow$ $K / K^{\prime \prime} \rightarrow G / G^{\prime \prime} \rightarrow Q \rightarrow 1$ is ab-exact; it is also split exact, with splitting obtained by composing the projection $G \rightarrow G / G^{\prime \prime}$ with $\sigma$. Thus, by Proposition 8.4 , both extensions 
are almost direct products. Since $Q$ is assumed to be abelian, both claims now follow from Corollary 7.3.

Remark 8.9. For extensions of the form $1 \rightarrow N_{\Gamma} \rightarrow G_{\Gamma} \rightarrow \mathbb{Z} \rightarrow 1$, with $G_{\Gamma}$ the rightangled Artin group and $N_{\Gamma}$ the Bestvina-Brady group associated to a finite connected graph $\Gamma$ as in Example 8.5, Theorem 8.7, parts (1)-(3) and Corollary 8.8 recover Propositions 4.2 and 5.4 and Theorem 5.6 from [47].

\section{AbF-EXAct SEQuences}

In this section we give analogues of the above results for the rational lower central series, the rational derived series, and the rational Alexander invariant.

9.1. Abf-exact sequences. We start with a lemma/definition, the proof of which is exactly similar to that of Lemma 8.1.

Lemma 9.1. For an exact sequence $1 \rightarrow K \stackrel{\iota}{\rightarrow} G \stackrel{\pi}{\rightarrow} Q \rightarrow 1$, the following two conditions are equivalent.

(1) The group $Q$ acts trivially on $K_{\mathrm{abf}}$ and the composite $\delta_{0}: H_{2}(Q ; \mathbb{Z}) \stackrel{\delta}{\rightarrow} H_{1}(K ; \mathbb{Z}) \rightarrow$ $H_{1}(K ; \mathbb{Z}) /$ Tors is zero.

(2) The sequence $0 \longrightarrow K_{\mathrm{abf}} \stackrel{\mathrm{labf}^{\mathrm{ab}}}{\longrightarrow} G_{\mathrm{abf}} \stackrel{\pi_{\mathrm{abf}}}{\longrightarrow} Q_{\mathrm{abf}} \longrightarrow 0$ is exact.

We say that the above sequence is abf-exact if any one of those two conditions is satisfied. Evidently, ab-exactness implies abf-exactness, though the converse is not true, as illustrated by the infinite dihedral group, $D_{\infty}=\mathbb{Z}_{2} \rtimes \mathbb{Z}$.

Let $\delta_{\mathbb{Q}}: H_{2}(Q ; \mathbb{Q}) \rightarrow H_{1}(K ; \mathbb{Q})$ be the connecting homomorphism in the 5-term exact sequence (43) with $\mathbb{Q}$-coefficients. In the case when $K_{\mathrm{ab}}$ has finite rank, we have the following, more convenient criterion for abf-exactness.

Lemma 9.2. Let $1 \rightarrow K \rightarrow G \rightarrow Q \rightarrow 1$ be a group extension, and suppose $K_{\mathrm{abf}}$ is finitely generated. Then the extension is abf-exact if and only if $Q$ acts trivially on $H_{1}(K ; \mathbb{Q})$ and the map $\delta_{\mathbb{Q}}: H_{2}(Q ; \mathbb{Q}) \rightarrow H_{1}(K ; \mathbb{Q})$ is zero.

Proof. First note that $H_{1}(K ; \mathbb{Q})=K_{\text {abf }} \otimes \mathbb{Q}$ and the action of $Q$ on $H_{1}(K ; \mathbb{Q})$ is obtained by extension of scalars from the action of $Q$ on $K_{\mathrm{abf}}$. Likewise, the map $\delta_{\mathbb{Q}}$ is obtained by extension of scalars from $\delta_{0}$. The forward implication follows at once (for any $K$ ).

For the reverse implication, note that our assumption on $K_{\text {abf }}$ implies that this group is a (maximal rank) lattice in the finite-dimensional $\mathbb{Q}$-vector space $H_{1}(K ; \mathbb{Q})=K_{\text {abf }} \otimes \mathbb{Q}$. Thus, if $Q$ acts trivially on $H_{1}(K ; \mathbb{Q})$, it must also act trivially on $K_{\text {abf }}$, and likewise, if $\delta_{\mathbb{Q}}=0$, then $\delta_{0}=0$.

Without the finite generation assumption on $K_{\mathrm{abf}}$, the triviality of the action of $Q$ on $H_{1}(K ; \mathbb{Q})$ does not insure triviality of the action on $K_{\text {abf }}$. We illustrate this phenomenon with an example. 
Example 9.3. For each $n \geqslant 2$, let $G=\mathrm{BS}(1, n)$ be the metabelian Baumslag-Solitar group with presentation $G=\left\langle t, a \mid t a t^{-1}=a^{n}\right\rangle$. In the extension $1 \rightarrow G^{\prime} \rightarrow G \rightarrow G_{\mathrm{ab}} \rightarrow$ 1 , the abelianization is isomorphic to $\mathbb{Z}$, generated by the image of $t$, while the derived subgroup is isomorphic to $\mathbb{Z}[1 / n]$, normally generated by $a$. Thus, the extension is split exact, with monodromy given by $a \mapsto a^{n}$. Clearly, $\mathbb{Z}$ acts trivially on $\mathbb{Z}[1 / n] \otimes \mathbb{Q}=\mathbb{Q}$, though it acts non-trivially on the torsion-free, yet non-finitely generated abelian group $\left(G^{\prime}\right)_{\mathrm{abf}}=\mathbb{Z}[1 / n]$.

Nevertheless, we have the following criteria that insure abf-exactness of a split exact sequence.

Proposition 9.4. Let $1 \rightarrow K \rightarrow G \rightarrow Q \rightarrow 1$ be a split exact sequence.

(1) The sequence is abf-exact if and only if $Q$ acts trivially on $K_{\mathrm{abf}}$.

(2) If $K_{\mathrm{abf}}$ is finitely generated, then the sequence is abf-exact if and only if $Q$ acts trivially on $H_{1}(K ; \mathbb{Q})$.

Proof. The proof of the first claim is similar to the one of Proposition 8.4, using Theorem 7.5, part (2), instead. The second claim now follows from Lemma 9.2.

We call semidirect products $G=K \rtimes_{\varphi} Q$ that satisfy condition (1) from above, rational almost-direct products. Clearly, any split extension $G=K \rtimes Q$ with $K$ finite is of this type. Using Proposition 5.1 and Theorem 7.5, we obtain the following corollary.

Corollary 9.5. Let $G=K \rtimes_{\varphi} Q$ be a split extension such that $K_{\mathrm{abf}}$ is finitely generated and $Q$ acts trivially on $H_{1}(K ; \mathbb{Q})$. Then $\operatorname{gr}(G) \otimes \mathbb{Q}=\operatorname{gr}(K) \otimes \mathbb{Q} \rtimes_{\bar{\varphi}} \operatorname{gr}(Q) \otimes \mathbb{Q}$.

Example 9.6. Let $\Gamma$ be a connected, finite simple graph, and let $\chi: G_{\Gamma} \rightarrow \mathbb{Z}$ be an arbitrary epimorphism. The subgroup $N_{\chi}:=\operatorname{ker}(\chi)$ is called an Artin kernel; it generalizes the Bestvina-Brady construction, and fits into a split exact sequence,

$$
1 \longrightarrow N_{\chi} \stackrel{\iota}{\longrightarrow} G_{\Gamma} \stackrel{\chi}{\longrightarrow} \mathbb{Z} \longrightarrow 1 \text {. }
$$

Suppose $\mathbb{Z}$ acts trivially on $H_{1}\left(N_{\chi} ; \mathbb{Q}\right)$. Then, as shown in [48, Lemma 9.1(1)], the group $N_{\chi}$ is finitely generated; thus, by Proposition 9.4, part (2), the above sequence is abfexact. As shown in [48, Lemma 9.1(3)], the group $\mathbb{Z}$ also acts trivially on $H_{1}\left(N_{\chi} ; \mathbb{Z}\right)$, and so the sequence (45) is actually ab-exact. Applying Corollary 8.8 in this setting recovers Proposition 9.2 from [48].

As we just saw, for extensions of type (45), there is no difference between ab-exactness and abf-exactness. In general, though the two concepts are different, even when the group $K$ is torsion-free. An example is provided by $G=K \rtimes \mathbb{Z}$ with $K=\left\langle t, a \mid t a t^{-1}=a^{-1}\right\rangle$ and $\mathbb{Z}=\langle u\rangle$ acting by $u t u^{-1}=t a$ and $u a u^{-1}=a$, which is a rational almost direct product, but not an almost direct product. 
9.2. Abf-exact sequences and Alexander invariants. We now relate the rational Alexander invariant and the derived rational associated graded Lie algebra of a group $G$ to those of a normal subgroup $K \triangleleft G$, provided the quotient group $Q=G / K$ is torsion-free abelian and the resulting extension is abf-exact. We start with a lemma, whose proof is similar to that of Lemma 8.6.

Lemma 9.7. If $1 \rightarrow K \stackrel{\iota}{\rightarrow} G \stackrel{\pi}{\rightarrow} Q \rightarrow 1$ is an abf-exact sequence, then its restriction to $\mathbb{Q}$-derived subgroups, $1 \rightarrow K_{\mathbb{Q}}^{\prime} \stackrel{\iota^{\prime}}{\rightarrow} G_{Q}^{\prime} \stackrel{\pi^{\prime}}{\rightarrow} Q_{Q}^{\prime} \rightarrow 1$, is an exact sequence.

Theorem 9.8. Let $1 \rightarrow K \stackrel{\iota}{\rightarrow} G \stackrel{\pi}{\rightarrow} Q \rightarrow 1$ be an abf-exact sequence, and assume $Q$ is a torsion-free abelian group. Then,

(1) The inclusion $\iota: K \hookrightarrow G$ restricts to an equality $K_{\mathrm{Q}}^{\prime}=G_{\mathrm{Q}}^{\prime}$.

(2) The induced map on rational Alexander invariants, $B_{Q}(\iota): B_{Q}(K) \rightarrow B_{Q}(G)$, gives rise to a $\mathbb{Z}\left[K_{\mathrm{abf}}\right]$-linear isomorphism, $B_{\mathrm{Q}}(K) \rightarrow B_{\mathrm{Q}}(G)_{\iota}$.

(3) The sequence $1 \rightarrow K / K_{\mathbb{Q}}^{\prime \prime} \stackrel{\bar{\iota}}{\rightarrow} G / G_{\mathbb{Q}}^{\prime \prime} \stackrel{\bar{\pi}}{\rightarrow} Q \rightarrow 0$ is also abf-exact.

(4) If, moreover, $G_{\text {abf }}$ is finitely generated, then $\theta_{n}(K) \leqslant \theta_{n}(G)$ for all $n \geqslant 1$.

Proof. (1) By Lemma 9.7, we have an exact sequence, $1 \rightarrow K_{Q}^{\prime} \stackrel{\iota^{\prime}}{\rightarrow} G_{Q}^{\prime} \stackrel{\pi^{\prime}}{\rightarrow} Q_{Q}^{\prime} \rightarrow 1$. But $Q_{\mathrm{Q}}^{\prime}=\{1\}$ by assumption, and so $K_{\mathrm{Q}}^{\prime}=G_{\mathrm{Q}}^{\prime}$.

(2) It follows that $K_{\mathrm{Q}}^{\prime \prime}=G_{\mathrm{Q}}^{\prime \prime}$, too, and so $K_{\mathrm{Q}}^{\prime} / K_{\mathrm{Q}}^{\prime \prime}=G_{\mathrm{Q}}^{\prime} / G_{\mathrm{Q}}^{\prime \prime}$, whence the claim.

(3) As we just saw, $K_{0}^{\prime \prime}=G_{0}^{\prime \prime}$; hence, the map $\bar{\imath}$ is injective, and so the sequence in question is exact. Under the identifications $\left(K / K_{\mathrm{Q}}^{\prime \prime}\right)_{\mathrm{abf}}=K_{\mathrm{abf}}$ and $\left(G / G_{\mathrm{Q}}^{\prime \prime}\right)_{\mathrm{abf}}=G_{\mathrm{abf}}$, the map $\bar{\iota}_{\text {abf }}$ coincides with $\iota_{\text {abf }}$, and the claim follows.

(4) By assumption, the map $\iota_{\mathrm{abf}}: K_{\mathrm{abf}} \rightarrow G_{\mathrm{abf}}$ is injective; therefore, its extension to group rings, $\tilde{\iota}_{\mathrm{abf}}: \mathbb{Z}\left[K_{\mathrm{abf}}\right] \rightarrow \mathbb{Z}\left[G_{\mathrm{abf}}\right]$, is also injective, and $\theta_{1}(K) \leqslant \theta_{1}(G)$. It follows from part (2) that the map $B_{\mathbb{Q}}(\iota): B_{\mathbb{Q}}(K) \rightarrow B_{\mathbb{Q}}(G)$ factors as an isomorphism of $\mathbb{Z}\left[K_{\mathrm{abf}}\right]$ modules, $B_{\mathrm{Q}}(K) \stackrel{\simeq}{\longrightarrow} B_{\mathrm{Q}}(G)_{\iota}$, followed by the identity map of $B_{\mathrm{Q}}(G)$, viewed as covering the ring map $\tilde{\iota}_{\text {abf }}$. Hence, the morphism $\operatorname{gr}\left(B_{Q}(\iota)\right): \operatorname{gr}\left(B_{Q}(K)\right) \rightarrow \operatorname{gr}\left(B_{Q}(G)\right)$ factors as an isomorphism of $\operatorname{gr}\left(\mathbb{Z}\left[K_{\mathrm{abf}}\right]\right)$-modules, followed by the identity map of $\operatorname{gr}\left(B_{\mathrm{Q}}(G)\right)$, viewed as covering the ring map $\operatorname{gr}\left(\tilde{\iota}_{\mathrm{abf}}\right)$. The proof of Lemma 6.4 shows that $\operatorname{gr}\left(\tilde{\iota}_{\mathrm{abf}}\right)$ is injective; therefore, $\operatorname{gr}\left(B_{Q}(\iota)\right)$ is also injective. It now follows from Corollary 6.8 that $\theta_{n}(K) \leqslant \theta_{n}(G)$ for $n \geqslant 2$, and we are done.

When the above abf-exact sequence is also split exact, more can be said.

Corollary 9.9. Let $1 \rightarrow K \stackrel{\iota}{\rightarrow} G \stackrel{\pi}{\rightarrow} Q \rightarrow 1$ be a split exact and abf-exact sequence, and assume $Q$ is torsion-free abelian. Then

(1) The map เ induces isomorphisms of graded Lie algebras, $\operatorname{gr}_{\geqslant 2}^{\circ}(K) \stackrel{\simeq}{\longrightarrow} \operatorname{gr}_{\geqslant 2}^{\circ}(G)$ and $\mathrm{gr}_{\geqslant 2}^{\mathrm{e}}\left(K / K^{\prime \prime}\right) \stackrel{\simeq}{\longrightarrow} \mathrm{gr}_{\geqslant 2}^{\mathrm{e}}\left(G / G^{\prime \prime}\right)$.

(2) If $b_{1}(G)<\infty$, then $\phi_{n}(K)=\phi_{n}(G)$ and $\theta_{n}(K)=\theta_{n}(G)$ for all $n \geqslant 2$. 
Proof. By assumption, the extension $1 \rightarrow K \rightarrow G \rightarrow Q \rightarrow 1$ is abf-exact and admits a splitting, say, $\sigma: Q \rightarrow G$. By Theorem 9.8, part (3), the extension $1 \rightarrow K / K_{\mathrm{Q}}^{\prime \prime} \rightarrow$ $G / G_{0}^{\prime \prime} \rightarrow Q \rightarrow 1$ is abf-exact; it is also split exact, with splitting obtained by composing the projection $G \rightarrow G / G_{\mathbb{Q}}^{\prime \prime}$ with $\sigma$. Thus, by Proposition 9.4 , both extensions are $\mathbb{Q}$ almost direct products. Since the group $Q$ is assumed to be torsion-free abelian, both claims now follow from Corollary 7.6.

The above corollary has the following topological consequence.

Corollary 9.10. Let $X$ be a connected $C W$-complex such that $b_{1}(X)<\infty$, let $f: X \rightarrow X$ be a map inducing the identity on $H_{1}(X ; \mathbb{Q})$, and let $T_{f}$ be the mapping torus of $f$. Then $\phi_{n}\left(\pi_{1}(X)\right)=\phi_{n}\left(\pi_{1}\left(T_{f}\right)\right)$ and $\theta_{n}\left(\pi_{1}(X)\right)=\theta_{n}\left(\pi_{1}\left(T_{f}\right)\right)$ for all $n \geqslant 2$.

\section{0. $p$-EXAct SEQUENCES}

In this section we give analogues of the results from the previous two sections for the mod- $p$ lower central series, the derived $p$-series, and the mod- $p$ Alexander invariant. We start with a lemma/definition. Let $\delta_{p}: H_{2}\left(Q ; \mathbb{Z}_{p}\right) \rightarrow H_{1}\left(K ; \mathbb{Z}_{p}\right)$ be the connecting homomorphism in the 5-term Stallings exact sequence (43) with $\mathbb{Z}_{p}$-coefficients.

Lemma 10.1. For an exact sequence $1 \rightarrow K \stackrel{\iota}{\rightarrow} G \stackrel{\pi}{\rightarrow} Q \rightarrow 1$ and a prime $p$, the following two conditions are equivalent.

(1) The group $Q$ acts trivially on $H_{1}\left(K ; \mathbb{Z}_{p}\right)$ and the homomorphism $\delta_{p}: H_{2}\left(Q ; \mathbb{Z}_{p}\right) \rightarrow$ $H_{1}\left(K ; \mathbb{Z}_{p}\right)$ is zero.

(2) The sequence $0 \longrightarrow H_{1}\left(K ; \mathbb{Z}_{p}\right) \stackrel{\iota_{*}}{\longrightarrow} H_{1}\left(G ; \mathbb{Z}_{p}\right) \stackrel{\pi_{*}}{\longrightarrow} H_{1}\left(Q ; \mathbb{Z}_{p}\right) \longrightarrow 0$ is exact.

We say that the sequence $1 \rightarrow K \rightarrow G \rightarrow Q \rightarrow 1$ is $p$-exact if any one of the above two conditions is satisfied. A non-example is given by the central, non-split extension $0 \rightarrow \mathbb{Z}_{p} \rightarrow \mathbb{Z}_{p^{2}} \rightarrow \mathbb{Z}_{p} \rightarrow 0$; the split exact sequence $1 \rightarrow \mathbb{Z}_{3} \rightarrow S_{3} \rightarrow \mathbb{Z}_{2} \rightarrow 1$ is 2 -exact but not 3-exact.

Lemma 10.2. If $1 \rightarrow K \rightarrow G \rightarrow Q \rightarrow 1$ is a p-exact sequence, then its restriction to p-derived subgroups, $1 \rightarrow K_{p}^{\prime} \rightarrow G_{p}^{\prime} \rightarrow Q_{p}^{\prime} \rightarrow 1$, is an exact sequence.

Proof. The proof is entirely similar to that of Lemma 8.6.

Proposition 10.3. A split exact sequence $1 \rightarrow K \rightarrow G \rightarrow Q \rightarrow 1$ is p-exact if and only if $Q$ acts trivially on $K_{\mathrm{ab}} \otimes \mathbb{Z}_{p}=H_{1}\left(K ; \mathbb{Z}_{p}\right)$.

Proof. The proof is similar to the proof of Proposition 8.4, using now Theorem 7.8, part (2), instead.

We call semidirect products $G=K \rtimes Q$ such as these mod-p almost-direct products. Clearly, every almost direct product is a mod- $p$ almost-direct product (for any prime $p$ ). But the converse is not true. For instance, take again the Klein bottle group, $G=$ $\left\langle t, a \mid t a t^{-1}=a^{-1}\right\rangle$; then $\mathbb{Z}=\langle t\rangle$ acts non-trivially on $\mathbb{Z}=\langle a\rangle$, but acts trivially on $\mathbb{Z} \otimes \mathbb{Z}_{2}=\mathbb{Z}_{2}$. 
Theorem 10.4. Let $1 \rightarrow K \rightarrow G \rightarrow Q \rightarrow 1$ be a p-exact sequence. Assume $Q$ is an elementary abelian p-group. Then,

(1) The inclusion $\iota: K \hookrightarrow G$ restricts to an equality $K_{p}^{\prime}=G_{p}^{\prime}$.

(2) The induced map on p-Alexander invariants, $B_{p}(K) \rightarrow B_{p}(G)$, gives rise to a $\mathbb{Z}_{p}\left[H_{1}\left(K ; \mathbb{Z}_{p}\right)\right]$-linear isomorphism, $B_{p}(K) \rightarrow B_{p}(G)_{\iota}$.

(3) The sequence $1 \longrightarrow K / K_{p}^{\prime \prime} \stackrel{\bar{\iota}}{\longrightarrow} G / G_{p}^{\prime \prime} \stackrel{\bar{\pi}}{\longrightarrow} Q \rightarrow 0$ is also p-exact.

(4) If, moreover, $b_{1}^{p}(G)<\infty$, then $\theta_{n}^{p}(K) \leqslant \theta_{n}^{p}(G)$ for all $n \geqslant 1$.

Proof. By Lemma 10.2, the given sequence induces an exact sequence at the level of $p$-derived subgroups, $1 \rightarrow K_{p}^{\prime} \rightarrow G_{p}^{\prime} \rightarrow Q_{p}^{\prime} \rightarrow 1$. Since $Q_{p}^{\prime}=\left\langle Q^{p}, Q^{\prime}\right\rangle$, our assumption on $Q$ implies that $Q_{p}^{\prime}=\{1\}$, and the claim is proved.

(2) It follows from that $K_{p}^{\prime \prime}=G_{p}^{\prime \prime}$, too, and so $K_{p}^{\prime} / K_{p}^{\prime \prime}=G_{p}^{\prime} / G_{p}^{\prime \prime}$.

(3) As we just saw, $K_{p}^{\prime \prime}=G_{p}^{\prime \prime}$; hence, the map $\bar{\imath}$ is injective, and so the sequence in question is exact. Under the identifications $H_{1}\left(K / K^{\prime \prime} ; \mathbb{Z}_{p}\right)=H_{1}\left(K ; \mathbb{Z}_{p}\right), H_{1}\left(G / G^{\prime \prime} ; \mathbb{Z}_{p}\right)=$ $H_{1}\left(G, \mathbb{Z}_{p}\right)$, and $H_{1}\left(Q ; \mathbb{Z}_{p}\right)=Q$, the maps $\bar{\imath}$ and $\bar{\pi}$ coincide with $\iota_{*}$ and $\pi_{*}$, respectively, and the claim follows.

(4) By assumption, the map $\iota_{*}: H_{1}\left(K ; \mathbb{Z}_{p}\right) \rightarrow H_{1}\left(G ; \mathbb{Z}_{p}\right)$ is injective; therefore, the map $\tilde{\iota}_{*}: \mathbb{Z}\left[H_{1}\left(K ; \mathbb{Z}_{p}\right)\right] \rightarrow \mathbb{Z}\left[H_{1}\left(G ; \mathbb{Z}_{p}\right)\right]$ is also injective and $\theta_{1}^{p}(K) \leqslant \theta_{1}^{p}(G)$. It follows from part (2) that the map $B_{p}(\iota): B_{p}(K) \rightarrow B_{p}(G)$ factors as an isomorphism of $\mathbb{Z}\left[H_{1}\left(K ; \mathbb{Z}_{p}\right)\right]$-modules, $B_{p}(K) \stackrel{\simeq}{\longrightarrow} B_{p}(G)$, followed by the identity map of $B_{p}(G)$, viewed as covering the ring map $\tilde{\iota}_{*}$. Proceeding as in the proof of Theorem 8.7, part (4), we infer from formula (41) that $\theta_{n}^{p}(K) \leqslant \theta_{n}^{p}(G)$ for $n \geqslant 2$, and we are done.

Corollary 10.5. Let $1 \rightarrow K \rightarrow G \rightarrow Q \rightarrow 1$ be a split exact and p-exact sequence. Assume $Q$ is an elementary abelian p-group. Then,

(1) The inclusion $\iota: K \hookrightarrow G$ induces isomorphisms of graded $\mathbb{Z}_{p}$-Lie algebras, $\operatorname{gr}_{\geqslant 2}^{p}(K) \stackrel{\simeq}{\longrightarrow} \operatorname{gr}_{\geqslant 2}^{p}(G)$ and $\operatorname{gr}_{\geqslant 2}^{p}\left(K / K^{\prime \prime}\right) \stackrel{\simeq}{\longrightarrow} \operatorname{gr}_{\geqslant 2}^{p}\left(G / G^{\prime \prime}\right)$.

(2) If $b_{1}^{p}(G)<\infty$, then $\phi_{n}^{p}(K)=\phi_{n}^{p}(G)$ and $\theta_{n}^{p}(K)=\theta_{n}^{p}(G)$ for all $n \geqslant 2$.

Proof. By Theorem 10.4, part (3), the extension $1 \rightarrow K / K_{p}^{\prime \prime} \rightarrow G / G_{p}^{\prime \prime} \rightarrow Q \rightarrow 1$ is $p$-exact; it is also split exact, with splitting obtained by composing the projection $G \rightarrow G / G_{p}^{\prime \prime}$ with a splitting $Q \rightarrow G$ of the extension $1 \rightarrow K \rightarrow G \rightarrow Q \rightarrow 1$. Thus, by Proposition 10.3, both extensions are $p$-almost direct products. Since $Q$ is an elementary abelian $p$-group and acts trivially on $H_{1}\left(K ; \mathbb{Z}_{p}\right)$, both claims now follow from Corollary 7.9 . 


\section{Part III. Characteristic varieties}

\section{JUMP LOCI FOR RANK 1 LOCAL SYSTEMS}

We now switch our attention to the cohomology jump loci associated to a finitely generated group. We start with a quick review of some of the basic theory of the characteristic varieties.

11.1. A stratification of the character group. Throughout this section, $G$ will be a finitely generated group. The character group, $\mathbb{T}_{G}=\operatorname{Hom}\left(G, \mathbb{C}^{*}\right)$, is an abelian, complex algebraic group, with identity 1 the trivial representation. The coordinate ring of $\mathbb{T}_{G}$ is the group algebra $\mathbb{C}\left[G_{\mathrm{ab}}\right]$; thus, we may identify $\mathbb{T}_{G}$ with $\operatorname{Spec}_{\mathrm{m}}\left(\mathbb{C}\left[G_{\mathrm{ab}}\right]\right)$, the maximal spectrum of this $\mathbb{C}$-algebra. Since each character $\rho: G \rightarrow \mathbb{C}^{*}$ factors through the abelianization $G_{\mathrm{ab}}$, the map ab: $G \rightarrow G_{\mathrm{ab}}$ induces an isomorphism, ab* : $\mathbb{T}_{G_{\mathrm{ab}}} \stackrel{\simeq}{\longrightarrow} \mathbb{T}_{G}$.

Let $X$ be a connected CW-complex with finite 1-skeleton and with $\pi_{1}(X)=G$. Upon identifying a point $\rho \in \mathbb{T}_{G}$ with a rank one local system $\mathbb{C}_{\rho}$ on $X$, we define for each $k \geqslant 1$ the depth $k$ characteristic variety of $G$ as

$$
\mathscr{V}_{k}(G):=\left\{\rho \in \mathbb{T}_{G} \mid \operatorname{dim}_{\mathbb{C}} H_{1}\left(X, \mathbb{C}_{\rho}\right) \geqslant k\right\} .
$$

Clearly, $1 \in \mathscr{V}_{k}(G)$ if and only if $b_{1}(G) \geqslant k$. Furthermore, we have a descending filtration of the character group,

$$
\mathbb{T}_{G} \supseteq \mathscr{V}_{1}(G) \supseteq \mathscr{V}_{2}(G) \supseteq \cdots \supseteq \mathscr{V}_{k}(G) \supseteq \cdots .
$$

Since a classifying space $K(G, 1)$ may be constructed by attaching to $X$ cells of dimension 3 and higher, it is straightforward to verify that the sets $\mathscr{V}_{k}(G)$ do not depend on the choice of space $X$ as above. Furthermore, since $H_{1}\left(X, \mathbb{C}_{\rho}\right) \cong H^{1}\left(X, \mathbb{C}_{\rho^{-1}}\right)$, we may replace in (46) homology with cohomology and obtain the same sets.

Denoting by $\mathbb{T}_{G}^{0}$ the identity component of $\mathbb{T}_{G}$, we have an isomorphism abf* $: \mathbb{T}_{G_{\text {abf }}} \stackrel{\simeq}{\longrightarrow}$ $\mathbb{T}_{G}^{0}$. It is readily seen that $\mathbb{T}_{G}^{0}$ is a complex affine torus of dimension $r=\operatorname{rank} G_{\mathrm{ab}}$, and that $\mathbb{T}_{G}$ is a disjoint union of such tori, indexed by the finite group $\operatorname{Tors}\left(G_{\mathrm{ab}}\right)$. We define the restricted characteristic varieties to be the traces of the depth- $k$ characteristic varieties on this complex torus,

$$
\mathscr{W}_{k}(G):=\mathscr{V}_{k}(G) \cap \mathbb{T}_{G}^{0}
$$

By construction, we have an inclusion $\mathscr{W}_{k}(G) \subseteq \mathscr{V}_{k}(G)$, which becomes an equality if $G_{\mathrm{ab}}$ is torsion-free. The inclusion may be strict, in general.

Example 11.1. Suppose $b_{1}(G)=0$; then $\mathbb{T}_{G}$ is a finite set, in bijection with $G_{\text {ab }}$, while $\mathbb{T}_{G}^{0}=\{1\}$. Although in this case $1 \notin \mathscr{V}_{1}(G)$, and so $\mathscr{W}_{1}(G)=\varnothing$, the set $\mathscr{V}_{1}(G)$ may be non-empty. For instance, if $G=\mathbb{Z}_{2} * \mathbb{Z}_{2}$, then $\mathbb{T}_{G}=\{( \pm 1, \pm 1)\}$ and $\mathscr{V}_{1}(G)=\{(-1,-1)\}$.

11.2. Alexander matrices and Fitting ideals. In Lemma 2.2.3 and Corollary 2.4.3 from [34], E. Hironaka showed that the characteristic varieties of a finitely presented group $G$ are Zariski closed subsets of the character group $\mathbb{T}_{G}$. For completeness, we give 
a quick proof of this result, in the more general context that we have adopted here (see also [24, Proposition 2.4] for a related argument).

Given a finitely generated module $M$ over a commutative ring $R$, we let $\operatorname{Fitt}_{k}(M)$ denote the Fitting ideal of codimension $k-1$ minors in a presentation matrix for $M$. In the next lemma, $M$ will be the Alexander module $A(G)=\mathbb{Z}\left[G_{\mathrm{ab}}\right] \otimes_{\mathbb{Z}[G]} I(G)$ from (8), viewed as a module over the $\operatorname{ring} R=\mathbb{Z}\left[G_{\mathrm{ab}}\right]$.

Lemma 11.2 ([34]). Let $G$ be a finitely generated group. Then, for all $k \geqslant 1$,

$$
\mathscr{V}_{k}(G)=V\left(\operatorname{Fitt}_{k+1}(A(G) \otimes \mathbb{C})\right),
$$

at least away from $1 \in \mathbb{T}_{G}$, with equality at 1 for $k<b_{1}(G)$.

Proof. Pick a presentation for $G$ with generators $x_{1}, \ldots, x_{m}$; let $X$ be the corresponding presentation 2-complex, and let $\left(C \cdot\left(X^{\mathrm{ab}} ; \mathbb{Z}\right), \partial^{\mathrm{ab}}\right)$ be the $\mathbb{Z}\left[G_{\mathrm{ab}}\right]$-equivariant chain complex of the maximal abelian cover $X^{\mathrm{ab}}$, as displayed in (9). By definition, a character $\rho: G_{\mathrm{ab}} \rightarrow \mathbb{C}^{*}$ belongs to $\mathscr{V}_{k}(G)$ precisely when $\operatorname{rank} \partial_{2}^{\mathrm{ab}}(\rho)+\operatorname{rank} \partial_{1}^{\mathrm{ab}}(\rho) \leqslant m-k$, where the evaluation of $\partial_{i}^{\mathrm{ab}}$ at $\rho$ is obtained by applying the ring morphism $\mathbb{Z}\left[G_{\mathrm{ab}}\right] \rightarrow \mathbb{C}$, $g \mapsto \rho(g)$ to each entry. Hence, $\mathscr{V}_{k}(X)$ is the zero-set of the ideal of minors of size $m-k+1$ of the block-matrix $\partial_{2}^{\mathrm{ab}} \oplus \partial_{1}^{\mathrm{ab}}$.

Now, $\partial_{1}^{\mathrm{ab}}(\rho)=0$ if and only if $\rho=1$. Therefore, if $\rho$ is a non-trivial character, then $\rho \in \mathscr{V}_{k}(G)$ if and only if $\operatorname{rank} \partial_{2}^{\mathrm{ab}}(\rho) \leqslant m-k-1$, or, equivalently, all codimension $k$ minors of $\partial_{2}^{\mathrm{ab}}$ vanish when evaluated at $\rho$. But we know from (11) that $A(G)$ is the cokernel of $\partial_{2}^{\mathrm{ab}}$, and so the claim is proved for $\rho \neq 1$. Finally, the evaluation of the chain complex (9) at $\rho=1$ is simply $C .(X ; \mathbb{Z})$, and the last claim follows.

A similar result holds for the restricted characteristic varieties, with the Alexander module replaced by its rational counterpart, $A_{\mathbb{Q}}(G)=\mathbb{Z}\left[G_{\mathrm{abf}}\right] \otimes_{\mathbb{Z}[G]} I(G)$.

Lemma 11.3. Let $G$ be a finitely generated group. Then, for all $k \geqslant 1$,

$$
\mathscr{W}_{k}(G)=V\left(\operatorname{Fitt}_{k+1}\left(A_{\mathbb{Q}}(G) \otimes \mathbb{C}\right)\right),
$$

at least away from $1 \in \mathbb{T}_{G}^{0}$, with equality at 1 for $k<b_{1}(G)$.

Proof. Recall from $\S 3.3$ that $\partial^{\mathrm{abf}}=\partial^{\mathrm{ab}} \otimes_{\mathbb{Z}\left[G_{\mathrm{ab}}\right]} \mathbb{Z}\left[G_{\mathrm{abf}}\right]$. Therefore, for a character $\rho: G_{\text {abf }} \rightarrow \mathbb{C}^{*}$, we have that $\partial^{\mathrm{abf}}(\rho)=\partial^{\mathrm{ab}}(\rho)$. Hence, $\rho$ belongs to $\mathscr{W}_{k}(G)$ precisely when rank $\partial_{2}^{\operatorname{abf}}(\rho)+\operatorname{rank} \partial_{1}^{\mathrm{abf}}(\rho) \leqslant m-k$.

On the other hand, we know from Lemma 3.4 that $A_{\mathbb{Q}}(G) \otimes \mathbb{Q}=\operatorname{coker}\left(\partial_{2}^{\mathrm{abf}} \otimes \mathbb{Q}\right)$. Proceeding as in the proof Lemma 11.2 yields the desired conclusions.

\section{Alexander varieties}

We now relate the characteristic varieties of a finitely generated group $G$ with the support loci of the exterior powers of the Alexander invariant of $G$. 
12.1. Support loci of Alexander invariants. We start with some basic notions from commutative algebra. Let $R$ be a commutative ring, and let $M$ be an $R$-module. The support of $M$, denoted $\operatorname{supp}(M)$, consists of those maximal ideals $m \in \operatorname{Spec}_{\mathrm{m}}(R)$ for which the localization $M_{\mathrm{m}}$ is non-zero. Supports are additive, in the following sense: If $0 \rightarrow M \rightarrow N \rightarrow P \rightarrow 0$ is an exact sequence of $R$-modules, then $\operatorname{supp}(N)=\operatorname{supp}(M) \cup$ $\operatorname{supp}(P)$. We denote by $\operatorname{ann}_{R}(M)$ the annihilator ideal of $M$. The following lemma is well-known; see e.g. [27].

Lemma 12.1. Suppose $M$ is a finitely generated $R$-module. Then

(1) $\operatorname{supp}(M)=V\left(\operatorname{ann}_{R}(M)\right)$.

(2) $V\left(\operatorname{ann}_{R}\left(\bigwedge^{k} M\right)\right)=V\left(\operatorname{Fitt}_{k}(M)\right)$.

In order to analyze in more depth the characteristic varieties $\mathscr{V}_{k}(G)$, it is useful to consider the complexified Alexander invariant $B(G) \otimes \mathbb{C}$, viewed as a module over the ring $\mathbb{C}\left[G_{\mathrm{ab}}\right]$, and its exterior powers, $\bigwedge^{k} B(G) \otimes \mathbb{C}$. The support loci of these modules,

$$
\mathscr{Y}_{k}(G)=\operatorname{supp}\left(\bigwedge^{k} B(G) \otimes \mathbb{C}\right),
$$

are called the Alexander varieties of $G$. By construction, these sets form a descending filtration by Zariski closed subsets of the character group, $\mathbb{T}_{G}=\operatorname{Spec}_{\mathrm{m}}\left(\mathbb{C}\left[G_{\mathrm{ab}}\right]\right)$.

Likewise, the study of the restricted characteristic varieties $\mathscr{W}_{k}(G)$ is related to the $\mathbb{C}\left[G_{\text {abf }}\right]$-module $B_{\odot}(G) \otimes \mathbb{C}$ and its exterior powers. The support loci of these modules,

$$
\mathscr{Z}_{k}(G)=\operatorname{supp}\left(\bigwedge^{k} B_{\mathbb{Q}}(G) \otimes \mathbb{C}\right),
$$

are subvarieties of the character torus, $\mathbb{T}_{G}^{0}=\operatorname{Spec}_{\mathrm{m}}\left(\mathbb{C}\left[G_{\mathrm{abf}}\right]\right)$. The next lemma will be useful in analyzing these support loci.

Lemma 12.2. Let $M$ and $N$ be modules over commutative rings $R$ and $S$, respectively, and let $\psi: M \rightarrow N$ be a surjective morphism covering a surjective ring map, $\varphi: R \rightarrow S$. Then the induced morphism on maximal spectra, $\varphi^{*}: \operatorname{Spec}_{\mathrm{m}}(S) \hookrightarrow \operatorname{Spec}_{\mathrm{m}}(R)$, restricts to embeddings $\operatorname{supp}\left(\bigwedge^{k} N\right) \hookrightarrow \operatorname{supp}\left(\bigwedge^{k} M\right)$ for all $k \geqslant 1$.

Proof. By (13), the map $\psi$ factors as a composite, $M \rightarrow N_{\varphi} \rightarrow N$. Taking exterior powers of the first map, we obtain epimorphisms $\bigwedge_{R}^{k} M \rightarrow \bigwedge_{R}^{k} N_{\varphi}$. Since the ring map $\varphi: R \rightarrow S$ is surjective, the module $\bigwedge_{R}^{k} N_{\varphi}$ is obtained from $\bigwedge_{S}^{k} N$ by restriction of scalars. Therefore, the map $\varphi$ restricts to a surjection, $\operatorname{ann}_{R}\left(\bigwedge_{R}^{k} M\right) \rightarrow \operatorname{ann}_{S}\left(\bigwedge_{S}^{k} N\right)$, and the claim follows.

By Proposition 3.5, we have an epimorphism $\kappa \otimes \mathbb{C}: B(G) \otimes \mathbb{C} \rightarrow B_{\bigotimes}(G) \otimes \mathbb{C}$ which covers the ring map $\tilde{v}: \mathbb{C}\left[G_{\mathrm{ab}}\right] \rightarrow \mathbb{C}\left[G_{\mathrm{abf}}\right]$. Applying the previous lemma, we obtain the following corollary.

Corollary 12.3. For a finitely generated group $G$, the inclusion $\mathbb{T}_{G}^{0} \hookrightarrow \mathbb{T}_{G}$ restricts to inclusions $\mathscr{Z}_{k}(G) \hookrightarrow \mathscr{Y}_{k}(G)$, for all $k \geqslant 1$. 
If $G_{\mathrm{ab}}$ is torsion-free, the map $\kappa \otimes \mathbb{C}$ is an isomorphism, and so $\mathscr{Z}_{k}(G)=\mathscr{Y}_{k}(G)$. As illustrated by the next example, this equality may not hold when $\operatorname{Tors}\left(G_{\mathrm{ab}}\right) \neq 0$.

Example 12.4. Consider again the group $G=\mathbb{Z}_{2} * \mathbb{Z}_{2}$, with character group $\mathbb{T}_{G}=$ $\{( \pm 1, \pm 1)\}$. Then $B(G)=\mathbb{Z}\left[x_{1}^{ \pm 1}, x_{2}^{ \pm 1}\right] /\left(1+x_{1}, 1+x_{2}\right)$, and so $\mathscr{Y}_{1}(G)=\{(-1,-1)\}$, whereas $B_{Q}(G)=0$, and so $\mathscr{Z}_{1}(G)=\varnothing$.

12.2. Exterior powers in exact sequences. The next lemma is the key algebraic ingredient in our analysis of the higher-depth Alexander varieties. The lemma is well-known in the case when $R=\mathbb{k}$ is a field and the modules are finite-dimensional $\mathbb{k}$-vector spaces. Nevertheless, we could not find a reference in the generality that we need here; thus, we provide a detailed proof.

Lemma 12.5. Let $0 \rightarrow M \stackrel{\alpha}{\rightarrow} N \stackrel{\beta}{\rightarrow} P \rightarrow 0$ be an exact sequence of modules over a commutative ring $R$. For each $k \geqslant 1$, the exterior power $\wedge^{k} N$ admits a decreasing filtration by $R$-submodules,

$$
\bigwedge^{k} N=F_{0}^{k} \supseteq F_{1}^{k} \supseteq \cdots \supseteq F_{k+1}^{k}=0,
$$

such that

$$
F_{i}^{k} / F_{i+1}^{k} \cong \bigwedge^{i} M \otimes \bigwedge^{k-i} P
$$

for $0 \leqslant i \leqslant k$.

Proof. For $k \geqslant 1$ and $0 \leqslant i \leqslant k$, define an $R$-linear map $\alpha_{i}^{k}: \bigwedge^{i} M \otimes \bigwedge^{k-i} N \rightarrow \bigwedge^{k} N$ by $\alpha_{i}^{k}(u \otimes v)=\bigwedge^{i} \alpha(u) \wedge v$, and set $F_{i}^{k}:=\operatorname{im}\left(\alpha_{i}^{k}\right)$. Clearly, this defines a filtration on the module $\wedge^{k} N$ such that (51) holds. To show that (52) does also hold, we use [27, Proposition A.2.2(d)], from which we extract the following statement: for each $j \geqslant 1$, there is an exact sequence

$$
M \otimes \bigwedge^{j-1} N \stackrel{\alpha_{1}^{j-1}}{\longrightarrow} \bigwedge^{j} N \stackrel{\bigwedge^{j} \beta}{\longrightarrow} \bigwedge^{j} P \longrightarrow 0 .
$$

(For $j=1$, this is the original exact sequence.) Now fix $i \geqslant 0$ and set $j=k-i$; tensoring the sequence (53) with $\bigwedge^{i} M$, we obtain the exact sequence at the top of the following diagram.

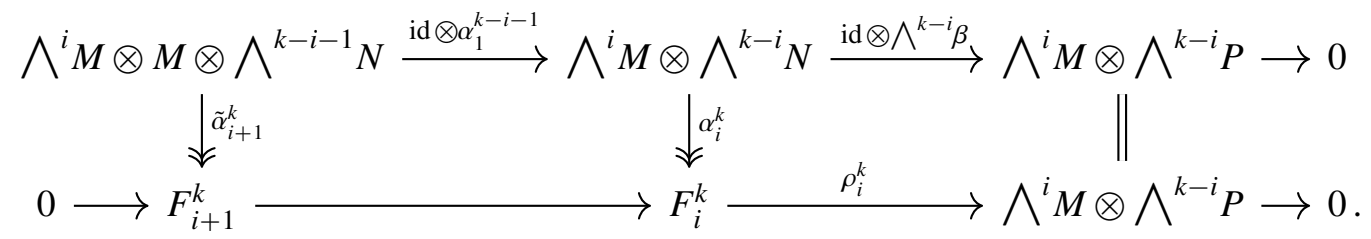

In this diagram, the map id $\otimes \bigwedge^{k-i} \beta$ factors through the map $\rho_{i}^{k}$ which sends $\bigwedge^{i} \alpha(u) \wedge v$ to $u \otimes \bigwedge^{k-i} \beta(v)$, while $\tilde{\alpha}_{i+1}^{k}=\alpha_{i+1}^{k} \circ \pi_{i}$, where $\pi_{i}: \bigwedge^{i} M \otimes M \rightarrow \bigwedge^{i+1} M$ is the canonical projection. It is readily seen that diagram (54) commutes, and therefore $\rho_{i}^{k}$ induces an isomorphism $F_{i}^{k} / F_{i+1}^{k} \stackrel{\simeq}{\longrightarrow} \bigwedge^{i} M \otimes \bigwedge^{k-i} P$. This completes the proof. 
12.3. Characteristic varieties and support loci. It has been known for a long time that the characteristic varieties and the Alexander varieties of spaces and groups are intimately related. For instance, it was shown in $[50,19]$ that $\mathscr{V}_{1}(G)=\mathscr{Y}_{1}(G)$ and $\mathscr{W}_{1}(G)=\mathscr{Z}_{1}(G)$, at least away from 1 . Those proofs, based on a change-of-rings spectral sequence argument, are very specific to depth $k=1$ and do not generalize to higher depths. We give here a proof valid in all depths $k \geqslant 1$. The proof is modeled on the proof of [38, Proposition 0.2]; since the argument given there is not complete (a proof of Lemma 12.5 is missing) and not quite in the generality we need (it assumes $G$ is finitely presented), we provide full details.

Theorem 12.6. Let $G$ be a finitely generated group. Then, for all $k \geqslant 1$,

$$
\mathscr{V}_{k}(G)=\operatorname{supp}\left(\bigwedge^{k} B(G) \otimes \mathbb{C}\right)
$$

at least away from the identity $1 \in \mathbb{T}_{G}$.

Proof. Let $B=B(G)$ and $A=A(G)$, viewed as modules over $R=\mathbb{Z}\left[G_{\mathrm{ab}}\right]$, and let $I=I_{\mathbb{Z}}\left(G_{\mathrm{ab}}\right)$ be the augmentation ideal. By (12), we have an exact sequence of $R$ modules, $0 \rightarrow B \rightarrow A \rightarrow I \rightarrow 0$. Fix a maximal ideal $m \in \operatorname{Spec}_{\mathrm{m}}(R)$. Localization is an exact functor; hence, localizing at $\mathrm{m}$ yields an exact sequence of $R_{\mathrm{m}}$-modules,

$$
0 \rightarrow B_{\mathfrak{m}} \rightarrow A_{\mathfrak{m}} \rightarrow I_{\mathfrak{m}} \rightarrow 0 \text {. }
$$

By Lemma 12.5, there is a filtration by $R_{\mathfrak{m}}$-submodules, $\bigwedge^{k} A_{\mathfrak{m}}=F_{0}^{k} \supseteq F_{1}^{k} \supseteq \cdots$, with successive quotients $F_{i}^{k} / F_{i+1}^{k} \cong \bigwedge^{i} B_{\mathfrak{m}} \otimes_{R_{\mathfrak{m}}} \bigwedge^{k-i} I_{\mathfrak{m}}$.

On the other hand, localizing at $\mathrm{m}$ the exact sequence $0 \rightarrow I \rightarrow R \stackrel{\varepsilon}{\rightarrow} \mathbb{Z} \rightarrow 0$, we get the exact sequence $0 \rightarrow I_{\mathfrak{m}} \rightarrow R_{\mathfrak{m}} \rightarrow \mathbb{Z}_{\mathfrak{m}} \rightarrow 0$. Assuming $\mathfrak{m} \neq I$, we have that $\mathbb{Z}_{\mathfrak{m}}=0$, and so $I_{\mathrm{m}}=R_{\mathrm{m}}$. Hence, $\bigwedge^{j} I_{\mathrm{m}}$ is isomorphic to $R_{m}$ if $j=0$ or 1 and is equal to 0 if $j>1$.

Putting things together, we infer the following: for every $k \geqslant 1$ and for every maximal ideal $m \neq I$, we have an exact sequence

$$
0 \longrightarrow \bigwedge^{k} B_{\mathfrak{m}} \longrightarrow \bigwedge^{k} A_{\mathfrak{m}} \longrightarrow \bigwedge^{k-1} B_{\mathfrak{m}} \longrightarrow 0 .
$$

By additivity of supports, it follows that $\operatorname{supp}\left(\bigwedge^{k} A \otimes \mathbb{C}\right)=\operatorname{supp}\left(\bigwedge^{k-1} B \otimes \mathbb{C}\right)$, at least away from $\operatorname{supp}(I)=\{1\}$. On the other hand, by Lemma 12.1, we have that $\operatorname{supp}\left(\bigwedge^{k} A \otimes \mathbb{C}\right)=V\left(\operatorname{Fitt}_{k}(A \otimes \mathbb{C})\right)$. Applying now Lemma 11.2 completes the proof.

The next corollary sharpens a result that goes back to the work of Dwyer and Fried [26], and was further developed in [50, 62, 73]. In all those results, only depth $k=1$ was considered; the novelty here is that we work with arbitrary depth.

Corollary 12.7. Let $G$ be a finitely generated group. For each $k \geqslant 1$, the following conditions are equivalent.

(1) The characteristic variety $\mathscr{V}_{k}(G)$ is a finite subset of $\mathbb{T}_{G}$.

(2) The $\mathbb{C}$-vector space $\bigwedge^{k}(B(G) \otimes \mathbb{C})$ is finite-dimensional. 
Proof. As is well-known (see e.g. [26, 52, 73]), a finitely generated module $M$ over an affine $\mathbb{C}$-algebra $R$ has finite support if and only if $\operatorname{dim}_{\mathbb{C}} M<\infty$. Letting $M=$ $\bigwedge^{k} B(G) \otimes \mathbb{C}$, viewed as a module over the $\mathbb{C}$-algebra $R=\mathbb{C}\left[G_{\mathrm{ab}}\right]$, the claim follows from Theorem 12.6.

Example 12.8. Let $K$ be a tame knot in $S^{3}$, and let $G$ be the fundamental group of the knot complement. Since $G_{\mathrm{ab}}=\mathbb{Z}$, we may identify $\mathbb{T}_{G}=\mathbb{C}^{*}$. The variety $\mathscr{V}_{1}(G)$ consists of 1 , together with the roots of the Alexander polynomial of the knot, $\Delta_{K} \in \mathbb{Z}\left[t^{ \pm 1}\right]$; in particular, $\mathscr{V}_{1}(G)$ is finite. By the above corollary, the $\mathbb{C}$-vector space $B(G) \otimes \mathbb{C}$ is finite-dimensional; in fact, as is well-known, its dimension is equal to $\operatorname{deg} \Delta_{K}$.

12.4. Restricting to the character torus. We now restrict out attention to the identity component of the character group, $\mathbb{T}_{G}^{0}$, and prove analogous results for the restricted characteristic varieties, $\mathscr{W}_{k}(G)=\mathscr{V}^{k}(G) \cap \mathbb{T}_{G}^{0}$, and the corresponding support loci, $\mathscr{Z}_{k}(G)=\operatorname{supp}\left(\bigwedge^{k} B_{\mathbb{Q}}(G) \otimes \mathbb{C}\right)$.

Theorem 12.9. Let $G$ be a finitely generated group. Then, for all $k \geqslant 1$.

$$
\mathscr{W}_{k}(G)=\operatorname{supp}\left(\bigwedge^{k} B_{\mathbb{Q}}(G) \otimes \mathbb{C}\right),
$$

at least away from the identity $1 \in \mathbb{T}_{G}^{0}$.

Proof. Let $B_{Q}=B_{Q}(G) \otimes \mathbb{C}$ and $A_{Q}=A_{Q}(G) \otimes \mathbb{C}$, and let $I_{0}$ be the augmentation ideal of $\mathbb{C}\left[G_{\mathrm{abf}}\right]$. By Lemma 3.4, part (3), we have an an exact sequence of $\mathbb{C}\left[G_{\mathrm{abf}}\right]$-modules, $0 \rightarrow B_{Q} \rightarrow A_{Q} \rightarrow I_{0} \rightarrow 0$. Continuing as in the proof of Theorem 12.6, we find that $\operatorname{supp}\left(\bigwedge^{k} A_{\odot}\right)=\operatorname{supp}\left(\bigwedge^{k-1} B_{\odot}\right)$, at least away from $\{1\}$. On the other hand, by Lemma 12.1, we have that supp $\left(\bigwedge^{k} A_{\mathbb{Q}}\right)=V\left(\operatorname{Fitt}_{k}\left(A_{\odot}\right)\right)$. Applying now Lemma 11.3 completes the proof.

It follows at once from Theorems 12.6 and 12.9 that $\mathscr{Z}_{k}(G)=\mathscr{Y}_{k}(G) \cap \mathbb{T}_{G}^{0}$. Moreover, we have the following corollary, which, in view of Lemma 3.2, part (2), sharpens results from [26, 50, 62, 73].

Corollary 12.10. Let $G$ be a finitely generated group. For each $k \geqslant 1$, the following conditions are equivalent.

(1) The characteristic variety $\mathscr{W}_{k}(G)$ is a finite subset of $\mathbb{T}_{G}^{0}$.

(2) The $\mathbb{Q}$-vector space $\bigwedge^{k} B_{\mathbb{Q}}(G) \otimes \mathbb{Q}$ is finite-dimensional.

Proof. Follows from Theorem 12.9 using the same argument as in the proof of Corollary 12.7, applied this time to the vector space $M=\bigwedge^{k} B_{\mathbb{Q}}(G) \otimes \mathbb{C}$, viewed as a module over the $\mathbb{C}$-algebra $R=\mathbb{C}\left[G_{\text {abf }}\right]$.

As an application, we obtain the following corollary.

Corollary 12.11. Let $G$ be a finitely generated group, and suppose $\mathscr{W}_{1}(G)$ is finite. Then the Chen ranks $\theta_{n}(G)$ vanish for $n \gg 0$. 
Proof. By Corollary 6.8, we have that $\theta_{n}(G)=\operatorname{dim}_{\mathbb{Q}} \operatorname{gr}_{n-2}\left(B_{\mathbb{Q}}(G) \otimes \mathbb{Q}\right)$ for all $n \geqslant 2$. On the other hand, since $\mathscr{W}_{1}(G)$ is finite, Corollary 12.10 implies that $B_{\mathbb{Q}}(G) \otimes \mathbb{Q}$ is finite-dimensional. Hence, the associated graded vector space $\operatorname{gr}\left(B_{\mathbb{Q}}(G) \otimes \mathbb{Q}\right)$ is also finite-dimensional; thus, its graded pieces must vanish in sufficiently high degrees.

\section{Characteristic Varieties In GROUP EXTENSIONS}

We exhibit in this section several relationships between the characteristic varieties of a finitely generated group $G$, of a normal subgroup $K$, and of the quotient group $Q=G / K$.

13.1. Homomorphisms and jump loci. Let $\alpha: G \rightarrow H$ be a homomorphism between two finitely generated groups. Letting $\alpha_{\mathrm{ab}}: G_{\mathrm{ab}} \rightarrow H_{\mathrm{ab}}$ be the induced map on abelianizations, its linear extension to group algebras, $\tilde{\alpha}: \mathbb{C}\left[G_{\mathrm{ab}}\right] \rightarrow \mathbb{C}\left[H_{\mathrm{ab}}\right]$, defines a morphism between the corresponding maximal spectra. Under our previous identifications, this morphism coincides with the induced map on character groups, $\alpha^{*}: \mathbb{T}_{H} \rightarrow \mathbb{T}_{G}$, given by $\alpha^{*}(\rho)(g)=\alpha(\rho(g))$. In general, this morphism may not send $\mathscr{V}_{k}(H)$ to $\mathscr{V}_{k}(G)$, even when $\alpha$ is injective. Here is a simple example.

Example 13.1. Let $F_{n}$ be the free group of rank $n \geqslant 2$, and let $\mathbb{Z}<F_{n}$ be a cyclic subgroup. The inclusion $\iota: \mathbb{Z} \rightarrow F_{n}$ induces a surjective morphism on character tori, $\iota^{*}:\left(\mathbb{C}^{*}\right)^{n} \rightarrow \mathbb{C}^{*}$. This morphism sends $\mathscr{V}_{1}\left(F_{n}\right)=\cdots=\mathscr{V}_{n-1}\left(F_{n}\right)=\left(\mathbb{C}^{*}\right)^{n}$ onto $\mathbb{C}^{*}$ and $\mathscr{V}_{n}\left(F_{n}\right)=\{1\}$ to $\{1\}$; on the other hand, $\mathscr{V}_{1}(\mathbb{Z})=\{1\}$ while $\mathscr{V}_{k}(\mathbb{Z})=\varnothing$ for $k>1$, so the map $\iota^{*}$ does not preserve characteristic varieties, for any depth $k \leqslant n$.

Under certain assumptions, though, the characteristic varieties are preserved. We present now one such situation, and will return to this issue in Theorem 13.3, where a completely different situation will be analyzed.

Suppose $\pi: G \rightarrow Q$ is surjective; then clearly $\pi^{*}: \mathbb{T}_{Q} \rightarrow \mathbb{T}_{G}$ is injective. Moreover, $\pi^{*}$ sends the characteristic varieties of $Q$ to those of $G$. We proved this assertion in [62, Lemma 2.13], starting from the jump loci definition (46) of the characteristic varieties, and using a spectral sequence argument. We give here another, self-contained proof of this result, based on the support loci interpretation from the previous section.

Proposition 13.2 ([62]). Let $G$ be a finitely generated group, and let $\pi: G \rightarrow Q$ be a surjective homomorphism. Then the induced morphism between character groups, $\pi^{*}: \mathbb{T}_{Q} \hookrightarrow \mathbb{T}_{G}$, restricts to embeddings $\mathscr{V}_{k}(Q) \hookrightarrow \mathscr{V}_{k}(G)$ for all $k \geqslant 1$.

Proof. The homomorphism $\pi_{\mathrm{ab}}: G_{\mathrm{ab}} \rightarrow Q_{\mathrm{ab}}$ extends linearly to a ring map, $\tilde{\pi}_{\mathrm{ab}}: R \rightarrow S$, between the (commutative) rings $R=\mathbb{Z}\left[G_{\mathrm{ab}}\right]$ and $S=\mathbb{Z}\left[Q_{\mathrm{ab}}\right]$. Moreover, the map $\pi: G \rightarrow Q$ induces an epimorphism $B(\pi): B(G) \rightarrow B(Q)$ which covers the map $\tilde{\pi}$. The claim now follows from Lemma 12.2 and Theorem 12.6 upon complexifying all these rings and modules and taking supports of exterior powers. 
13.2. Jump loci in ab-exact and abf-exact sequences. Let $K \triangleleft G$ be a normal subgroup, and assume both $G$ and $K$ are finitely generated. The inclusion map $\iota: K \hookrightarrow G$ induces a surjective algebraic morphism between character groups, $\iota^{*}: \mathbb{T}_{G} \rightarrow \mathbb{T}_{K}$, which restricts to a surjective map between the identity components of those groups, $\iota^{*}: \mathbb{T}_{G}^{0} \rightarrow \mathbb{T}_{K}^{0}$. The next theorem shows that, under appropriate triviality assumptions on the monodromy of the resulting extension, these maps preserve the respective characteristic varieties.

Theorem 13.3. Let $1 \rightarrow K \stackrel{\iota}{\rightarrow} G \rightarrow Q \rightarrow 1$ be an exact sequence of finitely generated groups.

(1) If the sequence is ab-exact and $Q$ is abelian, then the map $\iota^{*}: \mathbb{T}_{G} \rightarrow \mathbb{T}_{K}$ restricts to maps $\iota^{*}: \mathscr{V}_{k}(G) \rightarrow \mathscr{V}_{k}(K)$ for all $k \geqslant 1$; furthermore, the map $\iota^{*}: \mathscr{V}_{1}(G) \rightarrow$ $\mathscr{V}_{1}(K)$ is a surjection.

(2) If the sequence is abf-exact and $Q$ is torsion-free abelian, then the map $\iota^{*}: \mathbb{T}_{G}^{0} \rightarrow$ $\mathbb{T}_{K}^{0}$ restricts to maps $\iota^{*}: \mathscr{W}_{k}(G) \rightarrow \mathscr{W}_{k}(K)$ for all $k \geqslant 1$; furthermore, the map $\iota^{*}: \mathscr{W}_{1}(G) \rightarrow \mathscr{W}_{1}(K)$ is a surjection.

Proof. Let $R=\mathbb{Z}\left[K_{\mathrm{ab}}\right]$ and $S=\mathbb{Z}\left[G_{\mathrm{ab}}\right]$. The induced map between Alexander invariants, $B(\iota): B(K) \rightarrow B(G)$, covers the ring map $\tilde{\iota}_{\mathrm{ab}}: R \rightarrow S$ obtained from $\iota_{\mathrm{ab}}: K_{\mathrm{ab}} \rightarrow G_{\mathrm{ab}}$ by linear extension to group rings. Furthermore, $B(\iota)$ may be viewed as the composite $B(K) \rightarrow B(G)_{\iota} \rightarrow B(G)$, where the first arrow is a map of $R$-modules and the second arrow is the identity map of $B(G)$, thought of as a map covering $\tilde{\iota}_{\mathrm{ab}}$. Taking exterior $k$-powers, we may realize the map $\bigwedge^{k} B(\iota)$ as the composite

$$
\bigwedge_{R}^{k} B(K) \longrightarrow \bigwedge_{R}^{k} B(G)_{\iota} \longrightarrow \bigwedge_{S}^{k} B(G) .
$$

To prove claim (1), first note that the map $\iota_{\mathrm{ab}}$ is injective, since the given sequence is ab-exact. Thus, the map $\tilde{\iota}_{\mathrm{ab}}$ is also injective, and the induced map between character groups, $\iota^{*}: \mathbb{T}_{G} \rightarrow \mathbb{T}_{K}$, is surjective. Furthermore, since $Q$ is abelian, Theorem 8.7, part (2) shows that the map $B(K) \rightarrow B(G)$, is an $R$-isomorphism. Therefore, the map $\tilde{\iota}_{\mathrm{ab}}$ restricts to a map $\operatorname{ann}_{R}\left(\bigwedge_{R}^{k} B(K)\right) \rightarrow \operatorname{ann}_{S}\left(\bigwedge_{S}^{k} B(G)\right)$. Applying Theorem 12.6, we obtain a map $\mathscr{V}_{k}(G) \rightarrow \mathscr{V}_{k}(K)$ after tensoring with $\mathbb{C}$ and taking zero-sets. By a previous remark, this map coincides with the restriction of $\iota^{*}$ to $\mathscr{V}_{k}(G)$.

When $k=1$, the map $B(G)_{\iota} \rightarrow B(G)$ is injective; the argument above then shows that the map $\iota^{*}: \mathscr{V}_{1}(G) \rightarrow \mathscr{V}_{1}(K)$ is surjective, and the proof of the first claim is complete.

To prove claim (2), first note that the map $\iota_{\text {abf }}$ is injective, since by assumption our sequence is abf-exact. Thus, the induced morphism, $\iota^{*}: \mathbb{T}_{G}^{0} \rightarrow \mathbb{T}_{K}^{0}$, is surjective. Moreover, Theorem 12.9 insures that $\mathscr{W}_{k}(G)$ coincides, at least away from 1, with the support of the $\mathbb{C}\left[G_{\text {abf }}\right]$-module $\bigwedge^{k}\left(B_{\mathrm{Q}}(G) \otimes \mathbb{C}\right)$. Since $Q$ is torsion-free abelian, Theorem 9.8, part (2) yields a $\mathbb{Z}\left[K_{\text {abf }}\right]$-isomorphism, $B_{\mathbb{Q}}(K) \rightarrow B_{\mathbb{Q}}(G)_{\iota}$. The second claim now follows as above.

It is clear that, in the above theorem, we need to make some triviality assumptions on the action of $Q$ in first homology, for otherwise we may well have $b_{1}(K)>b_{1}(G)$. 
When this happens, the map $\iota^{*}: \mathbb{T}_{G} \rightarrow \mathbb{T}_{K}$ is not surjective, making it unlikely that it would restrict to a surjection from $\mathscr{V}_{1}(G)$ to $\mathscr{V}_{1}(K)$. We illustrate this point with a simple example, and will expand on this issue in $§ 13.4$.

Example 13.4. Let $G=F_{n}$ be a free group of rank $n>1$; as observed previously, $\mathscr{V}_{1}(G)=\mathbb{T}_{G}$ in this case. Now let $\pi: F_{n} \rightarrow \mathbb{Z}_{m}$ be an epimorphism $(m>1)$; then $K=\operatorname{ker}(\pi)$ is isomorphic to $F_{n m-m+1}$. Thus, the inclusion $\iota^{*}: \mathbb{T}_{G} \rightarrow \mathbb{T}_{K}$ is not surjective, and neither is its restriction to the characteristic varieties, $\iota^{*}: \mathscr{V}_{1}(G) \rightarrow \mathscr{V}_{1}(K)$.

Corollary 13.5. Let $1 \rightarrow K \rightarrow G \rightarrow Q \rightarrow 1$ be an ab-exact sequence of finitely generated groups. Suppose $Q$ is abelian and $\mathscr{V}_{1}(G)$ is finite. Then $\operatorname{dim}_{\mathbb{Q}} B(K) \otimes \mathbb{Q}<\infty$ and $\theta_{n}(K)=0$ for $n \gg 0$.

Proof. By Theorem 13.3, part (1), the inclusion $\iota: K \rightarrow G$ induces a surjective morphism, $\iota^{*}: \mathscr{V}_{1}(G) \rightarrow \mathscr{V}_{1}(K)$. Since $\mathscr{V}_{1}(G)$ is a finite set, the same must be true for $\mathscr{V}_{1}(K)$, and the first claim follows from Corollary 12.7. Now note that $\mathscr{W}_{1}(K)=\mathscr{V}_{1}(K) \cap \mathbb{T}_{K}^{0}$ is also finite, and so the second claim follows from Corollary 12.11; alternatively, the second claim follows from the first one and Corollary 6.8.

Corollary 13.6. Let $1 \rightarrow K \rightarrow G \rightarrow Q \rightarrow 1$ be an abf-exact sequence of finitely generated groups. Suppose $Q$ is torsion-free abelian and $\mathscr{W}_{1}(G)$ is finite. Then $\operatorname{dim}_{\mathbb{Q}} B_{\mathbb{Q}}(K) \otimes$ $\mathbb{Q}<\infty$ and $\theta_{n}(K)=0$ for $n \gg 0$.

Proof. This is proved in like fashion: the first claim follows from Theorem 13.3, part (2) and Corollary 12.10, while the second claim follows again from Corollary 12.11 (or from the first one and Corollary 6.8).

13.3. Jump loci in split-exact sequences. For split extensions, Theorem 13.3 admits a slightly more convenient formulation.

Corollary 13.7. Let $1 \rightarrow K \stackrel{\iota}{\rightarrow} G \rightarrow Q \rightarrow 1$ be a split exact sequence of finitely generated groups.

(1) If $Q$ is abelian and acts trivially on $H_{1}(K ; \mathbb{Z})$, then the map $\iota^{*}: \mathbb{T}_{G} \rightarrow \mathbb{T}_{K}$ restricts to maps $\iota^{*}: \mathscr{V}_{k}(G) \rightarrow \mathscr{V}_{k}(K)$ for all $k \geqslant 1$; furthermore, the map $\iota^{*}: \mathscr{V}_{1}(G) \rightarrow$ $\mathscr{V}_{1}(K)$ is a surjection.

(2) If $Q$ is torsion-free abelian and acts trivially on $H_{1}(K ; \mathbb{Q})$, then the map $\iota^{*}: \mathbb{T}_{G}^{0} \rightarrow$ $\mathbb{T}_{K}^{0}$ restricts to maps $\iota^{*}: \mathscr{W}_{k}(G) \rightarrow \mathscr{W}_{k}(K)$ for all $k \geqslant 1$; furthermore, the map $\iota^{*}: \mathscr{W}_{1}(G) \rightarrow \mathscr{W}_{1}(K)$ is a surjection.

Proof. Both claims follow from Theorem 13.3, together with Proposition 8.4 for the first one, and Proposition 9.4, part (2) for the second one.

Here is a large class of examples where Corollary 13.7 applies.

Example 13.8. Let $\Gamma$ be a connected, finite graph, and let $1 \rightarrow N_{\Gamma} \stackrel{\iota}{\rightarrow} G_{\Gamma} \rightarrow \mathbb{Z} \rightarrow 1$ be the ab-exact exact sequence from (44). In [47, Lemma 8.3(i)], it was shown that the 
map $\iota^{*}: \mathbb{T}_{G_{\Gamma}} \rightarrow \mathbb{T}_{N_{\Gamma}}$ restricts to a surjection $\iota^{*}: \mathscr{V}_{1}\left(G_{\Gamma}\right) \rightarrow \mathscr{V}_{1}\left(N_{\Gamma}\right)$, provided $\pi_{1}\left(\Delta_{\Gamma}\right)=0$. Corollary 13.7 recovers this result, without this additional assumption.

13.4. Discussion. For the rest of this section, we discuss the necessity of the assumptions we made in Corollary 13.7, and thus, implicitly, in Theorem 13.3, too. The first example shows why it is necessary to assume that the action of $Q$ on $H_{1}(K ; \mathbb{Z})$ ought to be trivial, even when $Q=\mathbb{Z}$ and $b_{1}(K)=b_{1}(G)$.

Example 13.9. Let $G=\left\langle t, a \mid t a t^{-1}=a^{-1}\right\rangle$ be the fundamental group of the Klein bottle. Then $G=\mathbb{Z} \rtimes_{\varphi} \mathbb{Z}$, where the monodromy automorphism $\varphi$ acts by inversion on the subgroup $K=\mathbb{Z}=\langle a\rangle$, and $G_{\mathrm{ab}}=\mathbb{Z} \oplus \mathbb{Z}_{2}$. The inclusion $\iota: K \rightarrow G$ induces a surjection $\iota^{*}: \mathbb{T}_{G} \rightarrow \mathbb{T}_{K}$, where $\mathbb{T}_{G}=\mathbb{C}^{*} \times\{ \pm 1\}$ and $\mathbb{T}_{K}=\mathbb{C}^{*}$. This map sends $\mathscr{V}_{1}(G)=\{(1,1),(-1,1)\}$ onto $\{ \pm 1\} \subset \mathbb{C}^{*}$, but $\{ \pm 1\}$ is not contained in $\mathscr{V}_{1}(K)=\{1\}$.

The next example shows that the condition requiring $Q$ to be abelian is crucial for Corollary 13.7 to hold, even when $Q$ acts trivially on abelianization.

Example 13.10. Let $P_{n}$ be the Artin pure braid group on $n \geqslant 4$ strands. We then have a split exact sequence, $1 \rightarrow F_{n-1} \stackrel{\iota}{\rightarrow} P_{n} \rightarrow P_{n-1} \rightarrow 1$, with monodromy given by the Artin embedding, $P_{n-1} \hookrightarrow \operatorname{Aut}\left(F_{n-1}\right)$. Since pure braids act trivially on $H_{1}\left(F_{n-1} ; \mathbb{Z}\right)$, the sequence is ab-exact; moreover, both $P_{n}$ and $F_{n-1}$ are finitely generated, though of course $P_{n-1}$ is not abelian. It is known that $\operatorname{dim}\left(\mathscr{V}_{1}\left(P_{n}\right)\right)=2$ (see e.g. [63] and references therein), whereas $\operatorname{dim}\left(\mathscr{V}_{1}\left(F_{n-1}\right)\right)=n-1$. Thus, the morphism $\iota^{*}: \mathbb{T}_{P_{n}} \rightarrow \mathbb{T}_{F_{n-1}}$ does not restrict to a surjection $\mathscr{V}_{1}\left(P_{n}\right) \rightarrow \mathscr{V}_{1}\left(F_{n-1}\right)$.

Remark 13.11. Given an ab-exact sequence as in Corollary 13.7, part (1), the morphisms $\iota^{*}: \mathscr{V}_{k}(G) \rightarrow \mathscr{V}_{k}(K)$ may fail to be surjective for $k>1$. The reason is that exterior powers do not necessarily commute with restriction of scalars, and so the map $\bigwedge_{R}^{k} B(K) \rightarrow$ $\bigwedge_{S}^{k} B(G)$, may fail to be injective.

Computations done in [64, Example 6.5] may be used to show that the phenomenon mentioned in the above remark occurs in the context of Milnor fibrations of complex hyperplane arrangements. This topic will be discussed in more detail in [67]. We give here a different kind of example; we will return to it in $\S 17.3$ in order to illustrate a related point regarding the higher-depth resonance varieties.

Example 13.12. Let $L$ be the link of 4 great circles in $S^{3}$ corresponding to the arrangement of transverse planes through the origin of $\mathbb{R}^{4}$ denoted by $\mathscr{A}(2134)$ in [44, Example 10.1]. The link group, $G=\pi_{1}\left(S^{3} \backslash L\right)$, fits into a split extension, $1 \rightarrow F_{3} \stackrel{\iota}{\rightarrow} G \rightarrow \mathbb{Z} \rightarrow 1$, with monodromy given by a pure braid in $P_{3}$; thus, the extension is ab-exact, and all the hypothesis of Corollary 13.7, part (1) are satisfied. The variety $\mathscr{V}_{1}(G)$ consists of two codimension 1 subtori in $\left(\mathbb{C}^{*}\right)^{4}$, which are sent by $\iota^{*}$ onto $\mathscr{V}_{1}\left(F_{3}\right)=\left(\mathbb{C}^{*}\right)^{3}$, as predicted. On the other hand, $\mathscr{V}_{2}(G)$ consists of two 1-dimensional subtori and a 2-dimensional translated subtorus; thus, its image under $\iota^{*}$ is strictly contained in $\mathscr{V}_{2}\left(F_{3}\right)=\left(\mathbb{C}^{*}\right)^{3}$. 


\section{Part IV. Holonomy and resonance}

\section{Holonomy LiE ALgEBRAS AND FORMALITY PROPERTIES}

In this section we review some basic notions regarding the holonomy Lie algebra and the Malcev Lie algebra of a finitely generated group $G$, and discuss the graded formality and 1-formality properties of such groups.

14.1. The holonomy Lie algebra of a group. Let $G$ be a group such that the maximal torsion-free abelian quotient $G_{\text {abf }}$ is finitely generated. There is then another graded Lie algebra that can be associated to it, besides the ones already mentioned in $\S 5$. This Lie algebra is much easier to understand, in that it uses only information about the cohomology ring of $G$ encoded in the cup-product map $\cup_{G}: H^{1}(G) \wedge H^{1}(G) \rightarrow H^{2}(G)$.

More precisely, let $\mathbf{L}=\operatorname{Lie}\left(G_{\text {abf }}\right)$ be the free Lie algebra on $G_{\text {abf }}$. This is a (positively) graded Lie algebra, with grading given by bracket length. Writing $\mathbf{L}=\bigoplus_{n \geqslant 1} \mathbf{L}_{n}$, we have $\mathbf{L}_{1}=G_{\text {abf }}$ and $\mathbf{L}_{2}=G_{\text {abf }} \wedge G_{\text {abf }}$. Taking the dual of the cup-product map and writing $H^{\vee}:=\operatorname{Hom}(H, \mathbb{Z})$, we obtain the comultiplication map,

$$
\cup_{G}^{\vee}: H^{2}(G)^{\vee} \longrightarrow\left(H^{1}(G) \wedge H^{1}(G)\right)^{\vee} \cong G_{\mathrm{abf}} \wedge G_{\mathrm{abf}}
$$

Following [10, 40, 45, 46, 69], we define the holonomy Lie algebra of $G$, denoted by $\mathfrak{h}(G)$, as the quotient

$$
\mathfrak{h}(G)=\operatorname{Lie}\left(G_{\mathrm{abf}}\right) /\left(\operatorname{im}\left(\cup_{G}^{\vee}\right)\right)
$$

of the free Lie algebra $\mathbf{L}=\operatorname{Lie}\left(G_{\text {abf }}\right)$ by the Lie ideal generated by the image of $\cup_{G}^{\vee}$, viewed as a subgroup of $\mathbf{L}_{2}$. The holonomy Lie algebra inherits a natural grading from the free Lie algebra, which is compatible with the Lie bracket. By construction, $\mathfrak{h}(G)$ is a quadratic Lie algebra: it is generated in degree 1 by $G_{\text {abf }}$, and all the relations are in degree 2. In particular, the derived Lie subalgebra, $\mathfrak{h}(G)^{\prime}$, coincides with $\mathfrak{h}_{\geqslant 2}(G)$. As noted in [69], the projection map $G \rightarrow G / \gamma_{n}(G)$ induces an isomorphism $\mathfrak{h}(G) \stackrel{\simeq}{\longrightarrow}$ $\mathfrak{h}\left(G / \gamma_{n}(G)\right)$ for all $n \geqslant 3$. Consequently, the holonomy Lie algebra of $G$ depends only on its second nilpotent quotient, $G / \gamma_{3}(G)$.

This construction is functorial. Indeed, let $\alpha: G \rightarrow H$ be a homomorphism between two groups as above; then the induced homomorphism $\alpha_{\mathrm{abf}}: G_{\mathrm{abf}} \rightarrow H_{\mathrm{abf}}$ extends to a morphism $\mathbf{L}\left(\alpha_{\text {abf }}\right): \mathbf{L}\left(G_{\text {abf }}\right) \rightarrow \mathbf{L}\left(H_{\text {abf }}\right)$ between the respective free Lie algebras. The map $\alpha$ also induces a morphism between cohomology rings, and thus sends $\operatorname{im}\left(\cup_{G}^{\vee}\right)$ to $\operatorname{im}\left(\cup_{H}^{\vee}\right)$. Consequently, $\mathbf{L}\left(\alpha_{\text {abf }}\right)$ induces a morphism of graded Lie algebras, $\mathfrak{h}(\alpha): \mathfrak{h}(G) \rightarrow \mathfrak{h}(H)$; it is easily checked that $\mathfrak{h}(\beta \circ \alpha)=\mathfrak{h}(\beta) \circ \mathfrak{h}(\alpha)$.

A notable fact about the holonomy Lie algebra is its relationship to the associated graded Lie algebra, as embodied in the next theorem.

Theorem 14.1 ([40, 45, 69]). For every group $G$ such that $G_{\mathrm{abf}}$ is finitely generated, there exists a natural epimorphism of graded Lie algebras, $\Psi: \mathfrak{h}(G) \rightarrow \operatorname{gr}(G)$, which induces 
isomorphisms in degrees 1 and 2 and descends to epimorphisms $\Psi^{(r)}: \mathfrak{h}(G) / \mathfrak{h}(G)^{(r)} \rightarrow$ $\operatorname{gr}\left(G / G^{(r)}\right)$ for all $r \geqslant 2$.

This result is stated and proved in the references cited, in various degrees of generality. Essentially the same proof works in the slightly more general context adopted here.

It follows from the above theorem that the Lie algebra $\mathfrak{h}(G) / \mathfrak{h}(G)^{\prime \prime}$ maps surjectively onto the Chen Lie algebra $\operatorname{gr}\left(G / G^{\prime \prime}\right)$. Thus, if we define the holonomy Chen ranks of $G$ as $\bar{\theta}_{n}(G):=\operatorname{rank}\left(\mathfrak{h}(G) / \mathfrak{h}(G)^{\prime \prime}\right)_{n}$, we have that $\bar{\theta}_{n}(G) \geqslant \theta_{n}(G)$, for all $n \geqslant 1$.

14.2. Graded formality. Suppose now that $G$ is a group with $b_{1}(G)<\infty$, and let $\mathbb{k}$ be a field of characteristic 0 . We may then define in a completely analogous fashion the holonomy Lie algebra of $G$ with coefficients in $\mathbb{k}$, denoted $\mathfrak{h}(G ; \mathbb{k})$, as the free Lie algebra on $H_{1}(G ; \mathbb{K})$, modulo the ideal generated by the image of the comultiplication map, $H_{2}(G ; \mathbb{k}) \rightarrow H_{1}(G ; \mathbb{k}) \wedge H_{1}(G ; \mathbb{k})$.

It is readily seen that $\mathfrak{h}(G ; \mathbb{k})=\mathfrak{h}(G) \otimes \mathbb{k}$, whenever $G_{\text {abf }}$ is finitely generated. Moreover, Theorem 14.1 has the following analogue in this setting.

Theorem 14.2 ([40, 45, 69]). For every group $G$ with $b_{1}(G)<\infty$ there exists a natural epimorphism of graded Lie algebras, $\Psi_{\mathbb{k}}: \mathfrak{h}(G ; \mathbb{k}) \rightarrow \operatorname{gr}(G) \otimes \mathbb{k}$, which induces isomorphisms in degrees 1 and 2 and descends to epimorphisms $\Psi_{\mathbb{k}}^{(r)}: \mathfrak{h}(G ; \mathbb{k}) / \mathfrak{h}(G ; \mathbb{K})^{(r)} \rightarrow$ $\operatorname{gr}\left(G / G^{(r)}\right) \otimes \mathbb{K}$ for all $r \geqslant 2$.

Consequently, we may define the holonomy Chen ranks for groups $G$ with finite first Betti number as $\bar{\theta}_{n}(G):=\operatorname{dim}_{\mathbb{k}}\left(\mathfrak{h}(G ; \mathbb{k}) / \mathfrak{h}(G ; \mathbb{k})^{\prime \prime}\right)_{n}$. When $G_{\text {abf }}$ is finitely generated, these ranks coincide with the ones already defined in $\$ 14.1$.

Following $[69,70]$, we say that a finitely generated group $G$ is graded formal if the map $\Psi_{\mathbb{k}}: \mathfrak{h}(G ; \mathbb{K}) \rightarrow \operatorname{gr}(G) \otimes \mathbb{k}$ is an isomorphism. This condition is equivalent to $\operatorname{gr}(G) \otimes \mathbb{k}$ being a quadratic Lie algebra. As shown in [70, Theorem 5.11], if $K \leqslant G$ is a retract of a graded formal group $G$, then $K$ is also graded formal. The next theorem gives another condition insuring that graded formality is inherited by a subgroup.

Theorem 14.3. Let $G=K \rtimes Q$ be a split extension of finitely generated groups, and assume $Q$ acts trivially on $H_{1}(K ; \mathbb{K})$. If $G$ is graded formal, then $K$ is also graded formal.

Proof. An analogous result was proved in [70, Theorem 5.12], under the more restrictive assumption that $Q$ should act trivially on $K_{\mathrm{ab}}$. Starting from the hypothesis that the map $\Psi_{\mathbb{k}}: \mathfrak{h}(G ; \mathbb{k}) \rightarrow \operatorname{gr}(G) \otimes \mathbb{k}$ is an isomorphism, the proof uses the splitting $\operatorname{gr}(G) \rightarrow \operatorname{gr}(K)$ provided by Falk and Randell's Theorem 7.2 to conclude that the map $\Psi_{\mathbb{k}}: \mathfrak{h}(K ; \mathbb{k}) \rightarrow$ $\operatorname{gr}(K) \otimes \mathbb{k}$ is also an isomorphism.

In our more general setting, Proposition 9.4 insures that $Q$ acts trivially on $K_{\mathrm{abf}}$. Therefore, the same proof works here, using instead the splitting $\operatorname{gr}(G) \otimes \mathbb{K} \rightarrow \operatorname{gr}(K) \otimes \mathbb{k}$ induced by the splitting $\operatorname{gr}^{\complement}(G) \rightarrow \operatorname{gr}^{\complement}(K)$ from Theorem 7.5. 
14.3. Malcev completion. In [57], Quillen associated to every group $G$ a filtered Lie algebra over the rationals, $\mathrm{m}(G)$, called the Malcev Lie algebra of $G$. Let $I=I_{\mathbb{Q}}(G)$ be the augmentation ideal of the group algebra of $G$, and let

$$
\widehat{\mathbb{Q}[G]}=\underset{\leftarrow}{\lim _{n}} \mathbb{Q}[G] / I^{n}
$$

be the completion of $\mathbb{Q}[G]$ with respect to the $I$-adic filtration. The usual Hopf algebra structure on the group algebra extends to the completion, making $\widehat{\mathbb{Q}[G]}$ into a complete Hopf algebra. By definition, $\mathfrak{m}(G)$ is the Lie algebra of primitive elements in $\widehat{\mathbb{Q}[G]}$, endowed with the induced filtration and the (compatible) Lie bracket $[x, y]=x y-y x$. It is readily seen that this construction is functorial.

Let $\operatorname{gr}(\mathfrak{m}(G))$ be the associated graded Lie algebra with respect to the lower central series filtration of $\mathfrak{m}(G)$. Quillen then showed in [56] that

$$
\operatorname{gr}(m(G)) \cong \operatorname{gr}(G) \otimes \mathbb{Q} .
$$

For more on this topic, we refer to [20, 25, 45, 49, 70, 72]. We will only recall here two results which will prove to be useful to us. The first result, proved in [31] and recorded in [20], is a filtered version of a celebrated result of Stallings ([59, Theorem 7.3]).

Theorem 14.4 ([59, 31, 20]). Let $\alpha: G \rightarrow H$ be a homomorphism that induces an isomorphism $H_{1}(G ; \mathbb{Q}) \stackrel{\simeq}{\rightarrow} H_{1}(H ; \mathbb{Q})$ and an epimorphism $H_{2}(G ; \mathbb{Q}) \rightarrow H_{2}(H ; \mathbb{Q})$. Then $\alpha$ induces an isomorphism of filtered Lie algebras, $\mathfrak{m}(G) \stackrel{\simeq}{\longrightarrow} \mathfrak{m}(H)$, and thus, and isomorphism of graded Lie algebras, $\operatorname{gr}(G) \otimes \mathbb{Q} \stackrel{\simeq}{\longrightarrow} \operatorname{gr}(H) \otimes \mathbb{Q}$.

Let $I$ be the augmentation ideal of the ring $R=\mathbb{Q}\left[G_{\mathrm{ab}}\right]$. The second result, proved in [25, Proposition 5.4], relates the $I$-adic completion of the rationalized Alexander invariant, $B(G) \otimes \mathbb{Q}$, viewed as a module over $\widehat{R}$, to the Malcev Lie algebra of $G$. For a subset $\mathfrak{a} \subset \mathfrak{m}(G)$, we denote by $\overline{\mathfrak{a}}$ its closure in the topology defined by the filtration on $\mathfrak{m}(G)$.

Theorem 14.5 ([25]). For a finitely generated group $G$, there is a filtration-preserving

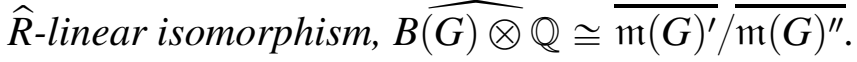

The following is an immediate corollary of the above two theorems.

Corollary 14.6. Let $\alpha: G \rightarrow H$ be a homomorphism between two finitely generated groups. Suppose $\alpha$ induces an isomorphism $H_{1}(G ; \mathbb{Q}) \stackrel{\simeq}{\longrightarrow} H_{1}(H ; \mathbb{Q})$ and an epimorphism $\mathrm{H}_{2}(G ; \mathbb{Q}) \rightarrow \mathrm{H}_{2}(H ; \mathbb{Q})$. Then $\alpha$ induces a filtration-preserving isomorphism

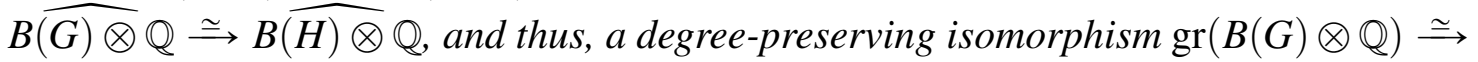
$\operatorname{gr}(B(H) \otimes \mathbb{Q})$.

In particular, as noted in [20], if $G$ is finitely generated, $K \leqslant G$ has finite index, and the inclusion map $\iota: K \rightarrow G$ induces an isomorphism on $H_{1}(-; \mathbb{Q})$, then it also induced an isomorphism between completed Alexander invariants (over $\mathbb{Q}$ ). 
14.4. The 1-formality property. A finitely generated group $G$ is said to be 1-formal if $\mathfrak{m}(G)$ is isomorphic (as a filtered Lie algebra) to $\widehat{h(G ; \mathbb{Q}})$, the completion of the rational holonomy Lie algebra of $G$ with respect to its lower central series filtration.

Now let $\Psi_{\mathbb{Q}}: \mathfrak{h}(G ; \mathbb{Q}) \rightarrow \operatorname{gr}(G) \otimes \mathbb{Q}$ and $\Psi_{\mathbb{Q}}^{(r)}: \mathfrak{h}(G ; \mathbb{Q}) / \mathfrak{h}(G ; \mathbb{Q})^{(r)} \rightarrow \operatorname{gr}\left(G / G^{(r)}\right) \otimes \mathbb{Q}$ be the functorial morphisms of graded Lie algebras from Theorem 14.2. If $G$ is 1 -formal group, then all these morphisms are isomorphisms; this basic result follows from [57] and [45], respectively (see also [49, 69, 70]). Consequently, $G$ is 1-formal if and only if $G$ is graded formal and $\mathrm{m}(G)$ is isomorphic to the completion of its associated graded Lie algebra ([70]). It is still much of an open question as to when the 1-formality property propagates through group extensions. Here is one instance when formality is inherited by (normal) subgroups.

Theorem 14.7 ([21]). Let $K \triangleleft G$ be a normal subgroup. Suppose $G$ is 1-formal, the quotient $Q=G / K$ is finite, and $Q$ acts trivially on $H_{1}(K ; \mathbb{Q})$. Then $K$ is also 1 -formal.

More generally, one may consider the 1-formality problem for short exact sequences of the form $1 \rightarrow K \rightarrow G \rightarrow Q \rightarrow 1$, where both $G$ and $K$ are finitely generated groups. If $K$ is 1-formal, the group $G$ does not have to be 1 -formal, as illustrated by the Heisenberg group $G=\mathbb{Z}^{2} \rtimes \mathbb{Z}$ (see Example 17.4). Likewise, if $G$ is 1 -formal, the group $K$ need not be 1-formal; in fact, $K$ is need not be 1 -formal, even if $Q=G / K$ is finite, see for instance [21, 63]. On the other hand, we have the following positive result.

Theorem 14.8 ([70]). Let $G$ be a 1-formal group, and let $K \triangleleft G$ be a normal subgroup. If $K$ is a retract of $G$, then $K$ is also 1-formal.

\section{Infinitesimal Alexander inVARIANTs AND the BGG correspondence}

We now use the holonomy Lie algebra to construct a graded module over a symmetric algebra which can be viewed as the infinitesimal version of the Alexander invariant. Using the BGG correspondence, we also construct an infinitesimal version of the Alexander module, and relate the two modules via a Crowell-type exact sequence.

15.1. Infinitesimal Alexander invariant. Let $G$ be a group and assume that $G_{\text {abf }}$ is finitely generated. We will denote by $\operatorname{Sym}\left(G_{\text {abf }}\right)$ the symmetric algebra on this free abelian group of finite rank. Note that $\operatorname{Sym}\left(G_{\mathrm{abf}}\right)$ is naturally isomorphic to $\operatorname{gr}\left(\mathbb{Z}\left[G_{\mathrm{abf}}\right]\right)$. In concrete terms, if we identify $G_{\text {abf }}$ with $\mathbb{Z}^{r}$, where $r=b_{1}(G)$, then $\operatorname{Sym}\left(G_{\text {abf }}\right)$ gets identified with the polynomial ring $\mathbb{Z}\left[x_{1}, \ldots, x_{r}\right]$.

A homomorphism $\alpha: G \rightarrow H$ between two groups as above induces a homomorphism $\alpha_{\mathrm{abf}}: G_{\mathrm{abf}} \rightarrow H_{\mathrm{abf}}$, which extends to a ring map, $\tilde{\alpha}_{\mathrm{abf}}: \operatorname{Sym}\left(G_{\mathrm{abf}}\right) \rightarrow \operatorname{Sym}\left(H_{\mathrm{abf}}\right)$. If we indentify these symmetric algebras with the corresponding polynomial rings, the map $\tilde{\alpha}_{\text {abf }}$ is the linear change of variables defined by the matrix of $\alpha_{\text {abf }}$. Consequently, if $\alpha_{\text {abf }}$ is injective (respectively, surjective), then $\tilde{\alpha}_{\text {abf }}$ is also injective (respectively, surjective).

Now let $\mathfrak{h}(G)$ be the holonomy Lie algebra of $G$. Following the approach from [45] (see also $[22,51,53,68,71]$ ), we define the infinitesimal Alexander invariant of $G$ to be 
the quotient group

$$
\mathfrak{B}(G):=\mathfrak{h}(G)^{\prime} / \mathfrak{h}(G)^{\prime \prime},
$$

viewed as a graded module over $\operatorname{Sym}\left(G_{\mathrm{abf}}\right)$. The module structure comes from the exact sequence

$$
0 \longrightarrow \mathfrak{h}(G)^{\prime} / \mathfrak{h}(G)^{\prime \prime} \longrightarrow \mathfrak{h}(G) / \mathfrak{h}(G)^{\prime \prime} \longrightarrow \mathfrak{h}(G) / \mathfrak{h}(G)^{\prime} \longrightarrow 0
$$

via the adjoint action of $\mathfrak{h}(G) / \mathfrak{h}(G)^{\prime}=\mathfrak{h}_{1}(G)=G_{\text {abf }}$ on $\mathfrak{h}(G)^{\prime} / \mathfrak{h}(G)^{\prime \prime}$ given by $g \cdot \bar{x}=$ $\overline{[g, x]}$ for $g \in \mathfrak{h}_{1}(G)$ and $x \in \mathfrak{h}(G)^{\prime}$, and with the grading inherited from the one on $\mathfrak{h}(G)$. When $G$ admits a finite, commutator-relators presentation, this module is isomorphic to the "linearization" of $B(G)$, cf. [45, Proposition 9.3].

The above construction is functorial. More precisely, if $\alpha: G \rightarrow H$ is a homomorphism between two groups as above, then $\alpha$ induces a morphism of graded Lie algebras, $\mathfrak{h}(\alpha): \mathfrak{h}(G) \rightarrow \mathfrak{h}(H)$, which preserves the respective derived series. Hence, the restriction $\mathfrak{h}^{\prime}(\alpha): \mathfrak{h}(G)^{\prime} \rightarrow \mathfrak{h}(H)^{\prime}$ induces a map $\mathfrak{B}(\alpha): \mathfrak{B}(G) \rightarrow \mathfrak{B}(H)$. A routine check shows that $\mathfrak{B}(\alpha)$ is a morphism of modules covering the ring map $\tilde{\alpha}_{\mathrm{abf}}: \operatorname{Sym}\left(G_{\mathrm{abf}}\right) \rightarrow \operatorname{Sym}\left(H_{\mathrm{abf}}\right)$, and that $\mathfrak{B}(\beta \circ \alpha)=\mathfrak{B}(\beta) \circ \mathfrak{B}(\alpha)$.

Denoting by $\mathfrak{B}(H)_{\alpha}$ the module obtained from $\mathfrak{B}(H)$ by restriction of scalars along $\tilde{\alpha}_{\text {abf }}$, we may view the map $\mathfrak{B}(\alpha)$ as the composite $\mathfrak{B}(G) \rightarrow \mathfrak{B}(H)_{\alpha} \rightarrow \mathfrak{B}(H)$, where the first arrow is a $\operatorname{Sym}\left(G_{\text {abf }}\right)$-linear map and the second arrow is the identity map of $\mathfrak{B}(H)$, thought of as covering the ring map $\tilde{\alpha}_{\text {abf }}$.

Consider now the canonical element, $\omega_{G} \in G_{\mathrm{abf}}^{\vee} \otimes G_{\mathrm{abf}}$; by definition, this tensor corresponds to the identity automorphism of $G_{\text {abf }}$ under the tensor-hom adjunction. Identifying $G_{\text {abf }}^{\vee}$ with $H^{1}(G ; \mathbb{Z})$ and $G_{\text {abf }}$ with the degree 1 piece of $S=\operatorname{Sym}\left(G_{\text {abf }}\right)$, multiplication by $\omega_{G}$ in the graded ring $H^{\bullet}(G ; \mathbb{Z}) \otimes S$ restricts to a $S$-linear map, $\cdot \omega_{G}: H^{1}(G ; \mathbb{Z}) \otimes S \rightarrow$ $H^{2}(G ; \mathbb{Z}) \otimes S$. By analogy with $(11)$, we define the infinitesimal Alexander module of $G$ to be the cokernel of the $S$-dual of this map,

$$
\mathfrak{A}(G):=\operatorname{coker}\left(\left(\cdot \omega_{G}\right)^{*}: H^{2}(G ; \mathbb{Z})^{\vee} \otimes S \longrightarrow G_{\mathrm{abf}} \otimes S\right) .
$$

If $\alpha: G \rightarrow H$ is a group homomorphism, it is readily seen that $\left(\alpha_{\mathrm{abf}}^{\vee} \otimes \tilde{\alpha}_{\mathrm{abf}}\right) \circ$ $\left(\cdot \omega_{G}\right)^{*}=\left(\cdot \omega_{H}\right)^{*} \circ\left(H^{2}(\alpha)^{\vee} \otimes \tilde{\alpha}_{\mathrm{abf}}\right)$. Consequently, $\alpha$ induces a morphism of modules, $\mathfrak{A}(\alpha): \mathfrak{A}(G) \rightarrow \mathfrak{A}(H)$, which covers the ring map $\tilde{\alpha}_{\mathrm{abf}}$.

15.2. The BGG correspondence. All these notions may be extended to the more general context of groups $G$ with $b_{1}(G)<\infty$, provided we work over a field $\mathbb{k}$ of characteristic 0. Specifically, let us define the infinitesimal Alexander invariant with coefficients in $\mathbb{k}$ as the quotient

$$
\mathfrak{B}(G ; \mathbb{k})=\mathfrak{h}(G ; \mathbb{k})^{\prime} / \mathfrak{h}(G ; \mathbb{k})^{\prime \prime},
$$

viewed as a graded module over the $\mathbb{k}$-algebra $\operatorname{Sym}\left(H_{1}(G ; \mathbb{k})\right)$. By a previous remark, when $G_{\text {abf }}$ is finitely generated we have that $\mathfrak{h}(G ; \mathbb{k})=\mathfrak{h}(G) \otimes \mathbb{k}$, and so $\mathfrak{B}(G ; \mathbb{k})=$ 
$\mathfrak{B}(G) \otimes \mathbb{k}$. In the particular case when $G$ itself is finitely generated, the module $\mathfrak{B}(G ; \mathbb{C})$ coincides with the Koszul module introduced in [53], and further studied in [1, 2, 3].

We write $H^{\bullet}=H^{\bullet}(G ; \mathbb{k})$ for the cohomology algebra of $G$ with coefficients in $\mathbb{k}$, and $H_{i}=H_{i}(G ; \mathbb{k})$ for the dual $\mathbb{k}$-vector spaces. So let $S=\operatorname{Sym}\left(H_{1}\right)$ be the symmetric $\mathbb{k}$-algebra on $H_{1}$, viewed as the coordinate ring of $H^{1}$. Let $E^{\bullet}=\bigwedge H^{1}$ be the exterior algebra on $H^{1}$; the identification $E^{1}=H^{1}$ extends to a map of graded rings, $E \rightarrow H$, which turns $H$ into an $E$-module. The Bernstein-Gelfand-Gelfand correspondence (see $[28, \S 7 \mathrm{~B}])$ yields a cochain complex of free $S$-modules,

$$
\left(H \otimes_{\mathbb{k}} S, \delta\right): H^{0} \otimes_{\mathbb{K}} S \stackrel{\delta_{H}^{0}}{\longrightarrow} H^{1} \otimes_{\mathbb{K}} S \stackrel{\delta_{H}^{1}}{\longrightarrow} H^{2} \otimes_{\mathbb{K}} S \longrightarrow \cdots .
$$

Upon identifying $H_{1}$ with $S_{1}$, the differentials in (68) are given by multiplication by the canonical element, $\omega \in H^{1} \otimes_{\mathbb{k}} H_{1}$. For $i \geqslant 1$, let $\partial_{i}^{H}: H_{i} \otimes_{\mathbb{k}} S \rightarrow H_{i-1} \otimes_{\mathbb{k}} S$ be the map $S$-dual to $\delta_{H}^{i-1}$. Much as before, we define the infinitesimal Alexander module of $G$ over $\mathbb{R}$ to be the $S$-module

$$
\mathfrak{A}(G, \mathbb{k}):=\operatorname{coker}\left(\partial_{2}^{H}\right) .
$$

Clearly, $\mathfrak{A}(G ; \mathbb{k})=\mathfrak{A}(G) \otimes \mathbb{k}$, provided $G_{\text {abf }}$ is finitely generated.

Finally, if $\alpha: G \rightarrow H$ is a homomorphism, we define as before morphisms $\mathfrak{B}(\alpha ; \mathbb{K})$ and $\mathfrak{A}(\alpha ; \mathbb{K})$ covering the ring map $\tilde{\alpha}_{\text {abf }}: \operatorname{Sym}\left(H_{1}(G ; \mathbb{k})\right) \rightarrow \operatorname{Sym}\left(H_{1}(H ; \mathbb{K})\right)$. When $G_{\text {abf }}$ and $H_{\text {abf }}$ are finitely generated, $\mathfrak{B}(\alpha ; \mathbb{k})=\mathfrak{B}(\alpha) \otimes \mathbb{K}$ and $\mathfrak{A}(\alpha ; \mathbb{k})=\mathfrak{A}(\alpha) \otimes \mathbb{k}$.

15.3. An infinitesimal Crowell exact sequence. Pursuing our analogy with the classical theory of Alexander invariants and modules, we now set up the infinitesimal counterpart (over a field $\mathbb{k}$ of characteristic 0) of Crowell's exact sequence (12).

Theorem 15.1. Let $G$ be a group with $b_{1}(G)<\infty$. Set $S=\operatorname{Sym}\left(H_{1}(G ; \mathbb{K})\right)$, and let $\mathfrak{I}(G ; \mathbb{k})$ be the maximal ideal of $S$ at 0 . There is then a natural exact sequence of $S$ modules,

$$
0 \longrightarrow \mathfrak{B}(G ; \mathbb{k}) \longrightarrow \mathfrak{A}(G ; \mathbb{k}) \longrightarrow \mathfrak{I}(G ; \mathbb{k}) \longrightarrow 0 .
$$

Proof. Writing $H_{1}=H_{1}(G ; \mathbb{k})$, we view $\mathfrak{B}=\mathfrak{B}(G ; \mathbb{k})$ as an $S$-module. As noted in $\S 15.2$, the BGG correspondence produces cochain complexes of free $S$-modules, $\left(H \otimes_{\mathbb{k}}\right.$ $\left.S, \delta_{H}\right)$ and $\left(E \otimes_{\mathbb{k}} S, \delta_{E}\right)$. Dualizing these complexes and setting $E_{i}=\left(E^{i}\right)^{\vee}$ and $\partial_{i+1}=$ $\left(\delta^{i}\right)^{*}$, the functoriality of the BGG correspondence yields the commuting diagram

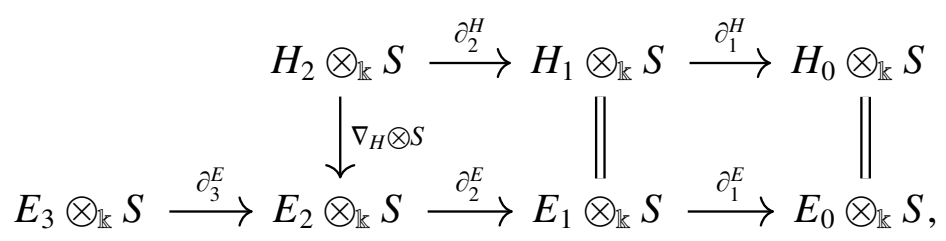

where the bottom row is the beginning of the standard Koszul complex, while $\nabla_{H}: H_{2} \rightarrow$ $E_{2}=H_{1} \wedge H_{1}$ is the comultiplication map $\cup_{G}^{\vee} \otimes \mathbb{K}$ from (60). As shown in [45, Theorem 
6.2], the $S$-module $\mathfrak{B}$ admits the presentation

$$
\left(E_{3} \oplus H_{2}\right) \otimes_{\mathbb{k}} S \stackrel{\partial_{3}^{E}+\nabla_{G} \otimes S}{\longrightarrow} E_{2} \otimes_{\mathbb{k}} S \longrightarrow \mathfrak{B} \longrightarrow 0 .
$$

By definition, $\mathfrak{A}=\mathfrak{A}(G ; \mathbb{k})$ is equal to $\operatorname{coker}\left(\partial_{2}^{H}\right)$. We infer from (71) that the Koszul differential $\partial_{2}^{E}$ descends to a map $\mathfrak{B} \rightarrow \mathfrak{A}$. Noting that $E_{0} \otimes_{\mathbb{K}} S=S$, we may identify $\mathfrak{I}(G ; \mathbb{k})$ with $\operatorname{im}\left(\partial_{1}^{E}\right)$. A diagram chase now yields the exact sequence (70).

Finally, let $\alpha: G \rightarrow H$ be a group homomorphism. It is readily checked that the restriction of $\mathfrak{A}(\alpha ; \mathbb{k})$ to $\mathfrak{B}(G ; \mathbb{K})$ coincides with $\mathfrak{B}(\alpha ; \mathbb{k})$, and induces the map $\tilde{\alpha}_{\text {abf }}$ on augmentation ideals. This verifies the naturality of (70), and completes the proof.

15.4. Chen ranks and 1-formality. Recall we defined in $\$ 14.2$ the holonomy Chen ranks of $G$ as $\bar{\theta}_{n}(G)=\operatorname{dim}_{\mathbb{k}}\left(\mathfrak{h}(G ; \mathbb{k}) / \mathfrak{h}(G ; \mathbb{k})^{\prime \prime}\right)_{n}$, where $\mathbb{k}$ is a field of characteristic 0 . The proof of [68, Proposition 8.1]—adapted to our slightly more general setting-shows that the following infinitesimal version of Massey's correspondence holds.

Proposition 15.2 ([68]). Let $G$ be a group with $b_{1}(G)<\infty$. Then

$$
\bar{\theta}_{n}(G)=\operatorname{dim}_{\mathbb{K}} \mathfrak{B}_{n-2}(G ; \mathbb{K}), \text { for all } n \geqslant 2 \text {. }
$$

It follows from Theorem 14.2 that the Chen ranks $\theta_{n}(G)$ satisfy the inequality $\theta_{n}(G) \leqslant$ $\bar{\theta}_{n}(G)$. As we shall see below, when the group $G$ is 1 -formal, this becomes an equality for all $n$. The key result towards establishing this fact (proved in [25, Theorem 5.6] and further enhanced in [68]) uses the 1-formality hypothesis to construct a functorial isomorphism between the Alexander invariant and its infinitesimal version, at the level of completions.

Theorem 15.3 ([25]). Let $G$ be a 1-formal group. There is then a natural, filtrationpreserving isomorphism of completed modules, $\overline{B(G) \otimes \mathbb{Q}} \cong \mathfrak{B ( G ) \otimes \mathbb { Q }}$.

Passing to associated graded modules, we obtain as an immediate corollary the following result.

Corollary 15.4. If $G$ is 1-formal, then $\operatorname{gr}(B(G) \otimes \mathbb{Q}) \cong \mathfrak{B}(G) \otimes \mathbb{Q}$, as graded modules over $\operatorname{gr}\left(\mathbb{Q}\left[G_{\mathrm{ab}}\right]\right) \cong \operatorname{Sym}\left(H_{1}(G ; \mathbb{Q})\right)$.

The next corollary was first proved in [45, Theorem 4.2]; we provide here a short proof, based on the above results.

Corollary 15.5 ([45]). If $G$ is 1 -formal, then $\theta_{n}(G)=\bar{\theta}_{n}(G)=\operatorname{dim}_{\mathbb{Q}} \mathfrak{B}_{n-2}(G ; \mathbb{Q})$ for all $n \geqslant 2$.

Proof. The classical Massey correspondence, as summarized in formula (39), implies that $\theta_{n}(G)$ is equal to $\operatorname{dim}_{\mathbb{Q}} \operatorname{gr}_{n-2}(B(G) \otimes \mathbb{Q})$. In turn, Corollary 15.4 allows us to replace the dimension of this vector space by the dimension of $\mathfrak{B}_{n-2}(G ; \mathbb{Q})$, which we know from Proposition 15.2 is equal to $\bar{\theta}_{n}(G)$. 


\section{RESONANCE VARIETIES}

The resonance varieties of a group are a different kind of jump loci, built solely from cohomological information in low degrees. In this section, we relate these varieties to the support loci of the infinitesimal Alexander invariant, and discuss some of their properties.

16.1. A stratification of the first cohomology group. Let $G$ be a group, and let $H^{\bullet}=$ $H^{\bullet}(G ; \mathbb{C})$ be its cohomology algebra over $\mathbb{C}$. For our purposes here, we will only consider the truncated algebra $H^{\leqslant 2}$; moreover, we will assume that $b_{1}(G)=\operatorname{dim}_{\mathbb{C}} H^{1}$ is finite. For each element $a \in H^{1}$, we have $a^{2}=0$, and so left-multiplication by $a$ defines a cochain complex,

$$
\left(H, \delta_{a}\right): H^{0} \stackrel{\delta_{a}^{0}}{\longrightarrow} H^{1} \stackrel{\delta_{a}^{1}}{\longrightarrow} H^{2},
$$

with differentials $\delta_{a}^{i}(u)=a \cdot u$ for $u \in H^{i}$. It is readily checked that the specialization of the cochain complex (68) at $a$ coincides with (74); see for instance [19, 65].

The resonance varieties witness the extent to which this complex fails to be exact in the middle. More precisely, for each $k \geqslant 1$, the depth $k$ resonance variety of $G$ is defined as

$$
\mathscr{R}_{k}(G):=\left\{a \in H^{1} \mid \operatorname{dim}_{\mathbb{C}} H^{1}\left(H, \delta_{a}\right) \geqslant k\right\} .
$$

These sets are homogeneous algebraic subvarieties of the affine space $H^{1}=H^{1}(G ; \mathbb{C})$. Clearly, $0 \in \mathscr{R}_{k}(G)$ if and only if $k \leqslant b_{1}(G)$; in particular, $\mathscr{R}_{1}(G)=\varnothing$ if and only if $b_{1}(G)=0$. Furthermore, we have a descending filtration,

$$
H^{1}(G ; \mathbb{C}) \supseteq \mathscr{R}_{1}(G) \supseteq \mathscr{R}_{2}(G) \supseteq \cdots \supseteq \mathscr{R}_{r}(G) \supseteq \mathscr{R}_{r+1}(G)=\varnothing,
$$

where $r=b_{1}(G)$. A linear subspace $U \subset H^{1}$ is said to be isotropic if the restriction of $\cup_{G}: H^{1} \wedge H^{1} \rightarrow H^{2}$ to $U \wedge U$ vanishes; that is, $a b=0$ for all $a, b \in U$. As noted in [65, Lemma 2.2], the variety $\mathscr{R}_{k}(G)$ contains every isotropic subspace of $H^{1}$ whose dimension is at most $k+1$; moreover, $\mathscr{R}_{1}(G)$ is the union of all isotropic planes in $H^{1}$.

The following (well-known) lemma shows that the resonance varieties are determinantal varieties of the infinitesimal Alexander module, and thus, Zariski closed subsets of the affine space $H^{1}$. Proofs in various levels of generality have been given, for instance, in $[43,52,65]$. We give here a quick proof, in a slightly greater generality, along the lines of the proof of Lemma 11.2.

Lemma 16.1. Let $G$ be a group with $b_{1}(G)<\infty$. Then, for all $k \geqslant 1$,

$$
\mathscr{R}_{k}(G)=V\left(\operatorname{Fitt}_{k+1}(\mathfrak{A}(G ; \mathbb{C}))\right),
$$

at least away from $0 \in H^{1}(G ; \mathbb{C})$, with equality at 0 for $k<b_{1}(G)$.

Proof. Let $a \in H^{1}$. By the above discussion, we have that $\delta_{H}^{i}(a)=\delta_{a}^{i}$. Thus, $a$ belongs to $\mathscr{R}_{k}(G)$ if and only if rank $\partial_{2}^{H}(a)+\operatorname{rank} \partial_{1}^{H}(a) \leqslant b_{1}(G)-k$. But $H_{0}=\mathbb{C}$ and $\partial_{1}^{H}(a)=0$ if and only if $a=0$. Since $\mathfrak{A}(G, \mathbb{C})=\operatorname{coker}\left(\partial_{2}^{H}\right)$, the lemma follows. 
16.2. Resonance and exterior powers. The next result identifies the depth- $k$ resonance variety of a group as the support locus of the $k$-th exterior power of its infinitesimal Alexander invariant. The result was first proved in [23, Lemma 4.2] for finitely presented groups, using a specialization argument; in fact, the same proof works for finitely generated groups, see [22, Lemma 5.1]. We offer here a completely different proof, in greater generality, using the BGG correspondence and the localization approach from the proof of Theorem 12.6.

Theorem $16.2([23,22])$. Let $G$ be a group with $b_{1}(G)<\infty$. Then

$$
\mathscr{R}_{k}(G)=\operatorname{supp}\left(\bigwedge^{k} \mathfrak{B}(G) \otimes \mathbb{C}\right)
$$

for all $k \geqslant 1$, at least away from $0 \in H^{1}(G ; \mathbb{C})$.

Proof. Let $0 \rightarrow \mathfrak{B} \rightarrow \mathfrak{A} \rightarrow \mathfrak{I} \rightarrow 0$ be the infinitesimal Crowell exact sequence from Theorem 15.1, over $\mathbb{k}=\mathbb{C}$. Localizing at a maximal ideal $m \in \operatorname{Spec}_{\mathrm{m}}(S)$, we obtain an exact sequence of $S_{\mathfrak{m}}$-modules, $0 \rightarrow \mathfrak{B}_{\mathfrak{m}} \rightarrow \mathfrak{A}_{\mathfrak{m}} \rightarrow \mathfrak{J}_{\mathfrak{m}} \rightarrow 0$. On the other hand, localizing at $\mathrm{m}$ the exact sequence $0 \rightarrow \mathfrak{I} \rightarrow S \rightarrow \mathbb{C} \rightarrow 0$, we get the exact sequence $0 \rightarrow \mathfrak{I}_{\mathfrak{m}} \rightarrow S_{\mathfrak{m}} \rightarrow \mathbb{C}_{\mathfrak{m}} \rightarrow 0$. Assuming $\mathfrak{m} \neq \mathfrak{I}$, we have that $\mathbb{C}_{\mathfrak{m}}=0$, and so $\mathfrak{I}_{\mathfrak{m}}=S_{\mathfrak{m}}$. Hence, $\bigwedge^{j} \mathfrak{J}_{\mathfrak{m}}$ is isomorphic to $S_{m}$ if $j=0,1$ and is equal to 0 if $j>1$.

Lemma 12.5 yields an exact sequence, $0 \rightarrow \bigwedge^{k+1} \mathfrak{B}_{\mathfrak{m}} \rightarrow \bigwedge^{k+1} \mathfrak{A}_{\mathfrak{m}} \rightarrow \bigwedge^{k} \mathfrak{B}_{\mathfrak{m}} \rightarrow 0$, from which we deduce that $\operatorname{supp}\left(\bigwedge^{k} \mathfrak{A}\right)=\operatorname{supp}\left(\bigwedge^{k-1} \mathfrak{B}\right)$, at least away from $\operatorname{supp}(\mathfrak{J})=$ $\{0\}$. On the other hand, by Lemma 12.1, we have that $\operatorname{supp}\left(\bigwedge^{k} \mathfrak{A}\right)=V\left(\operatorname{Fitt}_{k}(\mathfrak{A})\right)$. Applying now Lemma 16.1 completes the proof.

As a consequence of Theorem 16.2, we infer that the resonance varieties only depend on the holonomy Lie algebra of the group. More precisely, let $G_{1}$ and $G_{2}$ be two groups with finite first Betti number, and suppose that $\mathfrak{h}\left(G_{1} ; \mathbb{C}\right) \cong \mathfrak{h}\left(G_{2} ; \mathbb{C}\right)$, as graded Lie algebras. There is then a linear isomorphism, $H^{1}\left(G_{1} ; \mathbb{C}\right) \cong H^{1}\left(G_{2} ; \mathbb{C}\right)$, restricting to isomorphisms $\mathscr{R}_{k}\left(G_{1}\right) \cong \mathscr{R}_{k}\left(G_{2}\right)$ for all $k \geqslant 1$.

16.3. The Tangent Cone formula. Let us identify the tangent space to the character group $\mathbb{T}_{G}=H^{1}\left(G ; \mathbb{C}^{*}\right)$ with the linear space $H^{1}(G ; \mathbb{C})$. We denote by $\operatorname{TC}_{1}\left(\mathscr{V}_{k}(G)\right)$ the tangent cone at the identity to the characteristic variety $\mathscr{V}_{k}(G)$; clearly, this set coincides with $\mathrm{TC}_{1}\left(\mathscr{W}_{k}(G)\right)$. It is known that $\mathrm{TC}_{1}\left(\mathscr{W}_{k}(G)\right)$ is always a (homogeneous) subvariety of the resonance variety $\mathscr{R}_{k}(G)$. The basic relationship between the characteristic and resonance varieties in the 1 -formal setting is encapsulated in the "Tangent Cone formula" from [25, Theorem A], which we recall next.

Theorem 16.3 ([25]). If $G$ is a 1-formal group, then $\mathrm{TC}_{1}\left(\mathscr{W}_{k}(G)\right)=\mathscr{R}_{k}(G)$, for all $k \geqslant 1$.

Far-reaching generalizations of this theorem are now known, but this is all we will need for our purposes here. 
16.4. Vanishing resonance. Particularly interesting — and important for many applications - is the case when the resonance vanishes. In fact, as shown in [52, Theorem 3.3] and [53, Propositions 2.6 and 2.7], the case when $\mathscr{R}_{1}(G)=\{0\}$ is generic, in a sense that can be made very precise.

The next result generalizes [22, Theorem 5.2], which is only valid in depth $k=1$, and is only proved there for finitely generated groups $G$ (as is [53, Lemma 2.4], too).

Corollary 16.4. Let $G$ be a group with $b_{1}(G)<\infty$. For each $k \geqslant 1$, the following conditions are equivalent.

(1) The resonance variety $\mathscr{R}_{k}(G)$ is empty or equal to $\{0\}$.

(2) The $\mathbb{Q}$-vector space $\bigwedge^{k} \mathfrak{B}(G ; \mathbb{Q})$ is finite-dimensional.

Proof. The claim follows from Theorem 16.2 using the same argument as in the proof of Corollary 12.7, applied this time to the vector space $M=\bigwedge^{k} \mathfrak{B}(G ; \mathbb{C})$, viewed as a module over the $\mathbb{C}$-algebra $S=\operatorname{Sym}\left(H_{1}(G ; \mathbb{C})\right)$, and taking into the account the homogeneity of $\mathscr{R}_{k}(G)$.

Corollary 16.5. Let $G$ be a group with $b_{1}(G)<\infty$, and suppose $\mathscr{R}_{1}(G)=\{0\}$. Then the holonomy Chen ranks $\bar{\theta}_{n}(G)$ vanish for $n \gg 0$.

Proof. By Proposition 15.2, we have that $\bar{\theta}_{n}(G)=\operatorname{dim}_{\mathbb{Q}} \mathfrak{B}_{n-2}(G ; \mathbb{Q})$ for all $n \geqslant 2$. The claim now follows from Corollary 16.4, in the case when $k=1$.

Corollary 16.6. Let $G$ be a 1-formal group, and suppose $\mathscr{R}_{1}(G)=\{0\}$. Then the Chen ranks $\theta_{n}(G)$ vanish for $n \gg 0$.

Proof. Follows at once from Corollaries 15.5 and 16.5.

Remark 16.7. The range where those Chen ranks vanish has been made precise in [1]: If $G$ is 1 -formal, $\mathscr{R}_{1}(G)=\{0\}$, and $b_{1}(G) \geqslant 3$, then $\theta_{n}(G)=0$ for $n \geqslant b_{1}(G)-1$. Generalizations of this result to the setting where the resonance does not necessarily vanish will be given in [3].

Remark 16.8. By [22, Theorem C] and [52, Corollary 6.3], the following holds for any finitely generated group $G$ : If $\mathscr{R}_{1}(G) \subseteq\{0\}$, then $\operatorname{dim}_{\mathbb{Q}} \widehat{B(G) \otimes \mathbb{Q}}<\infty$. The converse is not true in general, but it is valid in the case when $G$ is 1 -formal.

\section{Resonance VARIETIES IN GROUP EXTENSIONS}

In this final section we investigate the behavior of the resonance varieties under certain kinds of group extensions.

17.1. Maps between resonance varieties. Let $\alpha: G \rightarrow H$ be a homomorphism between two finitely generated groups. The induced homomorphism in first cohomology, $\alpha^{*}: H^{1}(H ; \mathbb{C}) \rightarrow H^{1}(G ; \mathbb{C})$, may not preserve the resonance varieties. For instance, take a cyclic subgroup $\mathbb{Z}<F_{n}(n \geqslant 2)$ as in Example 13.1. The inclusion $\iota: \mathbb{Z} \rightarrow F_{n}$ 
induces a surjective morphism in first cohomology, $\iota^{*}: \mathbb{C}^{n} \rightarrow \mathbb{C}$; this morphism sends $\mathscr{R}_{1}\left(F_{n}\right)=\mathbb{C}^{n}$ onto $\mathbb{C}$, which strictly contains $\mathscr{R}_{1}(\mathbb{Z})=\{0\}$. We will further illustrate this phenomenon in Example 17.4.

Nevertheless, the resonance varieties enjoy a partial naturality property similar to the one possessed by the characteristic varieties.

Proposition 17.1 ([46, 63]). Let $G$ be a finitely generated group, and let $\pi: G \rightarrow Q$ be a surjective homomorphism. Then the induced homomorphism, $\pi^{*}: H^{1}(Q, \mathbb{C}) \rightarrow$ $H^{1}(G, \mathbb{C})$, is injective, and restricts to embeddings $\mathscr{R}_{k}(Q) \hookrightarrow \mathscr{R}_{k}(G)$ for all $k \geqslant 1$.

Starting directly from definition (75), a proof for $k=1$ was given in [46, Lemma 5.1], while the general case was proved in [63, Proposition A.1]. Alternatively, the proof of Proposition 13.2 can be readily adapted to this context, with the $\mathbb{Z}\left[G_{\mathrm{ab}}\right]$-module $B(G)$ replaced by the $\operatorname{Sym}\left[G_{\text {abf }}\right]$-module $\mathfrak{B}(G)$, and with $\mathscr{R}_{k}(G)$ defined as in (77).

17.2. Resonance in split-exact sequences. Our main goal in this section is to relate the infinitesimal Alexander invariants and the resonance varieties of a group $G$ to those of a normal subgroup $K$, under suitable hypothesis. We start with the case when $G=K \rtimes G / K$ is a semidirect product.

Theorem 17.2. Let $1 \rightarrow K \stackrel{\iota}{\rightarrow} G \stackrel{\pi}{\rightarrow} Q \rightarrow 1$ be a split exact sequence of finitely generated groups such that $Q$ acts trivially on $H_{1}(K ; \mathbb{Q})$ and $G$ is graded formal. Then,

(1) $0 \longrightarrow \mathfrak{h}(K) \otimes \mathbb{Q} \stackrel{\mathfrak{h}(\iota) \otimes \mathbb{Q}}{\longrightarrow} \mathfrak{h}(G) \otimes \mathbb{Q} \stackrel{\mathfrak{h}(\pi) \otimes \mathbb{Q}}{\longrightarrow} \mathfrak{h}(Q) \otimes \mathbb{Q} \longrightarrow 0$ is a split exact sequence of graded Lie algebras.

(2) Suppose $Q$ is abelian. Then

(a) The map $\mathfrak{B}(\iota): \mathfrak{B}(K) \rightarrow \mathfrak{B}(G)$ gives rise to a $\operatorname{Sym}\left(H_{1}(K ; \mathbb{Q})\right)$-linear isomorphism, $\mathfrak{B}(K) \otimes \mathbb{Q} \stackrel{\simeq}{\longrightarrow} \mathfrak{B}(G), \otimes \mathbb{Q}$.

(b) $\bar{\theta}_{n}(K) \leqslant \bar{\theta}_{n}(G)$ for all $n \geqslant 1$.

(c) The morphism $\iota^{*}: H^{1}(G ; \mathbb{C}) \rightarrow H^{1}(K ; \mathbb{C})$ restricts to maps $\iota^{*}: \mathscr{R}_{k}(G) \rightarrow$ $\mathscr{R}_{k}(K)$ for all $k \geqslant 1$; moreover, the map $\iota^{*}: \mathscr{R}_{1}(G) \rightarrow \mathscr{R}_{1}(K)$ is a surjection.

Proof. Since $K$ is finitely generated and $Q$ acts trivially on $H_{1}(K ; \mathbb{Q})$, Proposition 9.4 insures that the sequence $1 \rightarrow K \rightarrow G \rightarrow Q \rightarrow 1$ is abf-exact; in particular, $\iota_{\mathrm{abf}}$ is injective and $\bar{\theta}_{1}(K)=\operatorname{rank} K_{\mathrm{abf}}$ is less or equal to $\bar{\theta}_{1}(G)=\operatorname{rank} G_{\mathrm{abf}}$. By Corollary 9.5, the given sequence yields a split exact sequence of associated graded $\mathbb{Q}$-Lie algebras, $0 \rightarrow \operatorname{gr}(K) \otimes \mathbb{Q} \rightarrow \operatorname{gr}(G) \otimes \mathbb{Q} \rightarrow \operatorname{gr}(Q) \otimes \mathbb{Q} \rightarrow 0$.

The assumption that $G$ is graded formal means that the natural morphism $\mathfrak{h}(G) \otimes \mathbb{Q} \rightarrow$ $\operatorname{gr}(G) \otimes \mathbb{Q}$ is an isomorphism. By [70, Theorem 5.11], $Q$ is graded formal, since it is a retract of $G$, a graded formal group. Likewise, Theorem 14.3 implies that $K$ is graded formal. Putting things together shows that the sequence from (1) is also a split exact sequence of graded $\mathbb{Q}$-Lie algebras.

Assume now that $Q$ is abelian. We then have $\operatorname{gr}_{n}(Q)=0$ for all $n \geqslant 2$, and so $\mathfrak{h}_{n}(Q) \otimes$ $\mathbb{Q}=0$ for all $n \geqslant 2$. Using part (1), we infer that $\mathfrak{h}(K)^{\prime} \otimes \mathbb{Q} \cong \mathfrak{h}(G)^{\prime} \otimes \mathbb{Q}$. Therefore, we 
also have $\mathfrak{h}(K)^{\prime \prime} \otimes \mathbb{Q} \cong \mathfrak{h}(G)^{\prime \prime} \otimes \mathbb{Q}$. Thus, the induced map, $\mathfrak{B}(K) \otimes \mathbb{Q} \rightarrow \mathfrak{B}(G)_{\iota} \otimes \mathbb{Q}$, is an isomorphism of modules over $\operatorname{Sym}\left(H_{1}(K ; \mathbb{Q})\right)$, and claim $(2 \mathrm{a})$ is established.

By Proposition 15.2, we have that $\bar{\theta}_{n}(G)=\operatorname{dim}_{\mathbb{k}} \mathfrak{B}_{n-2}(G) \otimes \mathbb{Q}$ for $n \geqslant 2$, and likewise for $\bar{\theta}_{n}(K)$. Therefore, claim (2b) for $n \geqslant 2$ follows from part (2a), using the injectivity of the map $\tilde{\iota}_{\mathrm{abf}}: \operatorname{Sym}\left(K_{\mathrm{abf}}\right) \rightarrow \operatorname{Sym}\left(G_{\mathrm{abf}}\right)$ and a reasoning similar to the proof of Theorem 9.8, part (4).

By Theorem 16.2, we have that $\mathscr{R}_{k}(G)=\operatorname{supp}\left(\bigwedge^{k} \mathfrak{B}(G) \otimes \mathbb{C}\right)$ and similarly for $\mathscr{R}_{k}(K)$, at least away from 0 . Claim (2c) now follows from part (2a), in a manner similar to the proof of Theorem 13.3.

Corollary 17.3. Let $1 \rightarrow K \rightarrow G \rightarrow Q \rightarrow 1$ be a split exact sequence of finitely generated groups. Assume $Q$ is abelian and acts trivially on $H_{1}(K ; \mathbb{Q})$, while $G$ is graded formal and $\mathscr{R}_{1}(G) \subseteq\{0\}$. Then,

(1) $\operatorname{dim}_{\mathbb{Q}} \mathfrak{B}(K) \otimes \mathbb{Q}<\infty$ and $\mathscr{R}_{1}(K) \subseteq\{0\}$.

(2) $\bar{\theta}_{n}(K) \leqslant \bar{\theta}_{n}(G)$ for all $n \geqslant 1$ and $\bar{\theta}_{n}(K)=0$ for $n \gg 0$.

Proof. All claims follow directly from Theorem 17.2, part (2), and from Corollaries 16.4 and 16.5.

17.3. Discussion and examples. Let us discuss the necessity of some of the assumptions we made in Theorem 17.2. The extension $1 \rightarrow F_{n} \rightarrow P_{n} \rightarrow P_{n-1} \rightarrow 1(n \geqslant 4)$ from Example 13.10 can be used again to show that in part (2) we need to assume $Q$ to be abelian. In the next example, $Q$ is abelian but acts non-trivially on $H_{1}(K ; \mathbb{Q})$, while $G$ is not graded-formal.

Example 17.4. Let $G$ be the Heisenberg group from Example 5.4 and Remark 8.2. This group can be realized as a split extension of the form $\mathbb{Z}^{2} \rtimes_{\varphi} \mathbb{Z}$, with monodromy given by the matrix $\left(\begin{array}{ll}1 & 1 \\ 0 & 1\end{array}\right)$. The inclusion $\iota: \mathbb{Z}^{2} \hookrightarrow G$ induces a homomorphism $\iota^{*}: H^{1}(G ; \mathbb{C}) \rightarrow$ $H^{1}\left(\mathbb{Z}^{2} ; \mathbb{C}\right)$ which can be identified with the linear map $\iota^{*}: \mathbb{C}^{2} \rightarrow \mathbb{C}^{2}$ with matrix $\left(\begin{array}{ll}0 & 0 \\ 0 & 1\end{array}\right)$. Since $\cup_{G}=0$, we have that $\mathscr{R}_{1}(G)=\mathbb{C}^{2}$; thus, $\iota^{*}$ does not take $\mathscr{R}_{1}(G)$ to $\mathscr{R}_{1}\left(\mathbb{Z}^{2}\right)=\{0\}$. For comparison, though, note that the map $\iota^{*}: \mathbb{T}_{G} \rightarrow \mathbb{T}_{\mathbb{Z}^{2}}$ does take $\mathscr{V}_{1}(G)=\{1\}$ to $\mathscr{V}_{1}\left(\mathbb{Z}^{2}\right)=\{1\}$; the discrepancy is due to the fact that $G$ is not 1-formal.

Given an exact sequence as in Theorem 17.2 (or as in Theorem 17.6 below), the morphisms $\iota^{*}: \mathscr{R}_{k}(G) \rightarrow \mathscr{R}_{k}(K)$ may fail to be surjective for $k>1$. The reason is the same as the one given in Remark 13.11: exterior powers do not necessarily commute with restriction of scalars, and so the map $\bigwedge^{k} \mathfrak{B}(K) \otimes \mathbb{Q} \rightarrow \bigwedge^{k} \mathfrak{B}(G)_{\iota} \otimes \mathbb{Q}$ may fail to be injective for $k>1$. We illustrate this phenomenon with an example.

Example 17.5. Let $G$ be the link group from Example 13.12. As noted previously, this group fits into a split exact and ab-exact sequence, $1 \rightarrow F_{3} \stackrel{\iota}{\rightarrow} G \rightarrow \mathbb{Z} \rightarrow 1$. Moreover, the group $G$ is graded formal, see [69, Theorem 7.6]. Thus, all the hypothesis of Theorem 17.2 are satisfied. Now, as shown in [43, Example 6.3], the variety $\mathscr{R}_{1}(G)$ consists of two hyperplanes in $\mathbb{C}^{4}$, while $\mathscr{R}_{2}(G)$ consists of two lines. It is readily seen that $\iota^{*}$ sends 
$\mathscr{R}_{1}(G)$ onto $\mathscr{R}_{1}\left(F_{3}\right)=\mathbb{C}^{3}$, as predicted; by dimension reasons, though, $\iota^{*}\left(\mathscr{R}_{2}(G)\right)$ is strictly contained in $\mathscr{R}_{2}\left(F_{3}\right)=\mathbb{C}^{3}$.

17.4. Resonance in ab-exact and abf-exact sequences. We now relax the split exactness assumption from the preceding theorem at the price of making a more stringent formality assumption.

Theorem 17.6. Let $1 \rightarrow K \stackrel{\iota}{\rightarrow} G \rightarrow Q \rightarrow 1$ be an exact sequence of groups, and assume the following hold.

(i) Either the sequence if ab-exact and $Q$ is abelian, or the sequence is abf-exact and $Q$ is torsion-free abelian.

(ii) Both $G$ and $K$ are 1-formal.

Then

(1) The map $\mathfrak{B}(\iota): \mathfrak{B}(K) \rightarrow \mathfrak{B}(G)$ gives rise to a $\operatorname{Sym}\left(H_{1}(K ; \mathbb{Q})\right)$-linear isomorphism, $\mathfrak{B}(K) \otimes \mathbb{Q} \stackrel{\simeq}{\longrightarrow} \mathfrak{B}(G)_{\iota} \otimes \mathbb{Q}$.

(2) $\theta_{n}(K) \leqslant \theta_{n}(G)$ for all $n \geqslant 1$.

(3) The morphism $\iota^{*}: H^{1}(G, \mathbb{C}) \rightarrow H^{1}(K, \mathbb{C})$ restricts to maps $\iota^{*}: \mathscr{R}_{k}(G) \rightarrow \mathscr{R}_{k}(K)$ for all $k \geqslant 1$; moreover, the map $\iota^{*}: \mathscr{R}_{1}(G) \rightarrow \mathscr{R}_{1}(K)$ is a surjection.

Proof. Assumption (i) says that the hypothesis of either Theorem 8.7 or Theorem 9.8 are satisfied. In either case, we infer that the map $B(\iota): B(K) \rightarrow B(G)$ gives rise to a $\mathbb{Q}\left[H_{1}(K ; \mathbb{Q})\right]$-linear isomorphism, $B(K) \otimes \mathbb{Q} \rightarrow B(G)_{\iota} \otimes \mathbb{Q}$. On the other hand, the formality assumption (ii) and Corollary 15.4 yield functorial isomorphisms $\operatorname{gr}(B(K)) \otimes$ $\mathbb{Q} \cong \mathfrak{B}(K) \otimes \mathbb{Q}$ and $\operatorname{gr}(B(G)) \otimes \mathbb{Q} \cong \mathfrak{B}(G) \otimes \mathbb{Q}$. Claim (1) readily follows.

Under assumption (i), claim (2) follows directly from Theorem 8.7, part (4) in the abexact case, or from Theorem 9.8, part (4) in the abf-exact case. Alternatively, the claim follows from part (1), using the formality assumption (ii) and Corollary 15.5.

Assumption (i) also says that the hypothesis of Theorem 13.3-from either part (1) or part (2)_are satisfied. In both cases, the map $\iota^{*}: \mathbb{T}_{G} \rightarrow \mathbb{T}_{K}$ restricts to maps $\iota^{*}: \mathscr{W}_{k}(G) \rightarrow$ $\mathscr{W}_{k}(K)$ for all $k \geqslant 1$, with the map $\iota^{*}: \mathscr{W}_{1}(G) \rightarrow \mathscr{W}_{1}(K)$ being a surjection. Taking tangent cones at the identities of $\mathbb{T}_{G}$ and $\mathbb{T}_{K}$, respectively, we infer that the homomorphism $\iota^{*}: H^{1}(G ; \mathbb{C}) \rightarrow H^{1}(K ; \mathbb{C})$ restricts to maps $\mathrm{TC}_{1}\left(\mathscr{W}_{k}(G)\right) \rightarrow \mathrm{TC}_{1}\left(\mathscr{W}_{k}(K)\right)$ for $k \geqslant 2$ and to a surjection $\mathrm{TC}_{1}\left(\mathscr{W}_{1}(G)\right) \rightarrow \mathrm{TC}_{1}\left(\mathscr{W}_{1}(K)\right)$.

Finally, by virtue of our 1-formality assumptions on $G$ and $K$, Theorem 16.3 allows us to replace $\mathrm{TC}_{1}\left(\mathscr{W}_{k}(G)\right)$ with $\mathscr{R}_{k}(G)$ and $\mathrm{TC}_{1}\left(\mathscr{W}_{k}(K)\right)$ with $\mathscr{R}_{k}(K)$ for each $k \geqslant 1$. This proves claim (3).

Corollary 17.7. With the notation and assumptions of Theorem 17.6, suppose $\mathscr{R}_{1}(G) \subseteq$ $\{0\}$. Then,

(1) $\operatorname{dim}_{\mathbb{Q}} \mathfrak{B}(K) \otimes \mathbb{Q}<\infty$ and $\mathscr{R}_{1}(K) \subseteq\{0\}$.

(2) $\theta_{n}(K) \leqslant \theta_{n}(G)$ for all $n \geqslant 1$ and $\theta_{n}(K)=0$ for $n \gg 0$.

Proof. All claims follow from the theorem and from Corollaries 16.4 and 16.6. 
We conclude with a general class of examples where Theorem 17.6 applies.

Example 17.8. For a finite, connected graph $\Gamma$, the right-angled Artin group $G_{\Gamma}$ is always 1-formal (see [46]), whereas the Bestvina-Brady group $N_{\Gamma}$ is 1-formal whenever $\pi_{1}\left(\Delta_{\Gamma}\right)=0$ (see [47]), or, more generally, $H_{1}\left(\Delta_{\Gamma} ; \mathbb{Q}\right)=0$ (see [48]). When $\Delta_{\Gamma}$ is simplyconnected, Theorem 17.6, part (2) recovers Lemma 8.3(ii) from [47]. In the broader setting when $b_{1}\left(\Delta_{\Gamma}\right)=0$ yet $\pi_{1}\left(\Delta_{\Gamma}\right) \neq 0$ (for instance, when $\Gamma$ is the 1-skeleton of a flag triangulation of $\mathbb{R} P^{2}$ ), our theorem still applies, although in this case $N_{\Gamma}$ is finitely generated yet not finitely presented.

\section{REFERENCES}

[1] M. Aprodu, G. Farkas, S. Papadima, C. Raicu, J. Weyman, Topological invariants of groups and Koszul modules, arXiv: 1806.01702v1.1.8, 15.2, 16.7

[2] M. Aprodu, G. Farkas, S. Papadima, C. Raicu, J. Weyman, Koszul modules and Green's Conjecture, Invent. Math. 218 (2019), 657-720. MR4022070 1.8, 15.2

[3] M. Aprodu, G. Farkas, C. Raicu, A. Suciu, An effective proof of the Chen ranks conjecture, draft (2021). 1.8, 15.2, 16.7

[4] H. Bass, A. Lubotzky, Linear-central filtrations on groups, in: The mathematical legacy of Wilhelm Magnus: groups, geometry and special functions (Brooklyn, NY, 1992), 45-98, Contemp. Math., vol. 169, Amer. Math. Soc., Providence, RI, 1994. MR1292897 1.2, 5.2, 5.2, 5.2, 5.1, 5.3

[5] P. Bellingeri, S. Gervais, On p-almost direct products and residual properties of pure braid groups of nonorientable surfaces, Alg. Geom. Topol. 16 (2016) 547-568. MR3470709 1.4, 5.3, 7.3, 7.3, 7.8

[6] P. Bellingeri, S. Gervais, J. Guaschi, Exact sequences, lower central series and representations of surface braid groups, arXiv: 1106.4982v1.7.1

[7] M. Bestvina, N. Brady, Morse theory and finiteness properties of groups, Invent. Math. 129 (1997), no. 3, 445-470. MR1465330 1.5, 8.5

[8] K.S. Brown, Cohomology of groups, Corrected reprint of the 1982 original. Graduate Texts in Mathematics, vol. 87, Springer-Verlag, New York, 1994. MR1324339 2.4, 6.1

[9] K.-T. Chen, Integration in free groups, Ann. of Math. (2) 54 (1951), no. 1, 147-162. MR0042414 $1.2,5.1$

[10] K.-T. Chen, Extension of $C^{\infty}$ function algebra by integrals and Malcev completion of $\pi_{1}$, Adv. in Math. 23 (1977), no. 2, 181-210. MR0458461 1.8, 14.1

[11] T. Church, M. Ershov, A. Putman, On finite generation of the Johnson filtrations, arxiv: 1711. Q4779v3, to appear in J. Eur. Math. Soc. (JEMS). 3.1

[12] T. Cochran, Noncommutative knot theory, Algebr. Geom. Topol. 4 (2004), 347-398. MR2077670 $1.2,3.1$

[13] T. Cochran, S. Harvey, Homology and derived series of groups, Geom. Topol. 9 (2005), 2159-2191. MR2209369 1.2, 3.1, 5.2, 5.3

[14] T. Cochran, S. Harvey, Homology and derived p-series of groups, J. London Math. Soc. 78 (2008), 677-692. MR2456898 1.2, 4.1, 4.2

[15] T. Cochran, S. Harvey, Homological stability of series of groups, Pacific J. Math. 246 (2010), nr. 1, 31-47. MR2645878 1.2, 4.1

[16] D.C. Cohen, H.K. Schenck, Chen ranks and resonance, Adv. Math. 285 (2015), 1-27. MR3406494 $1.8,6.10$

[17] D.C. Cohen, A.I. Suciu, Alexander invariants of complex hyperplane arrangements, Trans. Amer. Math. Soc. 351 (1999), no. 10, 4043-4067. MR1475679 2.3, 6.10

[18] R.H. Crowell, Torsion in link modules, J. Math. Mech. 14 (1965), 289-298. MR0174606 2.3 
[19] G. Denham, A.I. Suciu, S. Yuzvinsky, Abelian duality and propagation of resonance, Selecta Math. 23 (2017), no. 4, 2331-2367. MR3703455 8.1, 12.3, 16.1

[20] A. Dimca, R. Hain, S. Papadima, The abelianization of the Johnson kernel, J. Eur. Math. Soc 16 (2014) no. 4, 805-822. MR3191977 1.3, 1.8, 3.1, 6.5, 14.3, 14.4, 14.3

[21] A. Dimca, S. Papadima, Finite Galois covers, cohomology jump loci, formality properties, and multinets, Ann. Sc. Norm. Super. Pisa Cl. Sci. 10 (2011), no. 2, 253-268. MR2856148 14.7, 14.4

[22] A. Dimca, S. Papadima, Arithmetic group symmetry and finiteness properties of Torelli groups, Ann. Math. 177 (2013), no. 2, 395-423. MR3010803 1.8, 3.1, 15.1, 16.2, 16.2, 16.4, 16.8

[23] A. Dimca, S. Papadima, A.I. Suciu, Formality, Alexander invariants, and a question of Serre, unpublished manuscript, arXiv: math. AT/0512480v3. 1.8, 16.2, 16.2

[24] A. Dimca, S. Papadima, A.I. Suciu, Alexander polynomials: Essential variables and multiplicities, Int. Math. Res. Not. IMRN 2008, no. 3, Art. ID rnm119, 36 pp. MR2416998 1.6, 11.2

[25] A. Dimca, S. Papadima, A.I. Suciu, Topology and geometry of cohomology jump loci, Duke Math. Journal 148 (2009), no. 3, 405-457. MR2527322 1.1, 1.6, 1.8, 14.3, 14.3, 14.5, 15.4, 15.3, 16.3, 16.3

[26] W.G. Dwyer, D. Fried, Homology offree abelian covers. I, Bull. London Math. Soc. 19 (1987), no. 4, 350-352. MR0887774 1.6, 12.3, 12.3, 12.4

[27] D. Eisenbud, Commutative algebra, with a view towards algebraic geometry, Grad. Texts in Math., vol. 150, Springer-Verlag, New York, 1995. MR1322960 12.1, 12.2

[28] D. Eisenbud, The geometry of syzygies, a second course in commutative algebra and algebraic geometry, Grad. Texts in Math., vol. 229, Springer-Verlag, New York, 2005. MR2103875 15.2

[29] M. Falk, R. Randell, The lower central series of a fiber-type arrangement, Invent. Math. 82 (1985), no. 1, 77-88. MR808110 1.4, 7.1, 7.2

[30] J. Guaschi, C.M. Pereiro, Lower central and derived series of semi-direct products, and applications to surface braid groups, J. Pure Appl. Algebra 224 (2020), no. 7, 106309, 39 pp. MR4058243 1.4, 7.1, 7.1

[31] R. Hain, Relative weight filtrations on completions of mapping class groups, in: Groups of diffeomorphisms, 309-368, Adv. Stud. Pure Math., vol. 52, Math. Soc. Japan, Tokyo, 2008. MR2509715 $14.3,14.4$

[32] S.L. Harvey, Higher-order polynomial invariants of 3-manifolds giving lower bounds for the Thurston norm, Topology 44 (2005), no. 5, 895-945. MR2153977 1.2, 3.1, 3.1, 3.1

[33] P.J. Hilton, U. Stammbach, A course in homological algebra, Second ed., Graduate Texts in Mathematics, vol. 4, Springer-Verlag, New York, 1997. MR1438546 6.1, 8.1

[34] E. Hironaka, Alexander stratifications of character varieties, Ann. Inst. Fourier (Grenoble) 47 (1997), no. 2, 555-583. MR1450425 1.6, 11.2, 11.2

[35] M. Lackenby, New lower bounds on subgroup growth and homology growth, Proc. Lond. Math. Soc. (3) 98 (2009), no. 2, 271-297. MR2481949 5.3

[36] M. Lackenby, Detecting large groups, J. Algebra 324 (2010), no. 10, 2636-2657. MR2725193 4.1, 4.1, 5.3, 5.3

[37] M. Lazard, Sur les groupes nilpotents et les anneaux de Lie, Ann. Sci. École Norm. Sup. (3) 71 (1954), 101-190. MR0088496 5.1

[38] A. Libgober, On the homology of finite abelian coverings, Topology. Appl. 43 (1992), 157-166. MR1152316 1.6, 12.3

[39] W. Magnus, A. Karrass, D. Solitar, Combinatorial group theory, Second revised edition, Dover, New York, 1976. MR0422434 5.1

[40] M. Markl, S. Papadima, Homotopy Lie algebras and fundamental groups via deformation theory, Ann. Inst. Fourier (Grenoble) 42 (1992), no. 4, 905-935. MR1196099 1.2, 14.1, 14.1, 14.2

[41] W. S. Massey, Completion of link modules, Duke Math. J. 47 (1980), no. 2, 399-420. MR0575904 $1.1,1.2,1.3,2.2,2.3,6.2,6.1,6.2,6.3$ 
[42] G. Massuyeau, Finite-type invariants of 3-manifolds and the dimension subgroup problem, J. Lond. Math. Soc. (2) 75 (2007), no. 3, 791-811. MR2352736 5.2, 5.2, 7.2

[43] D. Matei, A.I. Suciu, Cohomology rings and nilpotent quotients of real and complex arrangements, Arrangements-Tokyo 1998, Adv. Stud. Pure Math., vol. 27, Kinokuniya, Tokyo, 2000, pp. 185-215. MR1796900 16.1, 17.5

[44] D. Matei, A. Suciu, Hall invariants, homology of subgroups, and characteristic varieties, Internat. Math. Res. Notices 2002 (2002), no. 9, 465-503. MR1884468 1.6, 13.12

[45] S. Papadima, A.I. Suciu, Chen Lie algebras, Int. Math. Res. Not. 2004 (2004), no. 21, 1057-1086. MR2037049 1.2, 1.8, 5.1, 14.1, 14.1, 14.2, 14.3, 14.4, 15.1, 15.1, 15.3, 15.4, 15.5

[46] S. Papadima, A.I. Suciu, Algebraic invariants for right-angled Artin groups, Math. Ann. 334 (2006), no. 3, 533-555. MR2207874 14.1, 17.1, 17.8

[47] S. Papadima, A.I. Suciu, Algebraic invariants for Bestvina-Brady groups, J. London Math. Soc. 76 (2007), no. 2, 273-292. MR2363416 1.5, 1.9, 8.5, 8.9, 13.8, 17.8

[48] S. Papadima, A.I. Suciu, Toric complexes and Artin kernels, Adv. Math. 220 (2009), no. 2, 441-477. MR2466422 9.6, 17.8

[49] S. Papadima, A. Suciu, Geometric and algebraic aspects of 1-formality, Bull. Math. Soc. Sci. Math. Roumanie 52 (2009), no. 3, 355-375. MR2554494 1.6, 14.3, 14.4

[50] S. Papadima, A.I. Suciu, Bieri-Neumann-Strebel-Renz invariants and homology jumping loci, Proc. London Math. Soc. 100 (2010), no. 3, 795-834. MR2640291 1.6, 12.3, 12.3, 12.4

[51] S. Papadima, A.I. Suciu, Homological finiteness in the Johnson filtration of the automorphism group of a free group, J. Topol. 5 (2012), no. 4, 909-944. MR3001315 1.8, 15.1

[52] S. Papadima, A.I. Suciu, Jump loci in the equivariant spectral sequence, Math. Res. Lett. 21 (2014), no. 4, 863-883. MR3275650 12.3, 16.1, 16.4, 16.8

[53] S. Papadima, A.I. Suciu, Vanishing resonance and representations of Lie algebras, J. Reine Angew. Math. 706 (2015), 83-101. MR3393364 1.8, 3.3, 15.1, 15.2, 16.4

[54] L. Paris, Residual p properties of mapping class groups and surface groups, Trans. Amer. Math. Soc. 361 (2009), no. 5, 2487-2507. MR2471926 1.4, 5.3, 5.3

[55] D.S. Passman, The algebraic structure of group rings, Pure and Applied Mathematics, WileyInterscience, New York-London-Sydney, 1977. MR0470211 5.1, 5.2, 5.2

[56] D.G. Quillen, On the associated graded ring of a group ring, J. Algebra 10 (1968), 411-418. MR0231919 14.3

[57] D. Quillen, Rational homotopy theory, Ann. of Math. (2) 90 (1969), 205-295. MR0258031 1.8, 14.3, 14.4

[58] P.B. Shalen, P. Wagreich, Growth rates, $\mathbb{Z}_{p}$-homology, and volumes of hyperbolic 3-manifolds, Trans. Amer. Math. Soc. 331 (1992), no. 2, 895-917. MR1156298 5.3

[59] J. Stallings, Homology and central series of groups, J. Algebra 2 (1965), no. 2, 170-181. MR0175956 $1.2,5.2,5.3,8.1,14.3,14.4$

[60] J.R. Stallings, Surfaces in three-manifolds and nonsingular equations in groups, Math. Z. 184 (1983), no. 1, 1-17. MR0711725 1.2, 4.1, 4.1, 4.1

[61] A.I. Suciu, Fundamental groups of line arrangements: enumerative aspects, in: Advances in algebraic geometry motivated by physics (Lowell, MA, 2000), 43-79, Contemp. Math., vol. 276, Amer. Math. Soc., Providence, RI, 2001. MR1837109 1.8, 6.10

[62] A.I. Suciu, Characteristic varieties and Betti numbers of free abelian covers, Int. Math. Res. Notices 2014 (2014), no. 4, 1063-1124. MR3168402 1.6, 12.3, 12.4, 13.1, 13.2

[63] A.I. Suciu, Hyperplane arrangements and Milnor fibrations, Ann. Fac. Sci. Toulouse Math. 23 (2014), no. 2, 417-481. MR3205599 13.10, 14.4, 17.1

[64] A.I. Suciu, On the topology of Milnor fibrations of hyperplane arrangements, Rev. Roumaine Math. Pures Appl. 62 (2017), no. 1, 191-215. MR3626439 1.7, 13.4 
[65] A.I. Suciu, Poincaré duality and resonance varieties, Proc. Roy. Soc. Edinburgh Sect. A 150 (2020), nr. 6, 3001-3027. MR4190099 16.1, 16.1

[66] A.I. Suciu, Lower central series and split extensions, arXiv:2105.14129v1 1.4, 5.2, 5.2, 5.3, 7.1, 7.2, 7.4, 7.5, 7.3, 7.3, 7.7, 7.3

[67] A.I. Suciu, Milnor fibrations of arrangements with trivial algebraic monodromy, draft (2021). 1.7, $1.9,13.4$

[68] A.I. Suciu, H. Wang, Pure virtual braids, resonance, and formality, Math. Z. 286 (2017), no. 3-4, 1495-1524. MR3671586 15.1, 15.4, 15.2, 15.4

[69] A.I. Suciu, H. Wang, Cup products, lower central series, and holonomy Lie algebras, J. Pure Appl. Algebra 223 (2019), no. 8, 3359-3385. MR3926216 5.1, 5.1, 14.1, 14.1, 14.1, 14.2, 14.4, 17.5

[70] A.I. Suciu, H. Wang, Formality properties of finitely generated groups and Lie algebras, Forum Math. 31 (2019), no. 4, 867-905. MR3975666 1.2, 5.1, 14.2, 14.2, 14.3, 14.4, 14.8, 17.2

[71] A.I. Suciu, H. Wang, Chen ranks and resonance varieties of the upper McCool groups, Adv. in Appl. Math. 110 (2019), 197-234. MR3983125 6.10, 15.1

[72] A.I. Suciu, H. Wang, Taylor expansions of groups and filtered-formality, Eur. J. Math. 6 (2020), nr. 3, 1073-1096. MR4151729 6.5, 14.3

[73] A.I. Suciu, Y. Yang, G. Zhao, Homological finiteness of Abelian covers, Ann. Sc. Norm. Super. Pisa Cl. Sci. 14 (2015), no. 1, 101-153. MR3379489 12.3, 12.3, 12.4

Department of Mathematics, Northeastern University, Boston, MA 02115, USA

Email address: a. suciu@northeastern. edu

$U R L$ : web. northeastern. edu/suciu/ 